Universidade de Brasília

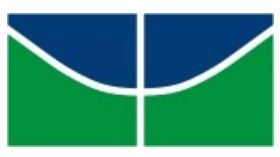

Instituto de Ciências Exatas

Departamento de Matemática

Programa de Mestrado Profissional em PROFMAT

Matemática em Rede Nacional

\title{
Reconstruindo os Determinantes: Uma proposta para o Ensino Médio
}

\section{Hallyson Gomes Monsores}




\title{
Universidade de Brasília
}

\author{
Decanato de Pesquisa e Pós-graduação (DPP)
}

FORMULÁRIO DE DECLARAÇÃO DE CIENCIA DE PROPRIEDADE INTELECTUAL DE MONOGRAFIA/TESE/DISSERTAÇÃO E PRODUTOS DESENVOLVIDOS NA UnB.

(a ser assinado por estudantes e entregue no momento da matricula e na ocasião da defesa do trabalho final)

Eu,

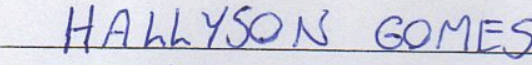

$\mathrm{CPF}$

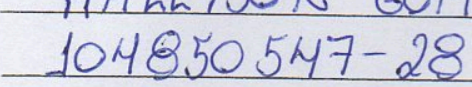

\section{MES}

MONSORES (nome),

Universidade de Brasilia no grau de aluno

(a) regularmente matriculado

(a) na

Mestrado ou Doutorado) em MESTRADO (Residência, Especialização, programa ou curso), considerando MATEMÁTICA (nome do progra ou curo), con direitos e obrigações relativos à propriedade industrial, Lei n 9.609, de 19 de fevereiro de 1998 que dispõe sobre a proteção da propriedade intelectual de programa de computador, sua comercialização no Pais, e dá outras providências, Lei $n^{\circ} 10.973$, de 2 de dezembro de 2004 que dispõe sobre incentivos à inovação e à pesquisa científica e tecnológica no ambiente produtivo e dá outras providências, Lei n 9.456, de 25 de abril de 1997 que institui a Lei de Proteção de Cultivares e dá outras providências, Lei $n^{\circ} 11.788$, de 25 de setembro de 2008 que dispõe sobre o estágio de estudantes, entre outras, bem como qualquer outra Lei que venha substituir ou complementar as já citadas, DECLARO conhecer que os projetos, produtos desenvolvidos, trabalhos publicados e relacionados aos respectivos trabalhos de conclusão final ou outros desenvolvidos por ocasião da estada do estudante nesta Universidade são de propriedade da Universidade de Brasilia, dos respectivos estudante e orientador no âmbito da UnB e quaisquer outros documentos, acordos, termos, cessão de direitos, ou outros assemelhados para esse fim NÃO PODERÃO ser assinados com outra Instituição, Empresa, Agência de Fomento, bolsas, entre outras, sem o prévio conhecimento e concordância da Universidade de Brasilia (UnB), através do Decanato de Pesquisa e Pós-graduação (DPP). A Universidade poderá compartilhar os direitos, produtos, "royalities", patentes etc, desde que previamente acordado conforme a legislação vigente. A presente declaração é assinada formalmente considerando os Art. 297-299 do Código Penal Brasileiro.

$$
\text { Brasilia, } 23 \text { de Tulhe de } 2015
$$

Assinatura do estudante de Pós-graduação: Ylallyson Gomes Nemseres

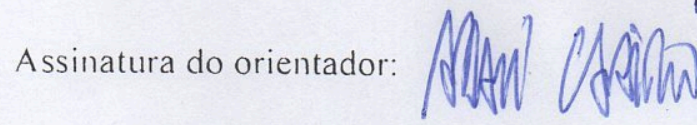




\section{Hallyson Gomes Monsores}

\section{Reconstruindo os Determinantes: Uma proposta para o Ensino Médio}

Trabalho de Conclusão de Curso apresentado ao Departamento de Matemática da Universidade de Brasília, como parte dos requisitos para obtenção do grau de Mestre. Orientador: Profa Dra Aline Pinto

Brasília 
Ficha catalográfica elaborada automaticamente, com os dados fornecidos pelo(a) autor(a)

RECONSTRUINDO OS DETERMINANTES: UMA PROPOSTA PARA O ENSINO MÉDIO / HALLYSON GOMES MONSORES; orientador ALINE PINTO. -- Brasília, 2015.

$100 \mathrm{p}$.

Dissertação (Mestrado - Mestrado Profissional em Matemática) -- Universidade de Brasília, 2015.

1. RECONSTRUÇÃO DOS DETERMINANTES. 2. EVOLUÇÃO HISTÓRICA. 3. EXISTÊNCIA E UNICIDADE DO DETERMINANTE. I. PINTO, ALINE, orient. II. Título. 
Universidade de Brasília

Instituto de Ciências Exatas

Departamento de Matemática

Reconstruindo os Determinantes. Uma proposta para o Ensino Médio por

\section{HALLYSON GOMES MONSORES *}

Dissertação apresentada ao Departamento de Matemática da Universidade de Brasília, como parte dos requisitos do "Programa" de Mestrado Profissional em Matemática em Rede Nacional - PROFMAT, para obtenção do grau de

\section{MESTRE}

Brasília, 23 de julho de 2015.

Comissão Examinadora:

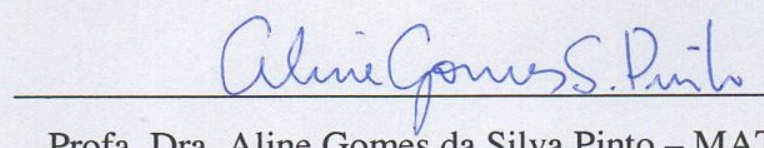

Profa. Dra. Aline Gomes da Silva Pinto - MAT/UnB (Orientadora)

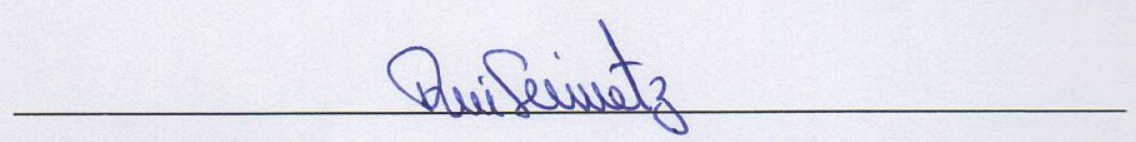

Prof. Dr. Rui Seimetz- MAT/UnB

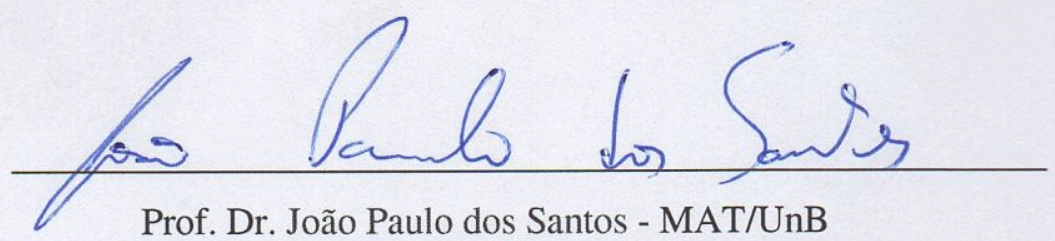

Prof. Dr. João Paulo dos Santos - MAT/UnB

* O autor foi bolsista CAPES durante a elaboração desta dissertação. 
Este trabalho é dedicado à minha esposa, Sirleide Neres Dos Santos que sempre esteve ao meu lado, dando o apoio e compreensão que eu precisava. Sem esta mulher maravilhosa, este trabalho não seria possível nem apresentável. 


\section{Agradecimentos}

Agradeço, primeiramente, a Deus que nós momentos de maior fraqueza, me deu a força necessária para continuar. Dou graças pela minha esposa que esteve ao meu lado em todos os momentos, mesmo naqueles mais difíceis e cansativos. A minha orientadora pela grande dedicação que empenhou para que estas páginas tivessem conteúdo de qualidade e pela paciência que demonstrou comigo nas horas mais turbulentas. A todos os professores do Departamento de Matemática, em especial àqueles que estiveram mais próximos através das aulas ministradas por contribuir não só com conhecimento, mas também com a amizade construída. A todos os alunos da turma Profmat 2013 que unida combateu as dificuldades do curso ajudando uns aos outros sempre que isso se fez necessário, pois sem estes, o caminho teria sido muito mais árduo. Enfim, agradeço a todos que de alguma forma, direta ou indiretamente, fizeram parte da construção desse que agora vos escreve. Agradeço ao CAPES pelo apoio financeiro à este trabalho. 


\section{Resumo}

A palavra 'determinante' e seu significado geralmente são mal compreendidas pelos alunos no Ensino Médio. A natureza abstrata deste termo e as inadequadas técnicas tradicionais de ensino podem explicar esta situação. Depois de esclarecer o significado de 'determinante', esta dissertação visa provar a sua existência. Mais especificamente, nós mostramos que existe a função determinante para matrizes de qualquer ordem $n$. Além disso, apresentamos métodos para facilitar o cálculo dos determinantes nos casos mais complexos. Finalmente, propomos técnicas práticas para resolução de sistemas de equações em contextos escolares.

\section{Palavras-chave}

Determinante, abstração, entendimento. 


\begin{abstract}
The word 'determinant' and its meaning are often poorly understood by school students. The abstract nature of this term and unsuitable traditional teaching techniques may explain this situation. After clarifying the meaning of 'determinant', this dissertation seeks to prove its existence. More specifically, we show that the determinant function exists for matrices of any order $n$. Moreover, we present methods to facilitate the calculation of determinants in more complex cases. Finally, we propose practical techniques for solving systems of equation in school contexts.
\end{abstract}

\title{
Keywords
}

Determinant, abstraction, understanding. 


\section{Lista de Figuras}

2.1 retas concorrentes . . . . . . . . . . . . . . . . 28

2.2 retas coincidentes . . . . . . . . . . . . . . . . . . . 29

2.3 retas paralelas . . . . . . . . . . . . . . . . 30

2.4 Planos cuja intersecção é um ponto . . . . . . . . . . . . . . . 43

2.5 Planos cuja intersecção é uma reta . . . . . . . . . . . . . 43

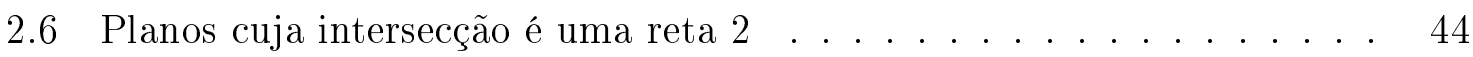

2.7 Planos cuja intersecção é um plano . . . . . . . . . . . . . . . . . . 44

2.8 Planos sem intersecção tripla . . . . . . . . . . . . . . . . . . 44

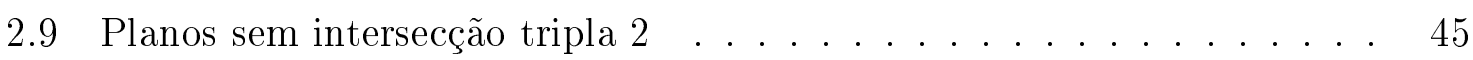

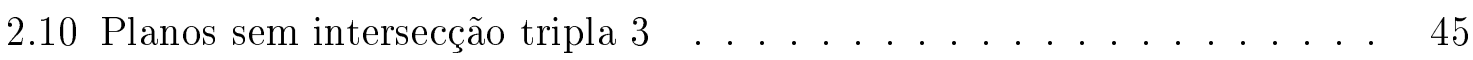




\section{Sumário}

1 Origem e evolução histórica $\quad 15$

2 Reconstruindo o Determinante 20

2.1 Determinante de ordem 2 . . . . . . . . . . . . . 20

2.1 .1 Casos do Sistema . . . . . . . . . . . . . 25

2.1.2 Indexando os Elementos da Matriz . . . . . . . . . . . . 26

2.1.3 Analogia Geométrica dos Casos do Sistema . . . . . . . . . . 27

2.2 Determinante de ordem $3 \ldots \ldots \ldots$. . . . . . . . . . . 31

2.2.1 Casos do Sistema . . . . . . . . . . . . . 39

2.2.2 Indexando os Elementos da Matriz . . . . . . . . . . . . 39

2.2.3 Regra de Sarrus . . . . . . . . . . . . . . . 40

2.2.4 Analogia Geométrica dos Casos do Sistema . . . . . . . . . . 42

3 Matrizes $\quad 46$

3.1 Tipos de Matrizes . . . . . . . . . . . . . . . . 46

3.2 Operações com Matrizes . . . . . . . . . . . . . . . . 49

3.2 .1 Adição de matrizes . . . . . . . . . . . . . . . . 49

3.2.2 Multiplicação de um escalar por uma matriz . . . . . . . . . . 51

3.2.3 Multiplicação de matrizes . . . . . . . . . . . . 52

4 Fundamentação Teórica do Determinante $\mathbf{5 5}$

4.1 Funções Determinantes . . . . . . . . . . . . . . 56

4.2 Unicidade da Função Determinante . . . . . . . . . . . . . . 65

4.3 Propriedades dos Determinantes . . . . . . . . . . . . 71

4.4 Abaixamento de ordem de um determinante (Regra de Chió) . . . . . 81

4.5 Regra de Crammer .................. 83 
5 Atividades Propostas $\quad \mathbf{8 6}$

5.1 Atividades sobre matrizes . . . . . . . . . . . . 86

5.2 Atividades sobre Determinantes . . . . . . . . . . . . . 90 


\section{Introdução}

De forma geral, ao passarmos pelo ensino médio, nos deparamos com as noções de matrizes e determinantes de maneira mecanizada e sem a preocupação de uma contextualização histórica do surgimento do assunto bem como uma explicação prática dos objetivos para os quais foram criados.

Geralmente, após uma breve e não muito eficiente conceituação algébrica, as matrizes são expostas ao aluno como tabelas de valores agrupadas em linhas e colunas e os determinantes são mostrados como valores reais associados a matrizes quadradas e obtidos por um método algoritmizado. Quase sempre, o aluno se depara com dúvidas que não são respondidas no ensino médio, o que acaba por mecanizar o ensino e na maioria das vezes desestimula o interesse dos alunos a cerca do objeto estudado.

Perguntas essenciais ao entendimento do assunto como "O que é o determinante?", "O que é uma matriz?", "Para que serve o determinante?", "Por que só matrizes quadradas possuem determinante?", entre outras, não são respondidas no ensino médio o que faz com que o aluno absorva apenas a algoritmização mas não entenda o sentido e o procedimento utilizado, em outras palavras, os alunos replicam o procedimento mecanizado sem saber o que estão fazendo nem para que o fazem.

Neste trabalho, abordaremos o assunto "Determinantes" trazendo a luz sua origem gradual na história da matemática mostrando assim de forma mais clara o que é o determinante, como ele surgiu, como evoluiu até os dias modernos, quais eram os objetivos iniciais ao se estudar esse assunto e em que áreas da ciência matemática ele é utilizado hoje.

Por meio do processo de reconstrução da resolução de sistemas de equações lineares mostraremos de forma algébrica generalizada o que é o determinante nos casos de $2^{\mathrm{a}}$ e $3^{\mathrm{a}}$ ordem, como ele foi desenvolvido e como esses determinantes são importantes na facilitação da resolução desses sistemas. Trataremos esse processo como uma proposta alternativa de introdução ao estudo dos determinantes em detrimento ao pro- 
cesso tradicional de ensino aplicado. O processo que utilizaremos na reconstrução dos determinantes de $2^{\mathrm{a}}$ e $3^{\mathrm{a}}$ ordem, apesar de totalmente algébrico é bem básico, consistindo somente em multiplicação de equações e eliminação de incógnitas, logo pode ser utilizado em sala no ensino médio para introduzir o estudo dos determinantes, pois para entendê-lo basta que o aluno esteja familiarizado com a resolução de sistemas lineares pelo método da eliminação de incógnitas.

Cabe salientar que para determinantes de ordem maior que 3, o sistema de reconstrução algébrica pela generalização da solução de equações lineares começa a ficar muito trabalhoso e improdutivo, utilizando letras demais para representar os coeficientes e necessitando de muitas operações algébricas para concluí-lo. Como a maioria dos sistemas de equações estudados no ensino médio são de $2^{\mathrm{a}}$ ou de $3^{\mathrm{a}}$ ordem, torna-se desnecessário generalizar o determinante para as demais, porém com o "Teorema de Laplace" que estudaremos também neste trabalho, torna-se possível calcular o determinante aplicado sobre matrizes de qualquer ordem.

Passando a um nível mais elevado do estudo, enunciaremos e provaremos a existência de uma função denominada como "Função Determinante" que é associada a matrizes de ordem $n$, em seguida nos dedicaremos a mostrar que essa função é única, ou seja que só existe uma função determinante aplicada sobre matrizes de ordem $n$ e por último abordaremos diversas propriedades e Teoremas que facilitam o cálculo e o trabalho com determinantes de qualquer ordem. 


\section{Capítulo 1}

\section{Origem e evolução histórica}

Segundo Boyer ([3], pp. 133-135) e Iezzi e Hazzan ([1], pp. 125-126), apesar de algumas divergências entre os historiadores da matemática, em um aspecto eles convergem, o estudo dos determinantes teve sua origem mais remota iniciada pela necessidade de resolução dos sistemas de equações lineares. Foram encontrados registros de tabuletas em argila babilônicas datadas de aproximadamente 300 anos a.c. onde encontramos os primeiros registros conhecidos de sistemas de equações lineares de duas equações e duas incógnitas sendo utilizados para resolver problemas matemáticos.

Ainda na antiguidade os orientais começaram a estudar esse tipo de sistema de equações. O chineses, especialistas em diagramas e quadrados mágicos, representavam sistemas de equações lineares através de seus coeficientes escritos em barras de bambu sobre os quadrados de um tabuleiro.

Aproximadamente no século II a.c. foi publicado na China, o livro "Os nove capítulos sobre a arte matemática" que propunha cerca de 246 problemas sobre mensuração de terras, agricultura, sociedades, engenharia, impostos, cálculos, solução de equações e propriedades dos triângulos retângulos a serem resolvidos por meio de sistemas de equações lineares. De forma quase que algoritmizada o livro resolvia os sistemas por eliminação, anulando os coeficientes das incógnitas através de operações elementares muito semelhantes ao que fazemos hoje em dia ao escalonar um sistema linear pelo processo de Gauss. Um dos primeiros problemas deste livro e talvez aquele que tenha ganhado maior repercussão continha o seguinte enunciado:

"Havia três tipos de milho: Três pacotes do primeiro tipo, dois do segundo e um do terceiro, totalizando 39 unidades de milho. Dois pacotes do primeiro, três pacotes 
do segundo e um pacote do terceiro, totalizando 34 unidades de milho e, um pacote do primeiro, dois do segundo e três do terceiro, somando 26 unidades de milho. Sabendo que os pacotes do mesmo tipo de milho, levam a mesma quantidade de unidades, quantas unidades de milho contem um pacote de cada tipo?"

A resolução dada pelo livro agrupa os coeficientes das incógnitas (pacotes de cada tipo de milho) organizados em uma tabela muito parecida com o que seria hoje uma matriz de ordem $4 \times 3$, como podemos visualizar abaixo. Em seguida são feitas diversas operações elementares que objetivam zerar os coeficientes um a um e assim chegar a uma equação mais simples com uma única incógnita. Após encontrar a solução para essa incógnita o sistema vai sendo resolvido substituindo o valor encontrado, encontrando o valor da próxima incógnita e assim sucessivamente. Obviamente esse procedimento hoje é o que mais se aproxima do escalonamento pelo método de Gauss.

$$
\left(\begin{array}{ccc}
1 & 2 & 3 \\
2 & 3 & 2 \\
3 & 1 & 1 \\
26 & 34 & 39
\end{array}\right) \Rightarrow\left(\begin{array}{ccc}
0 & 0 & 3 \\
0 & 5 & 2 \\
36 & 1 & 1 \\
99 & 24 & 39
\end{array}\right)
$$

Apesar de o inicio dos estudos acerca desse assunto datar de antes de cristo, este ficou inerte e sem evolução material e somente no final do século XVII que a ideia de determinante começou a tomar corpo, mais especificamente foi no trabalho do matemático japonês Seki Takakazu Kowa (1642-1708) em 1683 que o método chinês foi aprimorado e foi padronizado uma forma de resolução do sistema de equações lineares, porém apenas para o caso de duas equações e duas incógnitas. Essa algoritmização do método chinês usava os coeficientes das incógnitas das equações do sistema linear encontrando escalares que solucionavam este sistema. Hoje conhecemos esses escalares como determinantes das matrizes obtidas com os coeficientes das equações e também com os termos independentes.

Segundo Boyer ([3], pp. 279-280), foi Gottfried Wilhelm Leibniz (1649-1716) o primeiro matemático a fazer referência ao estudo dos determinantes no ocidente cerca de 10 anos depois das publicações de Seki Kowa no Oriente. Apesar da maior parte da contribuição de Leibniz para a matemática versar sobre o cálculo, os estudos de Leibniz também foram direcionados no intuito de algoritmizar a resolução de sistemas lineares de três equações e duas incógnitas porém utilizando não uma matriz de ordem 
$2 \times 2$, mas uma matriz de ordem $3 \times 3$ formada pelos coeficientes das equações e pelos termos independentes. O processo de Leibniz foi o mais próximo do que seria hoje o estudo dos determinantes de matrizes de ordem $3 \times 3$. Foi também Leibniz o primeiro matemático a inserir índices para os coeficientes, fato comprovado em cartas datadas de 1693, onde Leibniz conta a Guillaume François L'Hospital (1661-1704) que ocasionalmente utilizava números indicando linhas e colunas para indexar uma coleção de equações simultâneas. Nas figuras 2 e 3 podemos visualizar uma matriz de ordem $3 \times 3$ genericamente mostrada por Leibniz e observamos que ela se aproxima muito da linguagem utilizada hoje nos livros didáticos.

$$
\begin{aligned}
& \left(\begin{array}{ccc}
1_{1} & 1_{2} & 1_{3} \\
2_{1} & 2_{2} & 2_{3} \\
3_{1} & 3_{2} & 3_{3}
\end{array}\right)_{\text {Fig2: Matriz representada por Leibniz }} \\
& \left(\begin{array}{lll}
a_{11} & a_{12} & a_{13} \\
a_{21} & a_{22} & a_{23} \\
a_{31} & a_{32} & a_{33}
\end{array}\right)_{\text {Fig3: Matriz representada atualmente }}
\end{aligned}
$$

Segundo Boyer ([3], pp. 297-298), em 1729, o matemático escocês Colin Maclaurin (1698-1746) foi o primeiro a generalizar o conceito de determinante na solução de sistemas lineares de $n$ equações com $n$ incógnitas, porém seus estudos só foram publicados em 1748, dois anos após sua morte, no trabalho intitulado "Treatise of algebra". Mesmo tendo sido estudada por Maclaurin, o algoritmo de resolução só ganhou realmente notoriedade através dos estudos do suíço Gabriel Cramer (1704-1752). O matemático suíço chegou a praticamente os mesmo resultados de Maclaurin em sua obra "Introdução e Análise das curvas Algébricas" datada de 1750, porém cabe salientar que o objetivo dos estudos de Cramer era focado em determinar os coeficientes das cônicas e seu desenvolvimento do estudo dos determinantes foi apenas um meio para atingir esse objetivo. De qualquer jeito a resolução de sistemas lineares de $n$ equações e $n$ incógnitas por meio do uso de determinantes é até hoje conhecida como "Regra de Cramer". Acredita-se que a resolução de sistemas lineares de $n$ equações e $n$ incógnitas pelo método de Crammer tenha ganhado mais notoriedade que a apresentada por Maclaurin devido a diversos 
motivos, entre eles a superioridade da notação utilizada por Crammer em detrimento a de Maclaurin e também ao declínio momentâneo que passava a comunidade científica da Inglaterra perante a comunidade Européia.

Segundo Boyer ([3], pp. 324-325), Joseph Louis Lagrange (1736-1813) foi o primeiro a utilizar o estudo do determinante em uma aplicação geométrica direta, quando em 1775, publicou seu artigo intitulado "Solutions analytiques de quelques sur les pyramides triangulaires" onde aplicava o estudo dos determinantes para generalizar o cálculo da área de um triângulo qualquer e do volume de um tetraedro qualquer. As ideias de Lagrange abriram um precedente gigantesco pois a partir dai começou-se a perceber como a álgebra aplicada poderia ter valor significativo para aplicações geométricas. Percebemos essa importância de forma velada em uma expressão do próprio Lagrange quando escreveu, "parece-me que as soluções que vou apresentar serão de interesse para os geômetras tanto pelos métodos quanto pelos resultados".

Segundo Iezzi e Hazzan ([1], pp. 125-126), em 1764, o matemático francês Étienne Bézout (1730-1783) criou um processo de estabelecimento dos sinais dos termos de um determinante, mas foi somente em 1771 que Alexandre Theóphile Vandermonde (17351796), também francês, estudou de forma independente de sistemas lineares a teoria dos determinantes, estes foram pela primeira vez estudados com maior importância em si mesmos e não apenas como meio para outros estudos. Segundo Boyer ([3], pp. 320-321), em 1779, em seu trabalho intitulado "Theorie generale des equations algébriques", Bezout ficou ainda mais conhecido por estudar o determinante para um tópico denominado eliminação algébrica, a partir desse estudo ele deu regras artificiais semelhantes as de Crammer para resolver um sistema de $n$ equações simultâneas e $n$ incógnitas em que se deseja achar a condição entre os coeficientes necessária para que as equações possuam uma solução comum.

Segundo Iezzi e Hazzan ([1], pp. 125-126), em 1772, Pierre Simon Laplace (17491827), em seu artigo "Pesquisas sobre o cálculo integral e o sistema do mundo" sintetizou um método que permite o cálculo do determinante de qualquer matriz quadrada através de determinantes menores de uma fila qualquer escolhida na matriz e seus complementos algébricos. Até hoje esse Teorema é globalmente conhecido e utilizado em cálculos de determinantes como Teorema de Laplace e possui importância tão significativa que alguns autores dizem ser o mais importante Teorema no estudo dos determinantes.

Segundo Boyer ([3], pp. 353-359), em 1812, Augustin-Louis Cauchy (1789-1857) reuniu tudo que fora feito até o momento sobre o assunto de determinantes em um único artigo de oitenta e quatro páginas apresentado a Academia de Ciências, além de ter 
simplificado e melhorado varias notações, Cauchy foi o primeiro matemático a atribuir a nomenclatura "Determinante" ao estudo da algoritimização dos coeficientes de $n$ equações com $n$ incógnitas para a resolução genérica de um sistema linear. Nascia então o nome "DETERMINANTE" que é o núcleo fundamental desse trabalho. Embora fosse num contexto completamente diferente, Cauchy derivou a nomenclatura "determinante" de um trabalho de Gauss. Cauchy também descreveu o estudo dos determinantes para resolução de sistemas como uma classe de funções simétricas alternadas o que não traduz bem as referências modernas do assunto. Em um artigo datado de 1815 sobre a propagação de ondas, Cauchy extrapolou o uso específico na álgebra e aplicou o estudo dos determinantes a dois problemas, um de geometria e um de física onde utilizou o determinante para calcular o volume de um paralelepípedo e também a algumas derivadas parciais associadas a propagação de ondas. Cauchy ainda introduziu a noção sobre a multiplicação de determinantes e suas consequências.

Segundo Boyer ([3], pp. 361-364), após Cauchy, Carl Gustav Jacobi (1804-1851) foi o matemático que mais contribuiu para o aperfeiçoamento do estudo dos determinantes e suas notações. Podemos afirmar que grande parte da notação moderna que utilizamos hoje para tratar o assunto foi atribuída por Jacobi que era conhecido como o grande algorista da época. Jacobi deu grande importância também a um estudo específico dentro dos determinantes denominado como determinantes funcionais. Em 1841, ele publicou um longo artigo "De determinantibus functionalibus" especificamente dedicado ao determinante Jacobiano e em suas demonstrações mostrou uma forma prática de utilizar os determinantes funcionais para determinar se um conjunto de equações é ou não independente. 


\section{Capítulo 2}

\section{Reconstruindo o Determinante}

Este capítulo constitui uma proposta didática para o ensino dos determinantes no Ensino Médio com uma abordagem diferente da tradicionalmente utilizada.

Iremos obter, após uma manipulação algébrica de equações, a expressão que traduz o significado do determinante pela resolução de sistemas lineares, ou seja, para os casos de matrizes de $2^{\mathrm{a}}$ e de $3^{\mathrm{a}}$ ordem, iremos deduzir a expressão do determinante associado e não simplesmente defini-la como geralmente é proposto no sistema tradicional de ensino que pudemos constatar nos livros didáticos [4], [5], [6], [7] e [8] utilizados nas escolas públicas do DF.

\section{$2.1 \quad$ Determinante de ordem 2}

Sendo $\mathbb{R}$ o conjunto dos números reais, para obter o determinante das matrizes de $2^{\mathrm{a}}$ ordem, devemos algoritmizar a resolução de um sistema de duas equações e duas incógnitas sobre $\mathbb{R}$, de forma generalizada a fim de chegarmos a expressão determinante de qualquer matriz de ordem 2.

Vamos considerar o seguinte sistema de equações:

$$
\left\{\begin{array}{c}
I: a x+b y=f \\
I I: c x+d y=g
\end{array}\right.
$$


onde $x$ e $y$ representam as incógnitas do sistema, $a$ e $c$ representam os coeficientes de $x, b$ e $d$ representam os coeficientes de $y$ e $f$ e $g$ representam os termos independentes do sistema, todos eles elementos de $\mathbb{R}$, tentaremos algoritmizar a resolução por meio do processo de eliminação de incógnitas.

Sem perda de generalidade vamos eliminar a incógnita $y$ e para isso multiplicaremos toda a equação $I I$ por $\frac{-b}{d}$. De fato, para realizarmos essa operação devemos ter $d \neq 0$. Porém se $d=0, x$ terá um valor bem definido, a saber, $x=\frac{g}{c}$ e dessa forma, podemos substituir o valor de $x$ na segunda equação e calcular o valor de $y$. Cabe salientar que $c$ e $d$ não podem ser ao mesmo tempo iguais a zero, pois se forem, teremos uma equação do tipo $0 x+0 y=g$. Se $g=0$ o sistema terá apenas uma equação, o que não é o objeto do estudo; e se $g \neq 0$ o sistema não terá solução.

Supondo então $d \neq 0$, e multiplicando a equação II por $\frac{-b}{d}$, obtemos a equação

$$
I I_{b}: \frac{-b c}{d} x-b y=\frac{-b g}{d} .
$$

Somando agora a equação $I$ com a equação $I I_{b}$, a incógnita $y$ é eliminada e a equação resultante pode ser desenvolvida da seguinte forma:

$$
x\left(a-\frac{b c}{d}\right)=f-\frac{b g}{d} \Rightarrow x\left(\frac{a d-b c}{d}\right)=\frac{f d-b g}{d} \Rightarrow x(a d-b c)=f d-b g .
$$

Com isso, podemos expressar genericamente o valor da incógnita $x$ através da combinação dos coeficientes e dos termos independentes do sistema por meio da expressão

$$
x(a d-b c)=(f d-b g)
$$

Analisando a expressão, concluímos que $x$ só terá solução caso $a d-b c \neq 0$. Essa análise mostra o que já haviamos mencionado anteriormente sobre a impossibilidade de $c$ e $d$ serem ao mesmo tempo nulos.

Da mesma forma que manipulamos as equações para eliminar a incógnita $y$ e encontrar a solução para $x$, podemos repetir o processo, porém dessa vez manipulamos as equações a fim de eliminarmos a incógnita $x$ e com isso encontrar a solução para $y$. 
Para isso iniciamos multiplicando a Equação $I I$ por $\frac{-a}{c}$. De fato, para realizarmos essa operação devemos supor que $c \neq 0$, analogamente ao que fizemos na primeira manipulação algébrica.

Supondo então $c \neq 0$, e multiplicando a equação II por $\frac{-a}{c}$, obtemos a equação

$$
I I_{c}:-a x-\frac{a d}{c} y=\frac{-a g}{c} .
$$

Somando agora a equação $I$ com a equação $I I_{c}$, a incógnita x é eliminada e a equação resultante pode ser desenvolvida da seguinte forma:

$$
y\left(b-\frac{a d}{c}\right)=f-\frac{a g}{c} \Rightarrow y\left(\frac{b c-a d}{c}\right)=\frac{f c-a g}{c} \Rightarrow y(b c-a d)=f c-a g .
$$

Com isso, podemos expressar genericamente o valor da incógnita $y$ através da combinação dos coeficientes e dos termos independentes do sistema por meio da expressão

$$
y(a d-b c)=(a g-f c)
$$

Analogamente ao estudo que fizemos para a equação que determina a incógnita $x$, percebemos que $y$ só terá solução caso $a d-b c \neq 0$.

A partir disso, e supondo $a d-b c \neq 0$, podemos generalizar a resolução do sistema de equações nas incógnitas $x$ e $y$ como uma combinação de coeficientes onde

$$
x(a d-b c)=(f d-b g) ; y(a d-b c)=(a g-f c)
$$

Observe que a expressão que faz o papel de coeficiente de $x$ e de $y$ nas duas equações é a mesma, e por esse motivo chamaremos esta expressão de determinante de ordem 2. Usaremos algumas vezes a expressão Det para nos referirmos ao valor determinante. Dessa forma temos

$$
\text { Det }=(a d-b c)
$$


Se agruparmos os coeficientes das incógnitas $x$ e $y$ respectivamente em uma matriz, observaremos que o Det é dado pela diferença entre o produto dos coeficientes que estão alocados nas duas diagonais da matriz. Mais a frente, trataremos essas diagonais por diagonal principal e diagonal secundária e definiremos seus elementos de forma indexada.

Utilizando a definição e notação de matriz e de determinante abordada no capitulo 3, vamos agrupar os coeficientes do sistema linear a fim de facilitar o cálculo do determinante.

Assim, na matriz $\left(\begin{array}{cc}a & b \\ c & d\end{array}\right)$, podemos afirmar que o determinante dessa matriz é dado por Det $=\left|\begin{array}{ll}a & b \\ c & d\end{array}\right|=a d-b c$.

Observamos ainda que para determinar o valor da incógnita $x$, não basta apenas o valor determinante Det, precisaremos também do numerador da fração que é dado pela expressão $f d-b g$. A este valor daremos o nome de determinante de $x$ e usaremos a expressão

$$
\operatorname{Det}_{x}=f d-b g
$$

De forma análoga, utilizaremos os coeficientes para formar uma matriz, porém trocaremos os coeficientes da incógnita $x$ pelos termos independentes do sistema, e assim podemos escrever que o determinante de $x$ é o Det da matriz obtida com os termos independentes e os coeficientes de $y$.

$$
\begin{aligned}
& \text { Assim, na matriz }\left(\begin{array}{ll}
f & b \\
g & d
\end{array}\right) \text {, podemos afirmar que o determinante dessa matriz é } \\
& \text { dado por } \text { Det }_{x}=\left|\begin{array}{cc}
f & b \\
g & d
\end{array}\right|=f d-b g .
\end{aligned}
$$

Com o mesmo tipo de observação, podemos perceber que para determinar o valor da incógnita $y$, precisaremos do numerador que é dado pela expressão $a g-f c$. A este 
valor daremos o nome de determinante de $y$ e usaremos a expressão

$$
\operatorname{Det}_{y}=a g-f c .
$$

De forma análoga, utilizaremos os coeficientes para formar uma matriz, porém trocaremos os coeficientes da incógnita $y$ pelos termos independentes do sistema, e assim podemos escrever que o determinante de $y$ é o Det da matriz obtida com os termos independentes e os coeficientes de $y$.

Assim, na matriz $\left(\begin{array}{cc}a & f \\ c & g\end{array}\right)$, podemos afirmar que o determinante dessa matriz é dado por $\operatorname{Det}_{y}=\left|\begin{array}{cc}a & c \\ f & g\end{array}\right|=a g-f c$.

Chegando a esse ponto do estudo podemos generalizar a solução do sistema de duas equações e duas incógnitas $x$ e $y$ da seguinte forma:

Dado o sistema linear de duas equações e duas incógnitas

$$
\left\{\begin{array}{l}
a x+b y=f \\
c x+d y=g
\end{array}\right.
$$

as soluções do sistema podem ser genericamente expressas pelas relações

$$
x(\operatorname{Det})=\operatorname{Det}_{x} ; y(\operatorname{Det})=\operatorname{Det}_{y}
$$

onde:

Det é o determinante da matriz $\left(\begin{array}{cc}a & b \\ c & d\end{array}\right)$ formada pelos coeficientes das incógnitas $x$ e $y$ respectivamente , Det $=\left|\begin{array}{cc}a & b \\ c & d\end{array}\right|=a d-b c$;

Det $_{x}$ é o determinante da matriz $\left(\begin{array}{ll}f & b \\ g & d\end{array}\right)$ formada pelos termos independentes e 
pelos coeficientes da incógnita $y$ e, Det $_{x}=\left|\begin{array}{cc}f & b \\ g & d\end{array}\right|=f d-b g$;

Det $_{y}$ é o determinante da matriz $\left(\begin{array}{cc}a & f \\ c & g\end{array}\right)$ formada pelos coeficientes da incógnita $x$ e pelos termos independeste do sistema e, $\operatorname{Det}_{y}=\left|\begin{array}{ll}a & f \\ c & g\end{array}\right|=a g-f c$.

Cabe salientar que como $x$ e $y$ são determinados por uma razão, esta pode assumir casos diferentes os quais abordaremos a seguir.

\subsubsection{Casos do Sistema}

$\mathbf{1}^{\mathbf{O}} \mathbf{C A S O}-($ Det $\neq 0)$

Sempre que o Det $\neq 0$, a expressão que determina a incógnita $x$ poderá ser solucionada, logo o sistema terá solução possível e unicamente determinada pelo algorítimo de resolução anteriormente demonstrado.

$2^{\mathbf{o}} \mathbf{C A S O}-\left(\operatorname{Det}=0, \operatorname{Det}_{x}=0\right.$ e $\left.\operatorname{Det}_{y}=0\right)$

Sempre que todos os determinantes do sistema forem nulos, as expressões que determinam $x$ e $y$ serão representadas como $0 x=0$ e $0 y=0$ e tais expressões podem ter infinitas soluções. Logo o sistema será indeterminado e terá infinitos pares de solução $(x, y)$.

$3^{\text {o }} \mathbf{C A S O}-\left(\right.$ Det $=0, \operatorname{Det}_{x} \neq 0$ ou $\left.\operatorname{Det}_{y} \neq 0\right)$

Sempre que o determinante for nulo e que os determinantes de $x$ ou de $y$ forem diferentes de zero, as expressões que determinam as incógnitas $x$ ou $y$ serão representadas por $0 x=\operatorname{Det}_{x}$ ou $0 y=\operatorname{Det}_{y}$. Tais expressões não apresentam solução, logo o sistema será impossível. 


\subsubsection{Indexando os Elementos da Matriz}

A fim de definir algumas propriedades da matriz, surgiu a necessidade de generalizar seus elementos de forma mais criteriosa. Para isso foi criado uma forma de indexar os elementos da matriz onde cada elemento recebe uma letra representativa do elemento e um índice que representa a linha e a coluna onde o elemento se encontra na matriz.

Assim, quando indexamos a matriz de ordem 2 que foi utilizada para resolver o sistema de duas equações e duas incógnitas temos

$$
\left(\begin{array}{ll}
a & b \\
c & d
\end{array}\right)=\left(\begin{array}{ll}
a_{11} & a_{12} \\
a_{21} & a_{22}
\end{array}\right)
$$

Podemos perceber que cada elemento $a_{i j}$ da matriz representa o elemento alocado na linha $i$ e na coluna $j$. Dessa forma pode-se estabelecer dois conceitos importantes dentro do estudo das matrizes.

O primeiro estabelece a diagonal principal de qualquer matriz que é o conjunto de elementos $a_{i j}$ desta matriz onde $i=j$. Assim na matriz de ordem 2, a diagonal principal é dada por

$$
D_{p}=\left\{a_{11}, a_{22}\right\}
$$

O segundo estabelece a diagonal secundária de qualquer matriz que é o conjunto de elementos $a_{i j}$ desta matriz onde $i+j$ é igual a ordem da matriz mais um. Assim na matriz de ordem 2, a diagonal secundária é dada por

$$
D_{s}=\left\{a_{12}, a_{21}\right\}
$$

Após estabelecidos os conceitos de diagonais da matriz, podemos reescrever o determinante dessa matriz como

$$
\operatorname{Det}=\left|\begin{array}{ll}
a_{11} & a_{12} \\
a_{21} & a_{22}
\end{array}\right|=a_{11} a_{22}-a_{21} a_{12}
$$


Outra forma de escrever o determinante dessa matriz é defini-lo como a diferença entre o produto dos elementos da diagonal principal e o produto dos elementos da diagonal secundária.

\subsubsection{Analogia Geométrica dos Casos do Sistema}

Cada um dos casos do sistema de duas equações e duas incógnitas que foram mostrados nesta subseção pode ser representado geometricamente por posições relativas entre duas retas no plano. Esta analogia atribui significado adicional ao entendimento dos casos do sistema e pode ter papel importante no trabalho com alunos do Ensino Médio caso sejam abordadas da forma e no momento corretos.

Como verificamos nas referências [4], [5], [6], [7] e [8], a maioria das escolas, em especial as do ensino público, não associam a resolução de sistemas de equações lineares com as retas no plano, tratando estes dois assuntos de forma totalmente separada e sem nenhum tipo de analogia posterior. Parece óbvio, mas muitos não percebem o grande potencial de ensino e correlação de conteúdos que possui este assunto, podendo trabalhar conjuntamente diversos tópicos do ensino matemático como estudo das retas no plano, funções, sistema de equações do primeiro grau, matrizes e determinantes, dentre outros.

Nos parágrafos abaixo, abordaremos de forma superficial uma analogia geométrica entre retas no plano a cada um dos casos que o sistema de duas equações e duas incógnitas pode assumir, salientando que essa abordagem pode ser bem mais explorada pelo professor em sala de aula caso constate que a turma tem os pré requisitos necessários para tal. 
$\mathbf{1}^{\circ}$ CASO $-(D e t \neq 0)$

Como ja vimos anteriormente, neste caso a solução do sistema é única e determinada, ou em outras palavras, só existe um par ordenado $(x, y)$ que soluciona o sistema de equações.

Ao fazermos uma analogia com o estudo das retas, representamos cada uma das equações do sistema como uma equação da reta no plano, e com isso as soluções do sistema serão pares ordenados $(x, y)$ que estejam ao mesmo tempo nas duas retas. Como neste caso a solução é única, só pode existir um ponto no plano que esteja contido ao mesmo tempo nas duas retas.

Estudando as três posições relativas possíveis entre duas retas no plano, percebemos que a única que atende a este caso específico é a de retas concorrentes em um ponto, e este ponto será também a solução do sistema como podemos observar na figura 2.1

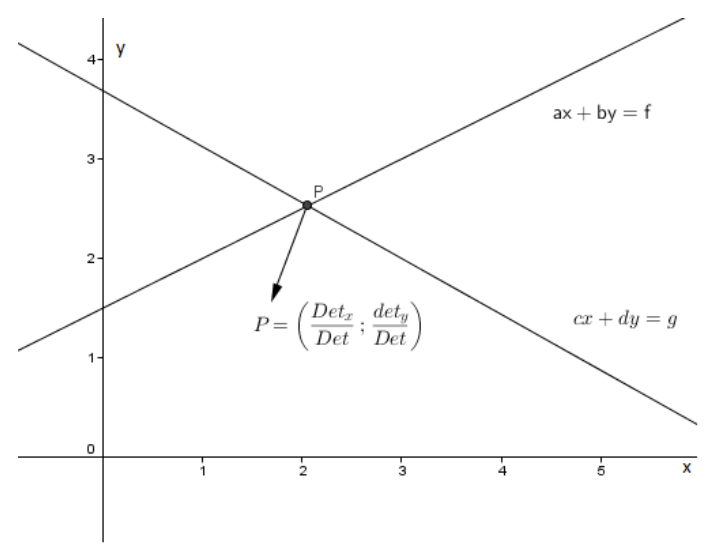

Figura 2.1: retas concorrentes 
$2^{\text {o CASO }}-\left(\right.$ Det $=0$, Det $_{x}=0$ e Det $\left._{y}=0\right)$

Como ja vimos anteriormente, neste caso, a solução do sistema é indeterminada, ou em outras palavras, existem infinitos pares ordenados $(x, y)$ que solucionam esse sistema.

Ao fazermos uma analogia com o estudo das retas, percebemos que esses pares ordenados que solucionam o sistema devem estar contidos nas duas retas, e para que duas retas contenham mais de um par ordenado em comum, sabemos que essas retas devem ser coincidentes, ou seja, as duas equações do sistema, mesmo que com argumentos diferentes, representam a mesma reta no plano como podemos observar na figura 2.2

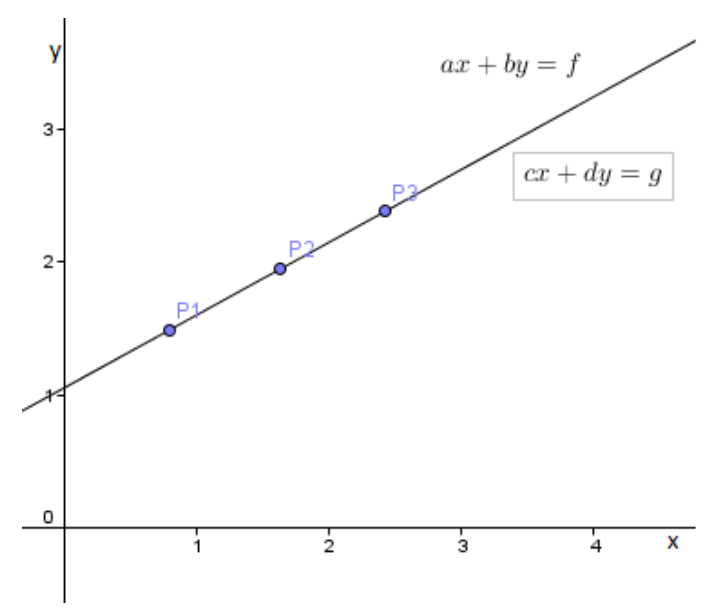

Figura 2.2: retas coincidentes 
Podemos perceber que qualquer ponto que esteja na reta, como por exemplo $P_{1}, P_{2}$, $P_{3}$, são soluções desse sistema, e como existem infinitos pontos em uma reta, existem também infinitas soluções para este sistema de equações.

$3^{\mathbf{o}} \mathbf{C A S O}-\left(\operatorname{Det}=0, \operatorname{Det}_{x} \neq 0 \operatorname{Det}_{y} \neq 0\right)$

Como ja vimos anteriormente, neste caso, a solução do sistema é impossível, ou em outras palavras, não existe nenhum par ordenados $(x, y)$ que solucione esse sistema.

Ao fazermos uma analogia com o estudo das retas, percebemos que neste caso, para não existir nenhum par ordenado que esteja ao mesmo tempo nas duas retas, é necessário que essas retas não se intersectem em nenhum ponto, ou seja, essas retas devem ser paralelas como observaremos na figura abaixo.

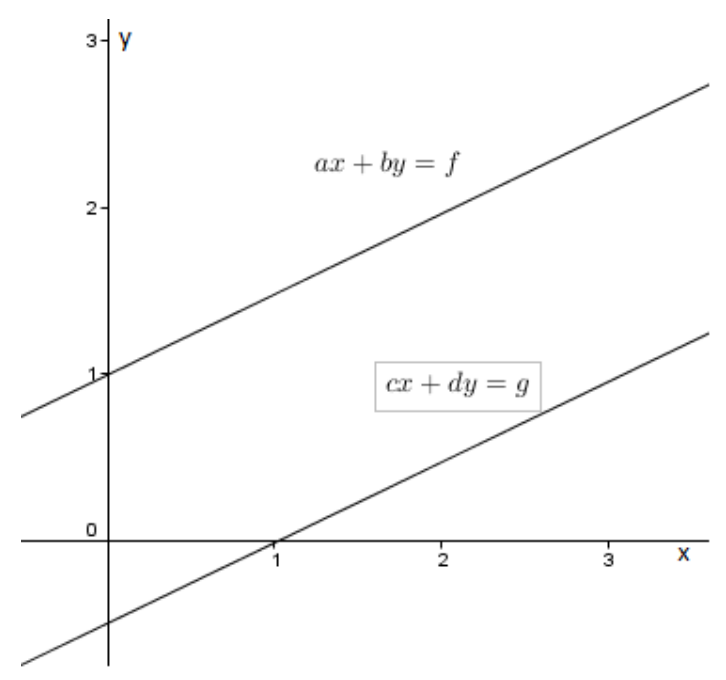

Figura 2.3: retas paralelas 


\subsection{Determinante de ordem 3}

Analogamente ao que fizemos no estudo do determinante de ordem 2, para reconstruirmos o determinante de ordem 3 utilizaremos um sistema linear de 3 equações e 3 incógnitas sobre $\mathbb{R}$, conjuntos dos números reais.

Vamos supor o seguinte sistema de equações:

$$
\left\{\begin{array}{c}
I: a x+b y+c z=j \\
I I: d x+e y+f z=k \\
I I I: g x+h y+i z=l
\end{array}\right.
$$

Considerando que $x, y$ e $z$ representam as incógnitas do sistema, que $a, d$ e $g$ representam os coeficientes de $x$, que $b, \mathrm{e}, h$ representam os coeficientes de $y$, que $c, f$ e $i$ representam os coeficientes de $z$ e que $j, k$ e $l$ representam os termos independentes do sistema, vamos algoritmizar a resolução do mesmo por meio do processo de eliminação de incógnitas.

Sem perda de generalidade, iremos eliminar sucessivamente as incógnitas $z$ e $y$ respectivamente e para isso multiplicaremos primeiro toda a segunda equação por $\frac{-c}{f}$ . De fato, para realizarmos essa operação devemos supor que $f \neq 0$, porém se $f=0$, faremos o mesmo processo só que tentando eliminar a incógnita $x$ ou $y$. Cabe salientar que $f, d$, e não podem ser ao mesmo tempo iguais a zero, pois se forem, teremos uma equação $0 x+0 y+0 z=k$ e esta terá solução única para $k=0$, porém isso restringiria o sistema a apenas duas equações e este caso não é o objeto atual do estudo proposto.

Supondo então $f \neq 0$, e multiplicando a equação II por $\frac{-c}{f}$, encontramos uma nova equação

$$
I I_{b}: \frac{-c d}{f} x-\frac{c e}{f} y-c z=\frac{-c k}{f} .
$$

Somando agora a equação $I$ com a equação $I I_{b}$, a incógnita $z$ é eliminada e a equação resultante pode ser desenvolvida da seguinte forma:

$$
x\left(a-\frac{c d}{f}\right)+y\left(b-\frac{c e}{f}\right)=j-\frac{c k}{f} \Rightarrow x\left(\frac{f a-c d}{f}\right)+y\left(\frac{f b-c e}{f}\right)=\frac{f j-c k}{f}
$$


Como no início supomos $f \neq 0$, podemos cancelar os denominadores, logo teremos uma nova expressão que chamaremos de equação IV nas incógnitas $x$ e $y$.

$$
I V: x(f a-c d)+y(f b-c e)=f j-c k
$$

Do mesmo modo que fizemos anteriormente, agora multiplicaremos a equação II por $\frac{-i}{f}$ a fim de somá-la a equação III e eliminar assim a incógnita $z$. De fato, como supomos $f \neq 0$, ao multiplicarmos a equação II por $\frac{-i}{f}$, encontraremos uma nova equação

$$
I I_{c}: \frac{-i d}{f} x-\frac{i e}{f} y-i z=\frac{-i k}{f} .
$$

Somando agora a equação $I I I$ com a equação $I I_{c}$, a incógnita $z$ é eliminada e a equação resultante pode ser desenvolvida da seguinte forma:

$$
x\left(g-\frac{i d}{f}\right)+y\left(h-\frac{i e}{f}\right)=l-\frac{i k}{f} \Rightarrow x\left(\frac{f g-i d}{f}\right)+y\left(\frac{f h-i e}{f}\right)=\frac{f l-i k}{f}
$$

Como no início supomos $f \neq 0$, podemos cancelar os denominadores, logo teremos uma nova expressão que chamaremos de equação $\mathrm{V}$ nas incógnitas $x$ e $y$

$$
V: x(f g-i d)+y(f h-i e)=f l-i k
$$

Neste momento do desenvolvimento nos deparamos com duas equações $I V$ e $V$, ambas nas incógnitas $x$ e $y$, ou seja, temos um sistema novo de duas equações e duas incógnitas, e como tal podemos resolvê-lo pela generalização de determinantes de ordem 2 obtida anteriormente.

$$
\left\{\begin{array}{c}
I V: x(f a-c d)+y(f b-c e)=f j-c k \\
V: x(f g-i d)+y(f h-i e)=f l-i k
\end{array}\right.
$$

Resolvendo o sistema pelo método dos determinantes de ordem 2, primeiramente devemos montar a matriz dos coeficientes de $x$ e $y$ a fim de calcular o Det, dessa forma temos 


$$
\text { Det }=\left|\begin{array}{cc}
(f a-c d) & (f b-c e) \\
(f g-i d) & (f h-i e)
\end{array}\right|=(f a-c d)(f h-i e)-(f b-c e)(f g-i d)
$$

Desenvolvendo a expressão temos

$$
\begin{gathered}
\text { Det }=\left(f^{2} a h-f a i e-f h c d+c d i e\right)-\left(f^{2} b g-f b i d-f g c e+c e i d\right) \\
\text { Det }=f^{2} a h-f a i e-f h c d+c d i e-f^{2} b g+f b i d+f g c e-c e i d \\
\text { Det }=f(f a h-a i e-h c d-f b g+b i d+g c e)
\end{gathered}
$$

Para calcular o determinante de $x$, devemos montar a matriz dos termos independentes e dos coeficientes de $y$, dessa forma temos

$$
\operatorname{Det}_{x}=\left|\begin{array}{cc}
(f j-c k) & (f b-c e) \\
(f l-i k) & (f h-i e)
\end{array}\right|=(f j-c k)(f h-i e)-(f b-c e)(f l-i k)
$$

Desenvolvendo a expressão temos

$$
\begin{gathered}
\text { Det }_{x}=\left(f^{2} j h-f j i e-f h c k+c k i e\right)-\left(f^{2} b l-f b i k-f l c e+c e i k\right) \\
\text { Det }_{x}=f^{2} j h-f j i e-f h c k+c k i e-f^{2} b l+f b i k+f l c e-c e i k \\
\text { Det }_{x}=f(f j h-j i e-h c k-f b l+b i k+l c e)
\end{gathered}
$$

Para calcular o determinante de $y$, devemos montar a matriz dos coeficientes de $x$ e dos termos independentes, dessa forma temos

$$
\operatorname{Det}_{y}=\left|\begin{array}{cc}
(f a-c d) & (f j-c k) \\
(f g-i d) & (f l-i k)
\end{array}\right|=(f a-c d)(f l-i k)-(f j-c k)(f g-i d)
$$

Desenvolvendo a expressão temos

$$
\text { Det }_{y}=\left(f^{2} a l-f a i k-f l c d+c d i k\right)-\left(f^{2} j g-f j i d-c k f g+c k i d\right)
$$




$$
\begin{array}{r}
\text { Det }_{y}=f^{2} a l-f a i k-f l c d+c d i k-f^{2} j g+f j i d+f g c k-c k i d \\
\text { Det }_{y}=f(f a l-a i k-l c d-f j g+j i d+g c k)
\end{array}
$$

Utilizando as relações $x($ Det $)=\operatorname{Det}_{x}$ e $y($ Det $)=$ Det $_{y}$, podemos calcular os valores de $x$ e $y$. Logo teremos

$$
x f(f a h-a i e-h c d-f b g+b i d+g c e)=f(f j h-j i e-h c k-f b l+b i k+l c e)
$$

Como no inicio supomos $f \neq 0$, podemos cancelar $f$ nos dois lados da igualdade, e multiplicando ambos termos por -1 chegamos a seguinte expressão

$$
x(a i e+h c d+f b g-b i d-g c e-f a h)=(j i e+h c k+f b l-b i k-l c e-f j h)
$$

Calculando agora o valor de $y$, temos

$$
y f(f a h-a i e-h c d-f b g+b i d+g c e)=f(f a l-a i k-l c d-f j g+j i d+g c k)
$$

Como no inicio supomos $f \neq 0$, podemos cancelar $f$ nos dois lados da igualdade, e multiplicando ambos termos por -1 chegamos a seguinte expressão

$$
y(a i e+h c d+f b g-b i d-g c e-f a h)=(a i k+l c d+f j g-j i d-g c k-f a l)
$$

Para calcularmos o valor de $z$, admitindo que o sistema seja possível e que existam $x$ e $y$ que os satisfaça de acordo com as expressões anteriormente vistas, vamos substituir os valores encontrados de $x$ e $y$ na equação II. Logo teremos 


$$
\begin{aligned}
& d\left(\frac{(j i e+h c k+f b l-b i k-l c e-f j h)}{(a i e+h c d+f b g-b i d-g c e-f a h)}\right)+e\left(\frac{(a i k+l c d+f j g-j i d-g c k-f a l)}{(a i e+h c d+f b g-b i d-g c e-f a h)}\right)+f z=k \\
& f z=k-d\left(\frac{(j i e+h c k+f b l-b i k-l c e-f j h)}{(a i e+h c d+f b g-b i d-g c e-f a h)}\right)-e\left(\frac{(a i k+l c d+f j g-j i d-g c k-f a l)}{(a i e+h c d+f b g-b i d-g c e-f a h)}\right) \\
& f z=\frac{(k a i e+k h c d+k f b g-k b i d-k g c e-k f a h-d j i e-d h c k-d f b l+d b i k+d l c e+d f j h-e a i k-e l c d-e f j g+e j i d+e g c k+e f a l)}{a i e+h c d+f b g-b i d-g c e-f a h}
\end{aligned}
$$

Cancelando os monômios semelhantes reduzimos a expressão para

$$
f z=\frac{(k f b g-k f a h-d f b l+d f j h-e f j g+e f a l)}{a i e+h c d+f b g-b i d-g c e-f a h} \Rightarrow f z=\frac{f(k b g-k a h-d b l+d j h-e j g+e a l)}{a i e+h c d+f b g-b i d-g c e-f a h}
$$

Finalmente, como supomos $f \neq 0$, podemos cancelar $f$ em ambos os membros e chegamos a expressão que soluciona a incógnita $z$ da seguinte forma

$$
z(a i e+h c d+f b g-b i d-g c e-f a h)=(k b g-k a h-d b l+d j h-e j g+e a l)
$$

Podemos observar que a expressão que funciona como coeficiente das três incógnitas do sistema é a mesma, e por esse motivo chamaremos esta expressão de determinante de ordem 3. Usaremos algumas vezes a expressão "Det" para nos referirmos ao valor determinante. Dessa forma temos

$$
\text { Det }=(a i e+h c d+f b g-b i d-g c e-f a h)
$$

Se agruparmos os coeficientes das incógnitas $x, y$ e $z$ respectivamente em uma matriz, observaremos que o Det é dado por uma relação entre os elementos que se alocam na diagonal principal e suas paralelas e uma relação entre os elementos que se alocam na diagonal secundária e em suas paralelas.

$$
\begin{gathered}
\text { Assim, na matriz }\left(\begin{array}{lll}
a & b & c \\
d & e & f \\
g & h & i
\end{array}\right) \text {, podemos afirmar que o determinante dessa matriz } \\
\text { é dado por } D e t=\left|\begin{array}{ccc}
a & b & c \\
d & e & f \\
g & h & i
\end{array}\right|=(\text { aie }+h c d+f b g-b i d-g c e-f a h) \text {. }
\end{gathered}
$$


Observamos ainda que para determinar o valor da incógnita $x$, não basta apenas o valor determinante $D e t$, precisaremos também da expressão $(j i e+h c k+f b l-b i k-$ $l c e-f j h)$. A esta expressão daremos o nome de determinante de $x$ e usaremos a notação

$$
\operatorname{Det}_{x}=(j i e+h c k+f b l-b i k-l c e-f j h) .
$$

De forma análoga, utilizaremos os coeficientes para formar uma matriz, porém trocaremos os coeficientes da incógnita $x$ pelos termos independentes do sistema, e assim podemos escrever que o determinante de $x$ é o Det da matriz obtida com os termos independentes, os coeficientes de $y$ e os coeficientes de $z$.

$$
\begin{aligned}
& \text { Assim, na matriz }\left(\begin{array}{ccc}
j & b & c \\
k & e & f \\
l & h & i
\end{array}\right) \text {, podemos afirmar que o determinante dessa matriz } \\
& \text { é dado por } \operatorname{Det}_{x}=\left|\begin{array}{ccc}
j & b & c \\
k & e & f \\
l & h & i
\end{array}\right|=(j i e+h c k+f b l-b i k-l c e-f j h) .
\end{aligned}
$$

Com o mesmo tipo de observação, podemos perceber que para determinar o valor da incógnita $y$, precisaremos da expressão $(a i k+l c d+f j g-j i d-g c k-f a l)$. A este valor daremos o nome de determinante de $y$ e usaremos a notação

$$
\operatorname{Det}_{y}=(a i k+l c d+f j g-j i d-g c k-f a l) .
$$

De forma análoga, utilizaremos os coeficientes para formar uma matriz, porém trocaremos os coeficientes da incógnita $y$ pelos termos independentes do sistema, e assim podemos escrever que o determinante de $y$ é o Det da matriz obtida com os coeficientes da incógnita $x$, os termos independentes e os coeficientes de $z$.

$$
\text { Assim, na matriz }\left(\begin{array}{ccc}
a & j & c \\
d & k & f \\
g & l & i
\end{array}\right) \text {, podemos afirmar que o determinante dessa matriz }
$$


é dado por Det $_{y}=\left|\begin{array}{lll}a & j & c \\ d & k & f \\ g & l & i\end{array}\right|=(a i k+l c d+f j g-j i d-g c k-f a l)$.

Da mesma maneira que fizemos para as incógnitas $x$ e $y$, podemos observar que para determinar o valor da incógnita $z$, precisaremos da expressão $(k b g-k a h-d b l+$ $d j h-e j g+e a l)$. A este valor daremos o nome de determinante de $z$ e usaremos a notação

$$
\operatorname{Det}_{z}=(k b g-k a h-d b l+d j h-e j g+e a l) .
$$

De forma análoga, utilizaremos os coeficientes para formar uma matriz, porém trocaremos os coeficientes da incógnita $z$ pelos termos independentes do sistema, e assim podemos escrever que o determinante de $z$ é o Det da matriz obtida com os coeficientes da incógnita $x$, os coeficientes da incógnita $y$ e os termos independentes.

Assim, na matriz $\left(\begin{array}{ccc}a & b & j \\ d & e & k \\ g & h & l\end{array}\right)$, podemos afirmar que o determinante dessa matriz é dado por $\operatorname{Det}_{z}=\left|\begin{array}{lll}a & b & j \\ d & e & k \\ g & h & l\end{array}\right|=(k b g-k a h-d b l+d j h-e j g+e a l)$.

Chegando a esse ponto do estudo podemos generalizar a solução do sistema de três equações e três incógnitas $x, y$ e $z$ da seguinte forma:

Dado o sistema linear de três equações e três incógnitas

$$
\left\{\begin{array}{l}
a x+b y+c z=j \\
d x+e y+f z=k \\
g x+h y+i z=l
\end{array}\right.
$$

as soluções do sistema podem ser genericamente expressas pelas relações

$$
x(\text { Det })=\operatorname{Det}_{x}, y(\operatorname{Det})=\operatorname{Det}_{y}, z(\operatorname{Det})=\operatorname{Det}_{z}
$$


onde,

Det é o determinante da matriz $\left(\begin{array}{ccc}a & b & c \\ d & e & f \\ g & h & i\end{array}\right)$ formada pelos coeficientes das incóg-

nitas $x, y$ e $z$ respectivamente e, Det $=\left|\begin{array}{lll}a & b & c \\ d & e & f \\ g & h & i\end{array}\right|=($ aie $+h c d+f b g-b i d-g c e-f a h)$;

Det $_{x}$ é o determinante da matriz $\left(\begin{array}{ccc}j & b & c \\ k & e & f \\ l & h & i\end{array}\right)$ formada pelos termos independen-

tes, pelos coeficientes da incógnita $y$ e pelos coeficientes da incógnita $z$ e, Det $t_{x}=$ $\left|\begin{array}{ccc}j & b & c \\ k & e & f \\ l & h & i\end{array}\right|=(j i e+h c k+f b l-b i k-l c e-f j h)$

Det $_{y}$ é o determinante da matriz $\left(\begin{array}{ccc}a & j & c \\ d & k & f \\ g & l & i\end{array}\right)$ formada pelos coeficientes da incógnita $x$, pelos termos independentes e pelos coeficientes da incógnita $z$ e, Det $_{y}=$ $\left|\begin{array}{lll}a & j & c \\ d & k & f \\ g & l & i\end{array}\right|=(a i k+l c d+f j g-j i d-g c k-f a l)$.

Det $_{z}$ é o determinante da matriz $\left(\begin{array}{ccc}a & b & j \\ d & e & k \\ g & h & l\end{array}\right)$ formada pelos coeficientes da incógnita $x$, pelos coeficientes da incógnita $y$ e pelos termos independentes e, 


$$
\operatorname{Det}_{z}=\left|\begin{array}{lll}
a & b & j \\
d & e & k \\
g & h & l
\end{array}\right|=(k b g-k a h-d b l+d j h-e j g+e a l) .
$$

Cabe salientar que como $x, y$ e $z$ são determinados por uma equação, esta pode assumir casos diferentes os quais abordaremos agora.

\subsubsection{Casos do Sistema}

$\mathbf{1}^{\circ} \mathrm{CASO}-($ Det $\neq 0)$

Sempre que o Det $\neq 0$, as expressões que solucionam $x, y$ e $z$ são possíveis e determinadas, logo o sistema terá solução possível e unicamente determinada pelo algorítimo de resolução anteriormente demonstrado.

$\mathbf{2}^{\mathbf{o}} \mathbf{C A S O}-\left(\operatorname{Det}=0, \operatorname{Det}_{x}=0, \operatorname{Det}_{y}=0, \operatorname{Det}_{z}=0\right)$

Sempre que todos os determinantes do sistema forem nulos, as expressões que determinam $x, y$ e $z$, a saber $0 x=0,0 y=0,0 z=0$ porerão ser solucionadas por diversos trios ordenados $(x, y, z)$, neste caso, o sistema será indeterminado e terá portanto infinitos trios de solução.

$3^{\mathbf{o}} \mathbf{C A S O}-\left(\operatorname{Det}=0, \operatorname{Det}_{x} \neq 0\right.$, ou $\operatorname{Det}_{y} \neq 0$, ou $\left.\operatorname{Det}_{z} \neq 0\right)$

Sempre que o determinante for nulo e que os determinantes de $x, y$ ou $z$ forem diferentes de zero, as expressões que determinam $x, y$ ou $z$ serão expressas por $0 x=$ $\operatorname{Det}_{x}, 0 y=\operatorname{Det}_{y}$ ou $0 z=$ Det $_{z}$ e nenhuma dessas equações possui solução possível e portanto o sistema será classificado como impossível.

\subsubsection{Indexando os Elementos da Matriz}

Assim como fizemos anteriormente no estudo dos determinantes de ordem 2 e utilizando as mesmas notações para matrizes e determinantes que utilizamos naquela seção, podemos também indexar os elementos da matriz de ordem 3 e com isso obtemos 


$$
\left(\begin{array}{lll}
a & b & c \\
d & e & f \\
g & h & i
\end{array}\right)=\left(\begin{array}{lll}
a_{11} & a_{12} & a_{13} \\
a_{21} & a_{22} & a_{23} \\
a_{31} & a_{32} & a_{33}
\end{array}\right)
$$

Podemos perceber que cada elemento $a_{i j}$ da matriz representa o elemento alocado na linha $i$ e na coluna $j$. Dessa forma pode-se estabelecer o determinante dessa matriz da seguinte forma.

$$
\begin{aligned}
& \text { Det }=\left|\begin{array}{ccc}
a_{11} & a_{12} & a_{13} \\
a_{21} & a_{22} & a_{23} \\
a_{31} & a_{32} & a_{33}
\end{array}\right|=\left(a_{11} a_{22} a_{33}\right)+\left(a_{12} a_{23} a_{31}\right)+\left(a_{13} a_{21} a_{32}\right)-\left(a_{31} a_{22} a_{13}\right)- \\
&\left(a_{32} a_{23} a_{11}\right)-\left(a_{33} a_{21} a_{12}\right)
\end{aligned}
$$

Uma maneira prática e fácil de usar e memorizar o determinante de matrizes de ordem três é conhecida como regra de Sarrus, em homenagem ao matemático francês Pierre Fréderic Sarrus.

\subsubsection{Regra de Sarrus}

A regra de Sarrus estabelece que para calcularmos o determinante de uma matriz de ordem três devemos seguir alguns passos.

$1^{\circ}$ passo: Montar a matriz $A$ repetindo a sua direita a primeira e segunda colunas como mostra o exemplo abaixo

$$
\left(\begin{array}{ccc|cc}
a_{11} & a_{12} & a_{13} & a_{11} & a_{12} \\
a_{21} & a_{22} & a_{23} & a_{21} & a_{22} \\
a_{31} & a_{32} & a_{33} & a_{31} & a_{32}
\end{array}\right)
$$

$2^{\circ}$ passo: Multiplicar os elementos da diagonal principal, depois multiplicar os elementos das diagonais paralelas a principal, e por ultimo somar os três resultados encontrados nas multiplicações das três diagonais. A esta relação daremos o nome de $\operatorname{Det} A_{p}$. 


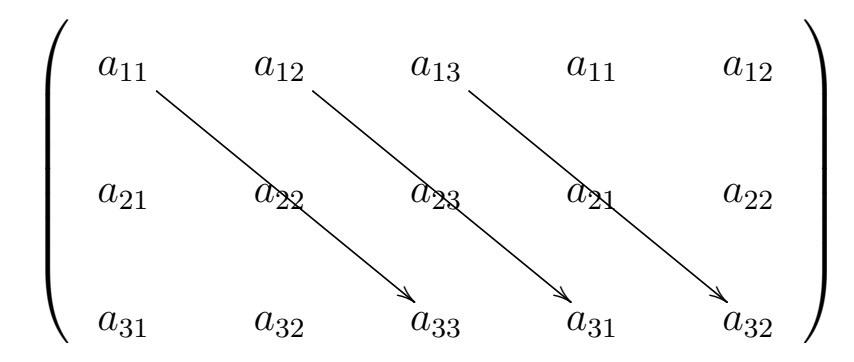

Det $A_{p}=\left(a_{11} a_{22} a_{33}\right)+\left(a_{12} a_{23} a_{31}\right)+\left(a_{13} a_{21} a_{32}\right)$

$3^{\circ}$ passo: Multiplicar os elementos da diagonal secundária, depois multiplicar os elementos das diagonais paralelas a secundária e por último somar os três resultados encontrados nas multiplicações das três diagonais. A esta relação daremos o nome de $\operatorname{Det} A_{s}$.

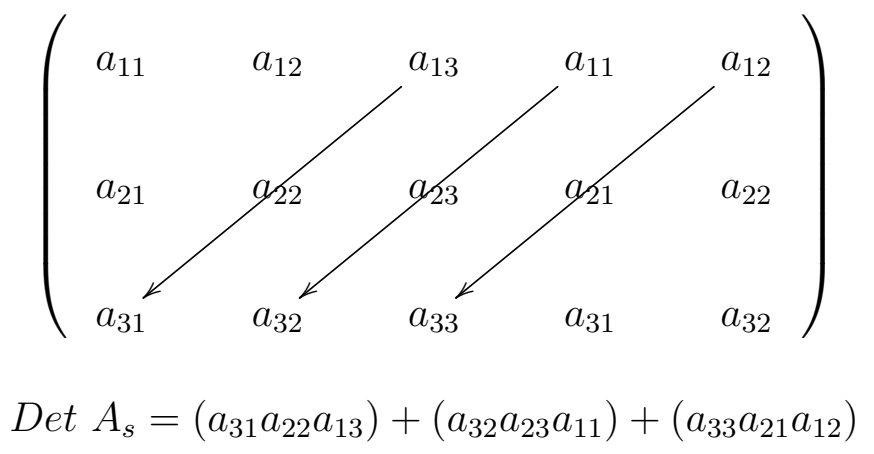

$4^{\circ}$ passo: $\mathrm{O}$ determinante de qualquer matriz de ordem 3 pode ser escrito como a diferença entre $\operatorname{Det} A_{p}$ e $\operatorname{Det} A_{s}$, ou seja,

$$
\begin{gathered}
\text { Det }=\operatorname{Det} A_{p}-\operatorname{Det} A_{s} \\
\text { Det }=\left[\left(a_{11} a_{22} a_{33}\right)+\left(a_{12} a_{23} a_{31}\right)+\left(a_{13} a_{21} a_{32}\right)\right]-\left[\left(a_{31} a_{22} a_{13}\right)+\left(a_{32} a_{23} a_{11}\right)+\left(a_{33} a_{21} a_{12}\right)\right]
\end{gathered}
$$




\subsubsection{Analogia Geométrica dos Casos do Sistema}

Cada um dos casos do sistema de três equações e três incógnitas que foram mostrados nesta subseção pode ser representado geometricamente por posições relativas entre três planos no espaço. Esta analogia atribui significado adicional ao entendimento dos casos do sistema e pode ter papel importante no trabalho com alunos do Ensino Médio caso sejam abordadas da forma e no momento corretos.

Como verificamos nas referências [4], [5], [6], [7] e [8], as escolas do país não trabalham o estudo das equações do plano no ensino médio, logo o professor deve tomar um cuidado adicional ao fazer essa analogia, pois seu aprofundamento não será possível nesta etapa de ensino. Acredito que o ideal seja de forma apenas ilustrativa e comparativa com os casos da reta anteriormente vistos, mencionar que cada uma das equações representam um plano no espaço e a seguir mostrar as figuras ilustrativas dos casos possíveis, salientando que um aprofundamento do tema será visto na universidade por aqueles que vierem a cursar algo relacionado as ciências exatas.

Nos parágrafos abaixo, abordamos de forma superficial uma analogia geométrica entre três planos no espaço a cada um dos casos que o sistema de três equações e três incógnitas pode assumir. Lembrando ainda que a solução de um sistema de três equações e três incógnitas é sempre a região do espaço que está contida na intersecção dos três planos, logo, seguindo esse raciocínio, só haverá solução para o sistema se houver uma intersecção comum entre os três planos.

\section{$\mathbf{1}^{\mathbf{O}} \mathrm{CASO}-($ Det $\neq 0)$}

Como ja vimos, neste caso o sistema possui solução unicamente determinada, ou seja, existe um único trio ordenado $(x, y, z)$ que soluciona o sistema. Essa solução única, neste caso específico, é representada por um ponto que esta contido na intersecção comum entre os três planos. Podemos observar de forma ilustrativa a representação geométrica deste caso na figura 2.4, para tal, observamos que só existe um ponto em todo o espaço que está contido ao mesmo tempo nos três planos do sistema 


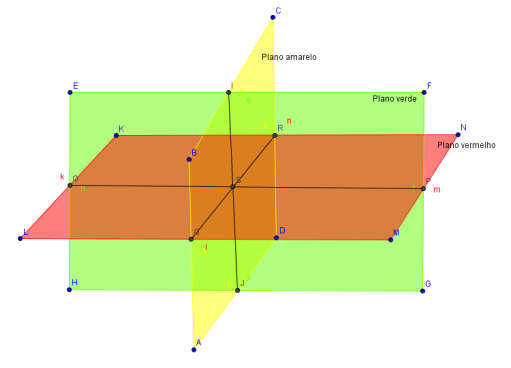

Figura 2.4: Planos cuja intersecção é um ponto

$\mathbf{2}^{\mathbf{o}} \mathbf{C A S O}-\left(\operatorname{Det}=0, \operatorname{Det}_{x}=0, \operatorname{Det}_{y}=0 \operatorname{Det}_{z}=0\right)$

Neste caso, o sistema possui infinitas soluções, ou seja, existem infinitos trios ordenados $(x, y, z)$ que solucionam este sistema. Todos estes trios ordenados, como são soluções do sistema, estão contidos na região do espaço que compreende a intersecção dos três planos.

Essa região, apesar de conter infinitos pontos, pode ter dois formatos diferentes, representados por uma reta ou por um plano.

A intersecção será uma reta quando os três planos forem concorrentes em uma única reta, e a intersecção será um plano quando as três equações representarem o mesmo plano no espaço, ou seja, os três planos são coincidentes.

No primeiro formato de intersecção, qualquer ponto $(x, y, z)$ contido na reta será solução do sistema, já no segundo formato de intersecção, qualquer ponto $(x, y, z)$ contido nos planos será solução do sistema. Neste segundo caso, para encontrar soluções para o sistema, basta escolher qualquer uma das equações e encontrar pontos que as satisfaçam pois estes pontos também serão soluções do sistema.

Podemos observar a representação geométrica deste caso em seus dois formatos nas figuras $2.5,2.6$ e 2.7 abaixo

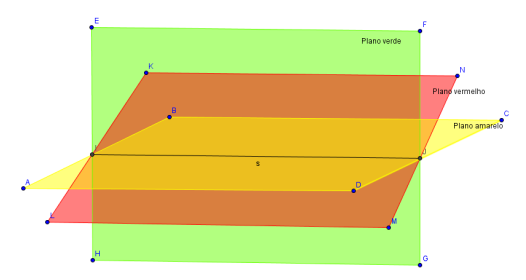

Figura 2.5: Planos cuja intersecção é uma reta 


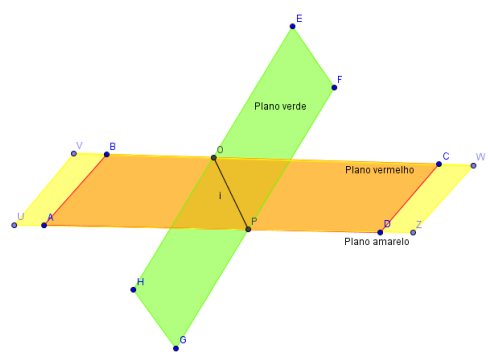

Figura 2.6: Planos cuja intersecção é uma reta 2

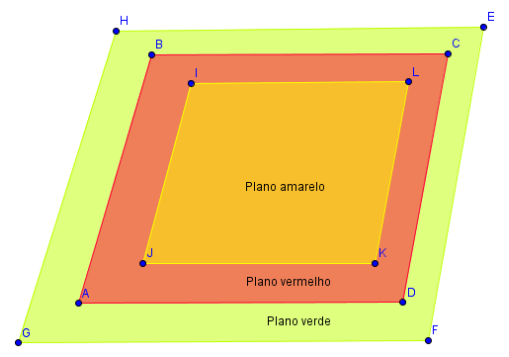

Figura 2.7: Planos cuja intersecção é um plano

$\mathbf{3}^{\mathbf{o}} \mathbf{C A S O}-\left(\operatorname{Det}=0, \operatorname{Det}_{x} \neq 0, \operatorname{Det}_{y} \neq 0 \operatorname{Det}_{z} \neq 0\right)$

Neste caso, não existe nenhuma solução para o sistema, ou seja, não existe nenhum trio ordenado $(x, y, z)$ que seja solução do sistema. Em outras palavras, não existe nenhuma região dos espaço que seja intersecção dos três planos representados pelas equações do sistema.

Existem várias combinações de posições relativas entre planos sem que haja uma intersecção entre os três planos. Nas figuras 2.8, 2.9 e 2.10 abaixo, podemos observar exemplos de posições entre os planos que podem representar este caso em questão.

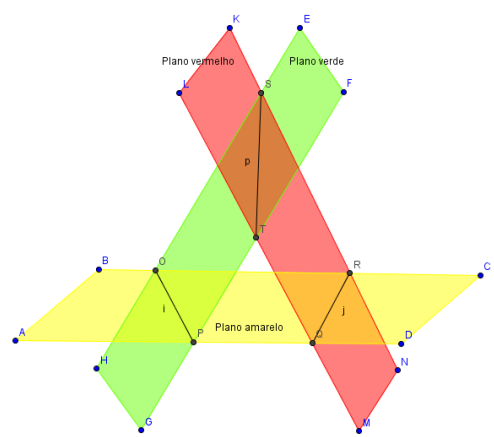

Figura 2.8: Planos sem intersecção tripla 


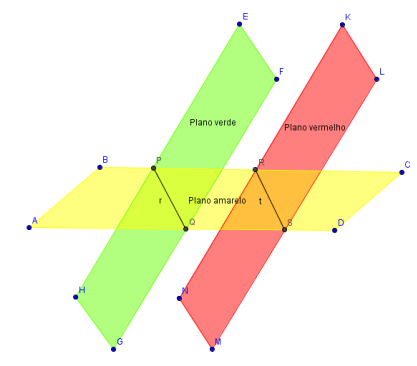

Figura 2.9: Planos sem intersecção tripla 2

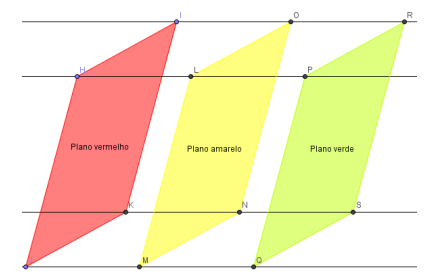

Figura 2.10: Planos sem intersecção tripla 3 


\section{Capítulo 3}

\section{Matrizes}

As definições e conceituações abordadas neste capítulo são fundamentadas nas referências de Hoffman e Kunze ([2], pp. 6-23) e sobre Iezzi e Hazzan ([1], pp. 44-72), sendo objetivo fundamental deste capítulo o embasamento de definições e conceituações que serão utilizadas a posterior, na fundamentação matemática principal deste trabalho.

Definição 3.1. Seja $i \in\{1,2, \cdots, m\}$ e $j \in\{1,2, \cdots, n\}$. Chama-se matriz de ordem $m$ por $n$ e representa-se sobre a notação $A=\left(a_{i j}\right)_{m \times n}$ a uma tabela organizada de elementos, neste trabalho restrito a números reais, dispostos em $m$ linhas e $n$ colunas, onde cada elemento $a_{i j}$ está alocado na intersecção da linha $i$ com a coluna $j$.

Por consequência, todas as matrizes abordadas ao longo deste trabalho são consideradas matrizes de elementos reais ou comumente denominadas matrizes sobre $\mathbb{R}$.

\subsection{Tipos de Matrizes}

A seguir veremos alguns tipos de matrizes, suas nomenclaturas e peculiaridades.

\section{Matriz Linha}

Toda matriz que possui uma única linha é chamada de matriz linha, ou seja, é toda a matriz de ordem $(1 \times n)$.

Exemplo 3.1. $A=\left(\begin{array}{lll}4 & 12 & 20\end{array}\right)$ 


\section{Matriz Coluna}

Toda a matriz que possui uma única coluna é chamada de matriz coluna, ou seja, é toda a matriz de ordem $(n \times 1)$

Exemplo 3.2. $A=\left(\begin{array}{c}3 \\ 7 \\ 21 \\ 5\end{array}\right)$

3. Matriz Quadrada

Toda a matriz que possui o mesmo número de linhas e de colunas é chamada de matriz quadrada, ou seja, é toda a matriz de ordem $(n \times n)$

Exemplo 3.3. $A=\left(\begin{array}{ll}3 & 17 \\ 4 & 12\end{array}\right)$

4. Matriz Nula

Uma matriz é considerada nula quando todos os seus elementos são iguais a zero, ou seja, a matriz $\left(a_{i j}\right)_{m \times n}$ onde $(1 \leq i \leq m)$ e $(1 \leq j \leq n)$ é nula se $a_{i j}=0$ para qualquer $i$ e $j$.

Exemplo 3.4. $A_{33}=\left(\begin{array}{ccc}0 & 0 & 0 \\ 0 & 0 & 0 \\ 0 & 0 & 0\end{array}\right)$

5. Matriz Triangular

Uma matriz quadrada é considerada triangular se todos os elementos acima ou abaixo da diagonal principal forem nulos. Podemos dizer que uma matriz $A=$ $\left(a_{i j}\right)_{n \times n}$ é triangular sempre que ocorrer uma das seguintes situações: Se $i<j$ então $a_{i j}=0$ ou se $i>j$ então $a_{i j}=0$.

Exemplo 3.5. $A_{33}=\left(\begin{array}{ccc}1 & 4 & 5 \\ 0 & 3 & 1 \\ 0 & 0 & 2\end{array}\right) \quad A_{44}=\left(\begin{array}{llll}1 & 0 & 0 & 0 \\ 4 & 3 & 0 & 0 \\ 7 & 8 & 2 & 0 \\ 3 & 1 & 4 & 9\end{array}\right)$ 


\section{Matriz Diagonal}

Uma matriz quadrada é chamada de diagonal sempre que todos os elementos não pertencentes a diagonal principal forem nulos. Podemos dizer que a matriz $A=\left(a_{i j}\right)_{n \times n}$ é diagonal sempre que ocorrer o seguinte: Se $i \neq j$ então $a_{i j}=0$.

Exemplo 3.6. $A_{33}=\left(\begin{array}{ccc}2 & 0 & 0 \\ 0 & 14 & 0 \\ 0 & 0 & 9\end{array}\right)$

\section{Matriz Identidade}

Uma matriz quadrada é chamada de identidade quando todos os elementos da diagonal principal forem iguais a 1 e todos os demais elementos forem nulos. Podemos dizer que a matriz $A=\left(a_{i j}\right)_{n \times n}$ é identidade sempre que obedecer simultaneamente a duas condições: 1) Se $i \neq j$ então $\left.a_{i j}=0 ; 2\right)$ Se $i=j$ então $a_{i j}=1$.

Exemplo 3.7. $I_{2}=\left(\begin{array}{ll}1 & 0 \\ 0 & 1\end{array}\right) \quad I_{3}=\left(\begin{array}{lll}1 & 0 & 0 \\ 0 & 1 & 0 \\ 0 & 0 & 1\end{array}\right) \quad I_{4}=\left(\begin{array}{llll}1 & 0 & 0 & 0 \\ 0 & 1 & 0 & 0 \\ 0 & 0 & 1 & 0 \\ 0 & 0 & 0 & 1\end{array}\right)$

\section{Matriz Simétrica}

Uma matriz quadrada $A=\left(a_{i j}\right)_{n \times n}$ é chamada de simétrica quando obedece a seguinte condição: $a_{i j}=a_{j i}$ para todo $i$ e todo $j$. Cabe salientar que após conhecermos a definição de matriz transposta que está apresentada no próximo item, decorrerá quase intuitivamente que se $A$ é uma matriz simétrica, então $A^{t}=A$.

Podemos observar no exemplo que em toda a matriz simétrica, os elementos simetricamente dispostos em relação a diagonal principal são iguais.

Exemplo 3.8. $A_{44}=\left(\begin{array}{cccc}2 & 5 & 6 & 8 \\ 5 & 0 & 1 & 7 \\ 6 & 1 & 3 & 4 \\ 8 & 7 & 4 & 9\end{array}\right)$ 


\section{Matriz Transposta}

Dada a matriz $A=\left(a_{i j}\right)_{m \times n}$, dizemos que a matriz $B=\left(b_{j i}\right)_{n \times m}$ é transposta de A se $a_{i j}=b_{j i}$ para todo $i$ e todo $j$ e denotamos por $A^{t}=B$ como a transposta da matriz $A$.

Exemplo 3.9. Dada $A=\left(\begin{array}{ccc}3 & -2 & 5 \\ 1 & 4 & 6\end{array}\right)$ temos $A^{t}=\left(\begin{array}{cc}3 & 1 \\ -2 & 4 \\ 5 & 6\end{array}\right)$

Podemos facilmente observar que se a matriz $A$ for simétrica então $A^{t}=A$.

Exemplo 3.10. Dada $A=\left(\begin{array}{ccc}4 & 2 & -1 \\ 2 & 5 & 9 \\ -1 & 9 & 3\end{array}\right)$ temos $A^{t}=\left(\begin{array}{ccc}4 & 2 & -1 \\ 2 & 5 & 9 \\ -1 & 9 & 3\end{array}\right)$

Associadas a ideia de matriz transposta estão algumas propriedades que apenas por fins didáticos e de organização, enunciaremos e demonstraremos a seguir quando falarmos de operações com matrizes.

Propriedade 3.1. $\left(A^{t}\right)^{t}=A$

A fim de demonstrar a propriedade, segue o seguinte. Seja $A=\left(a_{i j}\right)_{m \times n}$ uma matriz qualquer e seja $A^{t}=\left(b_{j i}\right)_{n \times m}$ tal que $b_{j i}=a_{i j}$ a sua matriz transposta. De fato, se $\left(A^{t}\right)^{t}=\left(c_{i j}\right)_{m \times n}$ tal que $c_{i j}=b_{j i}$ é a matriz trasposta de $A^{t}$ e observando que $c_{i j}=b_{j i}=a_{i j}$, então $\left(A^{t}\right)^{t}=A$.

\subsection{Operações com Matrizes}

\subsubsection{Adição de matrizes}

A adição de matrizes só pode ser efetuada quando as duas matrizes envolvidas na operação tiverem a mesma ordem. Caso essa condição seja atendida, basta adicionar os elementos correspondentes nas duas matrizes a fim de achar a matriz soma.

Seja $A=\left(a_{i j}\right)_{m \times n}$ e $B=\left(b_{i j}\right)_{m \times n}$ duas matrizes de mesma ordem. Podemos afirmar que a matriz soma de A com B é dada por $A+B=\left(a_{i j}+b_{i j}\right)_{m \times n}$. 
Exemplo 3.11. $\left(\begin{array}{cc}2 & 5 \\ -3 & 4 \\ 6 & 1\end{array}\right)+\left(\begin{array}{cc}3 & 2 \\ 7 & 1 \\ 2 & -4\end{array}\right)=\left(\begin{array}{cc}5 & 7 \\ 4 & 5 \\ 8 & -3\end{array}\right)$

Propriedade 3.2. Comutativa: $A+B=B+A$ para quaisquer matrizes $A$ e $B$ de ordem $m \times n$.

A fim de demonstrar a propriedade, segue o seguinte. Sendo $A=\left(a_{i j}\right)_{m \times n}$ e $B=$ $\left(b_{i j}\right)_{m \times n}$ duas matrizes de mesma ordem. Façamos $A+B=X$ e $B+A=Y$, sendo $x_{i j}=a_{i j}+b_{i j}$ e sendo $y_{i j}=b_{i j}+a_{i j}$, podemos afirmar que $x_{i j}=a_{i j}+b_{i j}=b_{i j}+a_{i j}=y_{i j}$ e com isso temos que $A+B=B+A$

Propriedade 3.3. Associativa: $(A+B)+C=A+(B+C)$ para quaisquer matrizes $A, B$ e $C$ de ordem $m \times n$.

A fim de demonstrar a propriedade, segue o seguinte. Sendo $A=\left(a_{i j}\right)_{m \times n}, B=$ $\left(b_{i j}\right)_{m \times n}$ e $C=\left(c_{i j}\right)_{m \times n}$ três matrizes de mesma ordem. Façamos $(A+B)+C=X$ e $A+(B+C)=Y$, sendo $x_{i j}=\left(a_{i j}+b_{i j}\right)+c_{i j}$ e sendo $y_{i j}=a_{i j}+\left(b_{i j}+c_{i j}\right)$, podemos afirmar que $x_{i j}=\left(a_{i j}+b_{i j}\right)+c_{i j}=a_{i j}+\left(b_{i j}+c_{i j}\right)=y_{i j}$ e com isso temos que $(A+B)+C=A+(B+C)$

Propriedade 3.4. Elemento Neutro: A matriz nula $0_{m \times n}$ satisfaz $A+0=A$, para toda matriz $A$ de ordem $m \times n$

A fim de demonstrar a propriedade, segue o seguinte. Sendo $A=\left(a_{i j}\right)_{m \times n}$ uma matriz qualquer, se existir um elemento neutro $E$ deve ser satisfeita a condição $A+E=$ $E+A=A$. Para que exista a adição $E$ deve ter a mesma ordem de $A$, logo podemos afirmar que $E=\left(e_{i j}\right)_{m \times n}$. Como $A+E=A$, temos que $a_{i j}+e_{i j}=a_{i j}, \operatorname{logo} e_{i j}=0$ , ou seja, cada elemento $e_{i j}$ da matriz $E$ é nulo e com isso podemos afirmar que o elemento neutro da adição é a matriz $E$ nula e de mesma ordem que a matriz $A$.

Propriedade 3.5. Elemento Simétrico: Qualquer que seja a matriz $A_{m \times n}$, existe uma matriz $-A_{m \times n}$ tal que $A+(-A)=0_{m \times n}$

A fim de demonstrar a propriedade, segue o seguinte. Sendo $A=\left(a_{i j}\right)_{m \times n}$ uma matriz qualquer, a propriedade do elemento simétrico diz que existe uma matriz $B$ tal que $A+B=0$. Para que exista a adição $B$ deve ter a mesma ordem de $A$, logo 
podemos afirmar que $B=\left(b_{i j}\right)_{m \times n}$. Como $A+B=0_{m \times n}$, temos que $a_{i j}+b_{i j}=0$, $\operatorname{logo} b_{i j}=-a_{i j}, \forall i$ e $\forall j$.

Dessa forma, podemos afirmar que a oposta da matriz $A$ em relação a adição é a matriz - $A$ da mesma ordem que $A$ e tal que cada elemento também é simétrico de seu correspondente em $A$.

A propriedade enunciada a seguir está intimamente ligada a ideia de matriz transposta.

Propriedade 3.6. $(A+B)^{t}=A^{t}+B^{t}$

A fim de demonstrar a propriedade, segue o seguinte. Seja $A=\left(a_{i j}\right)_{m \times n}$ e $B=$ $\left(b_{i j}\right)_{m \times n}$ duas matrizes quaisquer de mesma ordem. Sejam $A^{t}=\left(d_{j i}\right)_{n \times m}$ tal que $d_{j i}=a_{i j}$ e $B^{t}=\left(e_{j i}\right)_{n \times m}$ tal que $e_{j i}=b_{i j}$ respectivamente as matrizes transpostas de $A$ e $B$. Se $(A+B)^{t}=\left(c_{j i}\right)_{n \times m}$ tal que $c_{j i}=a_{i j}+b_{i j}$ é a matriz transposta de $A+B$, então podemos afirmar que $(A+B)^{t}=c_{j i}=a_{i j}+b_{i j}=d_{j i}+e_{j i}=A^{t}+B^{t}$.

\subsubsection{Multiplicação de um escalar por uma matriz}

A multiplicação de uma matriz por um escalar consiste no processo de multiplicar cada elemento dessa matriz por esse escalar.

Seja a matriz $A=\left(a_{i j}\right)_{m \times n}$ uma matriz qualquer e $k \in \mathbb{R}$ um escalar qualquer. Podemos afirmar que $k \cdot A_{m \times n}=\left(k \cdot a_{i j}\right)_{m \times n}$

\section{Exemplo 3.12.}

$$
3 \cdot\left(\begin{array}{ccc}
3 & 1 & 4 \\
-2 & 5 & 2
\end{array}\right)=\left(\begin{array}{ccc}
9 & 3 & 12 \\
-6 & 15 & 6
\end{array}\right)
$$

Dadas quaisquer matrizes de ordem $m \times n$ e números reais $p$ e $q$, podemos afirmar que valem as seguintes propriedades em relação a multiplicação por um escalar.

\section{Propriedade 3.7.}

i) $p(q A)=(p q) A$

ii) $(p+q) A=p A+q A$

iii) $p(A+B)=p A+p B$

iv) $1 \cdot A=A \cdot 1=A$ 
A propriedade enunciada a seguir está intimamente ligada a ideia de matriz transposta.

Propriedade 3.8. $(k \cdot A)^{t}=k \cdot A^{t}$

A fim de demonstrar a propriedade, segue o seguinte. Seja $A=\left(a_{i j}\right)_{m \times n}$ uma matriz qualquer e $A^{t}=\left(b_{j i}\right)_{n \times m}$ tal que $b_{j i}=a_{i j}$ a sua matriz transposta. Se $(k \cdot A)^{t}=\left(c_{j i}\right)_{n \cdot m}$ tal que $c_{j i}=k \cdot a_{i j}$ é a matriz trasnposta de $(k \cdot A)$ e observando que se $b_{j i}=a_{i j}$ então $k \cdot b_{j i}=k \cdot a_{i j}$, podemos afirmar que $(k \cdot A)^{t}=c_{j i}=k \cdot a_{i j}=k \cdot b_{j i}=k \cdot A^{t}$.

\subsubsection{Multiplicação de matrizes}

Para que duas matrizes possam ser multiplicadas, é condição necessária que o número de colunas da primeira matriz seja igual ao número de linhas da segunda, caso contrario, a multiplicação não poderá ser efetuada.

Uma vez atendida a condição necessária a multiplicação, a matriz resultante terá o mesmo número de linhas da primeira matriz e o mesmo número de colunas da segunda matriz.

Dessa forma, sendo $A=\left(a_{i j}\right)_{m \times n}$ e $B=\left(b_{j k}\right)_{n \times p}$ duas matrizes quaisquer, podemos definir a multiplicação $A_{m \times n} \cdot B_{n \times p}$ como sendo a matriz $C=\left(c_{i k}\right)_{m \times p}$ tal que:

$$
c_{i k}=\sum_{j=1}^{n} a_{i j} \cdot b_{j k}, \text { onde } 1 \leq i \leq m \text { e } 1 \leq k \leq p
$$

Exemplo 3.13. Dadas as matrizes $A_{2 \times 3}=\left(\begin{array}{ccc}2 & 3 & 5 \\ 3 & 4 & 2\end{array}\right)$ e $B_{3 \times 1}=\left(\begin{array}{l}2 \\ 6 \\ 3\end{array}\right)$ podemos afirmar que o produto $A_{2 \times 3} \cdot B_{3 \times 1}=C_{2 \times 1}=c_{i k}$, onde:

$$
\begin{aligned}
& c_{11}=a_{11} \cdot b_{11}+a_{12} \cdot b_{21}+a_{13} \cdot b_{31} \\
& c_{11}=2 \cdot 2+3 \cdot 6+5 \cdot 3=37 \\
& c_{21}=a_{21} \cdot b_{11}+a_{22} \cdot b_{21}+a_{23} \cdot b_{31} \\
& c_{21}=3 \cdot 2+4 \cdot 6+2 \cdot 3=36 \\
& \text { portanto a matriz } C_{2 \times 1}=\left(\begin{array}{l}
37 \\
36
\end{array}\right)
\end{aligned}
$$


Teorema 3.1. Sendo $A=\left(a_{i j}\right)_{m \times n}$ uma matriz qualquer, temos que $A I_{n}=A$ e que $I_{m} A=A$

Demonstração. Sendo $I_{n}=\left(d_{j k}\right)_{n \times n}$ e $B=A I_{n}=\left(b_{i k}\right)_{m \times n}$, temos que

$$
\begin{aligned}
b_{i k} & =a_{i 1} d_{1 k}+a_{i 2} d_{2 k}+a_{i 3} d_{3 k}+\cdots+a_{i k} d_{k k}+\cdots+a_{i n} d_{n k} \\
b_{i k} & =a_{i 1} 0+a_{i 2} 0+a_{i 3} 0+\cdots+a_{i k} 1+\cdots+a_{i n} 0=a_{i k} \text { para todo } i \text { e } k \text {, então } \\
A I_{n}= & A
\end{aligned}
$$

A demonstração de $I_{m} A=A$ se dá de forma análoga a esta, portanto deixaremos a mesma subentendida.

Propriedade 3.9. Associativa: Dadas as matrizes $A=\left(a_{i j}\right)_{m \times n}, B=\left(b_{i j}\right)_{n \times p}$ e $C=\left(c_{i j}\right)_{p \times q}$ podemos afirmar que $\left(A_{m \times n} \cdot B_{n \times p}\right) \cdot C_{p \times q}=A_{m \times n} \cdot\left(B_{n \times p} \cdot C_{p \times q}\right)$

A fim de demonstrar a propriedade, segue o seguinte. Seja $D=A B=\left(d_{i k}\right)_{m \times p}$, $E=(A B) C=\left(e_{i l}\right)_{m \times r}$, e $F=B C=\left(f_{j l}\right)_{n \times r}$, então:

$$
\begin{gathered}
e_{i l}=\sum_{k=1}^{p} d_{i k} \cdot c_{k l}=\sum_{k=1}^{p}\left(\sum_{j=1}^{n} a_{i j} \cdot b_{j k}\right) \cdot c_{k l} \\
e_{i l}=\sum_{k=1}^{p}\left(\sum_{j=1}^{n} a_{i j} \cdot b_{j k} \cdot c_{k l}\right)=\sum_{j=1}^{n} a_{i j} \cdot\left(\sum_{k=1}^{p} b_{j k} \cdot c_{k l}\right) \\
e_{i l}=\sum_{j=1}^{n} a_{i j} \cdot f_{j l}
\end{gathered}
$$

$\operatorname{logo},(A B) C=A(B C)$

Propriedade 3.10. Distributiva a Direita: Dadas as matrizes $A=\left(a_{i j}\right)_{n \times p}, B=$ $\left(b_{i j}\right)_{m \times n}$ e $C=\left(c_{i j}\right)_{m \times n}$ podemos afirmar que $(B+C) \cdot A=B \cdot A+C \cdot A$

A fim de demonstrar a propriedade, segue o seguinte. Seja $D=(A+B) C=$ $\left(d_{i k}\right)_{m \times p}$, então

$$
\begin{gathered}
d_{i k}=\sum_{j=1}^{n}\left(a_{i j}+b_{i j}\right) \cdot c_{j k}=\sum_{j=1}^{n}\left(a_{i j} \cdot c_{j k}+b_{i j} \cdot c_{j k}\right) \\
d_{i k}=\sum_{j=1}^{n} a_{i j} \cdot c_{j k}+\sum_{j=1}^{n} b_{i j} \cdot c_{j k}
\end{gathered}
$$

$\operatorname{logo}(A+B) C=A C+B C$. 
Propriedade 3.11. Distributiva a esquerda: Dadas as matrizes $A=\left(a_{i j}\right)_{p \times m}, B=$ $\left(b_{i j}\right)_{m \times n}$ e $C=\left(c_{i j}\right)_{m \times n}$ podemos afirmar que $A \cdot(B+C)=A \cdot B+A \cdot C$

A demonstração dessa propriedade é feita analogamente a propriedade anterior.

Propriedade 3.12. Dadas as matrizes $A=\left(a_{i j}\right)_{m \times n}$ e $B=\left(b_{j k}\right)_{n \times p}$ e sendo $q \in \mathbb{R}$ um escalar qualquer, podemos afirmar que $(q A) B=A(q B)=q(A B)$

A fim de demonstrar a propriedade, segue o seguinte. Seja $C=k A=\left(c_{i j}\right)_{m \times n}$, $D=k B=\left(d_{j k}\right)_{n \times p}$ e $E=A B=\left(e_{i k}\right)_{m \times p}$, então

$$
\begin{aligned}
& \sum_{j=1}^{n} c_{i j} \cdot b_{j k}=\sum_{j=1}^{n}\left(k \cdot a_{i j}\right) \cdot b_{j k}=k \cdot \sum_{j=1}^{n} a_{i j} \cdot b_{j k} \\
& \sum_{j=1}^{n} a_{i j} \cdot d_{j k}=\sum_{j=1}^{n} a_{i j} \cdot\left(k \cdot b_{j k}\right)=k \cdot \sum_{j=1}^{n} a_{i j} \cdot b_{j k}
\end{aligned}
$$

$\operatorname{logo},(k A) B=A(k B)=k(A B)$

A propriedade enunciada a seguir está intimamente ligada a ideia de matriz transposta.

Propriedade 3.13. Se $A=\left(a_{i j}\right)_{m \times n}$ e $B=\left(b_{i j}\right)_{n \times p}$, então $(A B)^{t}=B^{t} A^{t}$

A fim de demonstrar a propriedade, segue o seguinte. Seja $A B=C=\left(c_{i k}\right)_{m \times p} \mathrm{e}$ $(A B)^{t}=C^{t}=\left(d_{k i}\right)_{p \times m}, \log \mathrm{O}$

$$
c_{k i}^{\prime}=c_{i k}=\sum_{j=1}^{n} a_{i j} \cdot b_{j k}=\sum_{j=1}^{n} b_{j k} \cdot a_{i j}=\sum_{j=1}^{n} b_{k j}^{\prime} \cdot a_{j i}^{\prime}
$$




\section{Capítulo 4}

\section{Fundamentação Teórica do}

\section{Determinante}

Neste capítulo, trazemos uma abordagem mais conceitual e técnica ao estudo dos determinantes. Definiremos uma função que relaciona uma matriz real quadrada de ordem $n$ a um valor real que sempre pode ser obtido por uma combinação dos elementos dessa matriz. A essa função que definiremos, daremos o nome de função determinante.

Provaremos que sendo $n$ um inteiro positivo, sempre existe uma função determinante associada as matrizes de ordem $n$.

Por último, mostraremos que sendo $n$ um inteiro positivo, a função determinante associada as matrizes de ordem $n$ é única, ou seja, só existe uma função determinante associada as matrizes de ordem $n$.

Como o objetivo deste trabalho é o estudo dos determinantes e suas aplicações no Ensino Médio, tratamos apenas das matrizes formadas por elemento reais. Sendo assim, todas as matrizes consideradas são matrizes com entradas em $\mathbb{R}$.

As definições e conceituações abordadas neste capítulo, bem como a maioria das demonstrações são fundamentadas nas referências de Hoffman e Kunze ([2], pp. 138-163) e sobre Iezzi e Hazzan ([1], pp. 78-115) e seu objetivo fundamental é a demonstração da existência e da unicidade da função determinante para melhoria do entendimento deste assunto pelos professores que atuam no Ensino Médio e por conseguinte a melhoria no processo ensino e aprendizagem, não sendo portanto objetivo deste o estudo das diversas aplicações que o determinante pode nos levar, apesar de reconhecida a 
importância e abrangência que essas aplicações ensejam.

\subsection{Funções Determinantes}

Definição 4.1. Seja $n$ um inteiro positivo, $A=\left(a_{i j}\right)_{n \times n}$ uma matriz quadrada de ordem $n$, e seja $D$ uma função que associa a cada matriz $A$ um número real $D(A)$. Dizemos que a função $D$ é $n$-linear se para cada $i \in\{1,2, \cdots, n\}, D$ é uma função linear da $i$-ésima linha quando as outras $(n-1)$ linhas são mantidas fixas.

Digamos que $D$ seja uma função das matrizes $A=\left(a_{i j}\right)_{n \times n}$ e que $\alpha_{1}, \alpha_{2}, \cdots, \alpha_{n}$ sejam as linhas da matriz $A$. Podemos dizer então que $D$ é uma função das linhas de $A$ e representá-la por

$$
D(A)=D\left(\alpha_{1}, \alpha_{2}, \cdots, \alpha_{n}\right)
$$

Como definimos que $D$ é $n$-linear em relação as linhas de $A$, então temos

$$
D\left(\alpha_{1}, \cdots, c \alpha_{i}+\alpha_{i}, \cdots, \alpha_{n}\right)=c D\left(\alpha_{1}, \cdots, c \alpha_{i}, \cdots, \alpha_{n}\right)+D\left(\alpha_{1}, \cdots, \alpha_{i}^{\prime}, \cdots, \alpha_{n}\right)
$$

Como a função $D$ é $n$-linear, podemos dizer que $D$ é uma função da $i$-ésima linha enquanto as outras são fixas e com isso abreviar a função para $D(A)=D\left(\alpha_{i}\right)$. Dessa forma temos que

$$
D\left(c \alpha_{i}+\alpha_{i}^{\prime}\right)=c D\left(\alpha_{i}\right)+D\left(\alpha_{i}^{\prime}\right)
$$

Vamos a partir de agora mostrar alguns exemplos de funções $n$-lineares, que também serão úteis ainda nesta seção para algumas demonstrações.

Exemplo 4.1. Sejam $k_{1}, k_{2}, \cdots, k_{n}$ inteiros positivos tais que $k_{i} \in\{1,2, \cdots, n\}$ e seja $q$ um valor real. Para cada matriz $A=\left(a_{i j}\right)_{n \times n}$ definamos a função $D$ onde:

$$
D(A)=q \cdot a_{1 k_{1}} \cdot a_{2 k_{2}} \cdots a_{n k_{n}}
$$


Note que $D$ é obtida pelo produto de um elemento de cada linha distinta de $A$ e uma constante $q$. Queremos agora mostrar que essa função $D$ é $n$-linear.

Para isso, considere $D$ como uma função da $i$-ésima linha de A, mantidas as outras fixas. Então, como os $a_{r k_{r}}$ estão fixados para $r \neq i$, podemos escrever $D\left(\alpha_{i}\right)=b \cdot a_{\left(i k_{i}\right)}$ onde $b$ é o produto dos elementos $q$ e $a_{r k_{r}}$ tais que $r \neq i$.

Seja $\alpha_{i}^{\prime}=\left(a_{i 1}^{\prime}, a_{i 2}^{\prime}, \cdots, a_{i n}^{\prime}\right)$. Então

$$
D\left(c \alpha_{i}+\alpha_{i}^{\prime}\right)=\left(c \cdot a_{i k_{i}}+a_{i k_{i}}^{\prime}\right) \cdot b=c \cdot b \cdot a_{i k_{i}}+b \cdot a_{i k_{i}}^{\prime}=c \cdot D\left(\alpha_{i}\right)+D\left(\alpha_{i}^{\prime}\right)
$$

Dessa forma, concluímos que a função $D$ é uma função $n$-linear. Um caso especial de funções $D$ desse tipo é a função produto dos elementos da diagonal principal da matriz.

$$
D(A)=a_{11} \cdot a_{22} \cdot a_{33} \cdots a_{n n}
$$

Exemplo 4.2. Neste exemplo, vamos caracterizar as funções bilineares sobre as matrizes $A=\left(a_{i j}\right)_{2 \times 2}$. Digamos que a função $D$ seja uma dessas funções bilineares e seja $\epsilon_{1}$ e $\epsilon_{2}$ as linhas da matriz identidade $I_{2}=\left(\begin{array}{ll}1 & 0 \\ 0 & 1\end{array}\right)$.

Dessa forma, podemos escrever a matriz $A$ e a função $D$ como:

$$
\begin{gathered}
D(A)=D\left(a_{11} \epsilon_{1}+a_{12} \epsilon_{2}, a_{21} \epsilon_{1}+a_{22} \epsilon_{2}\right) \\
D(A)=D\left(a_{11} \epsilon_{1}, a_{21} \epsilon_{1}+a_{22} \epsilon_{2}\right)+D\left(a_{12} \epsilon_{2}, a_{21} \epsilon_{1}+a_{22} \epsilon_{2}\right) \\
D(A)=D\left(a_{11} \epsilon_{1}, a_{21} \epsilon_{1}\right)+D\left(a_{11} \epsilon_{1}, a_{22} \epsilon_{2}\right)+D\left(a_{12} \epsilon_{2}, a_{21} \epsilon_{1}\right)+D\left(a_{12} \epsilon_{2}, a_{22} \epsilon_{2}\right) \\
D(A)=a_{11} a_{21} D\left(\epsilon_{1}, \epsilon_{1}\right)+a_{11} a_{22} D\left(\epsilon_{1}, \epsilon_{2}\right)+a_{12} a_{21} D\left(\epsilon_{2}, \epsilon_{1}\right)+a_{12} a_{22} D\left(\epsilon_{2}, \epsilon_{2}\right)
\end{gathered}
$$

Desse modo, podemos verificar que a função bilinear $D$ é totalmente determinada por quatro escalares $D\left(\epsilon_{1}, \epsilon_{1}\right), D\left(\epsilon_{1}, \epsilon_{2}\right), D\left(\epsilon_{2}, \epsilon_{1}\right)$ e $D\left(\epsilon_{2}, \epsilon_{2}\right)$.

Lema 4.1. Uma combinação linear de funções $n$-lineares é $n$-linear.

Demonstração. Sejam $D$ e $E$ duas funções $n$-lineares sobre as matrizes $A=\left(a_{i j}\right)_{n \times n}$. Podemos escrever uma combinação linear de $D$ e $E$ como $p D+q E$, com $p$ e $q$ números reais. Dessa forma temos $(p D+q E)(A)=p D(A)+q E(A)$. Se fixarmos todas as linhas de $A$ exceto a linha $i$, teremos

$$
(p D+q E)\left(c \alpha_{i}+\alpha_{i}^{\prime}\right)=p D\left(c \alpha_{i}+\alpha_{i}^{\prime}\right)+q E\left(c \alpha_{i}+\alpha_{i}^{\prime}\right)
$$




$$
\begin{gathered}
(p D+q E)\left(c \alpha_{i}+\alpha_{i}^{\prime}\right)=p c D\left(\alpha_{i}\right)+p D\left(\alpha_{i}^{\prime}\right)+q c E\left(\alpha_{i}\right)+q E\left(\alpha_{i}^{\prime}\right) \\
(p D+q E)\left(c \alpha_{i}+\alpha_{i}^{\prime}\right)=c(p D+q E)\left(\alpha_{i}\right)+(p D+q E)\left(\alpha_{i}^{\prime}\right)
\end{gathered}
$$

Exemplo 4.3. Seja a função $D$ sobre a matriz $A=\left(a_{i j}\right)_{2 \times 2}$ tal que

$$
D(A)=a_{11} a_{22}-a_{12} a_{21}
$$

Se observarmos essa função com atenção, podemos perceber que ela é uma combinação linear de duas funções bilineares como no Exemplo 4.1.

Tomando $D_{1}=a_{11} a_{22}$ e $D_{2}=-a_{12} a_{21}$, a função $D=D_{1}+D_{2}$ é exatamente a função $D(A)=a_{11} a_{22}-a_{12} a_{21}$.

Como vimos no lema acima, uma combinação linear de funções $n$-lineares é $n$-linear, logo a função $D$ é bilinear.

Essa função $D$ vista no Exemplo 4.3 possui três propriedades muito interessantes que iremos citar e demonstrar a seguir:

1. Se $I_{2}$ é a matriz identidade de segunda ordem, então $D\left(I_{2}\right)=1$;

2. Se as duas linhas da matriz $A$ são iguais, então $D(A)=0$;

3. Se $A^{\prime}$ é a matriz obtida permutando as duas linhas de $A$, então $D\left(A^{\prime}\right)=-D(A)$

Para demonstrar 1 , se $I_{2}=\left(\begin{array}{cc}1 & 0 \\ 0 & 1\end{array}\right)$, temos que $D\left(I_{2}\right)=1 \cdot 1-0 \cdot 0=1$;

Agora, para demonstrar 2, se a matriz $A$ tem duas linhas iguais, então $a_{11}=a_{21} \mathrm{e}$ $a_{12}=a_{22}$. Com isso podemos escrever a função $D$ como $D(A)=a_{11} a_{12}-a_{11} a_{12}=0$;

Por fim, para demonstrar 3, se $A^{\prime}$ é a matriz obtida permutando as duas linhas da matriz $A$, então $A^{\prime}=\left(\begin{array}{cc}a_{21} & a_{22} \\ a_{11} & a_{12}\end{array}\right)$. Com isso, podemos escrever a função $D$ como $D\left(A^{\prime}\right)=a_{12} a_{21}-a_{11} a_{22}=-D(A)$.

Essas três propriedades serão utilizadas na definição da função determinante que abordaremos em breve ainda nesta seção. 
Definição 4.2. Seja $D$ uma função n-linear, dizemos que a função $D$ é alternada se duas condições são satisfeitas.

1. $D(A)=0$, sempre que $A$ tiver duas linhas iguais;

2. Se $A^{\prime}$ é uma matriz obtida permutando duas linhas quaisquer de $A$, então $D\left(A^{\prime}\right)=$ $-D(A)$.

Mais a frente, demonstraremos que qualquer função $n$-linear que satisfaça a condição 1, também satisfará a condição 2.

Definição 4.3. Seja $n$ um inteiro positivo, $I_{n}$ a matriz identidade de ordem $n$ e seja $A=\left(a_{i j}\right)_{n \times n}$ uma matriz quadrada de ordem $n$. Dizemos que uma função $D$ aplicada a matriz $A$ é uma função determinante, quando as seguintes condições forem satisfeitas.

1. $D$ é uma função $n$-linear;

2. $D$ é uma função alternada;

3. $D\left(I_{n}\right)=1$

Observe que a função $D$ descrita no Exemplo 4.3 atende às três condições, logo podemos dizer que a função do exemplo é uma função determinante.

Mostraremos a seguir que a função $D$ descrita no Exemplo 4.3 é a única função determinante bilinear que existe.

Lema 4.2. Se a função $D$ definida sobre as matrizes $A=\left(a_{i j}\right)_{2 \times 2}$ é uma função determinante, então a função $D$ é única e pode ser expressa como $D(A)=a_{11} a_{22}-$ $a_{12} a_{21}$.

Demonstração. Como vimos no Exemplo 4.2, se uma função $D$ é bilinear, então

$$
D(A)=a_{11} a_{21} D\left(\epsilon_{1}, \epsilon_{1}\right)+a_{11} a_{22} D\left(\epsilon_{1}, \epsilon_{2}\right)+a_{12} a_{21} D\left(\epsilon_{2}, \epsilon_{1}\right)+a_{12} a_{22} D\left(\epsilon_{2}, \epsilon_{2}\right)
$$

Digamos que uma função $D$ bilinear é também uma função determinante, então $D$ é alternada o que implica que $D\left(\epsilon_{1}, \epsilon_{1}\right)=0$ e $D\left(\epsilon_{2}, \epsilon_{2}\right)=0$. Com isso podemos reescrever $D$ como $D(A)=a_{11} a_{22} D\left(\epsilon_{1}, \epsilon_{2}\right)+a_{12} a_{21} D\left(\epsilon_{2}, \epsilon_{1}\right)$.

Como $D$ é uma função determinante, $D\left(I_{2}\right)=1$, logo $D\left(\epsilon_{1}, \epsilon_{2}\right)=1$, por outro lado, $D$ tambem é alternada, $\log o D\left(\epsilon_{2}, \epsilon_{1}\right)=-1$ o que nos permite reescrever a função $D$ como

$$
D(A)=a_{11} a_{22}-a_{12} a_{21}
$$


Dessa forma chegamos a conclusão que a função $D$ é a única função determinante de segunda ordem que existe.

Sempre que for necessário, podemos escrever uma matriz $A$ qualquer de ordem $n$ como o produto de elementos da matriz $A$ por linhas da matriz identidade de ordem $n$. Esse processo pode ser conveniente a fim de realizarmos algumas observações sobre funções aplicadas a matriz $A$ como veremos a seguir.

Exemplo 4.4. Seja $A=\left(a_{i j}\right)_{3 \times 3}$ uma matriz qualquer, onde $A=\left(\begin{array}{ccc}a_{11} & a_{12} & a_{13} \\ a_{21} & a_{22} & a_{23} \\ a_{31} & a_{32} & a_{33}\end{array}\right)$. Seja ainda $\epsilon_{1}, \epsilon_{2}, \epsilon_{3}$ as três linhas da matriz identidade $I_{3}$. Podemos escrever a matriz $A$ da seguinte forma

$$
A=\left(a_{11} \epsilon_{1}+a_{12} \epsilon_{2}+a_{13} \epsilon_{3}, a_{21} \epsilon_{1}+a_{22} \epsilon_{2}+a_{23} \epsilon_{3}, a_{31} \epsilon_{1}+a_{32} \epsilon_{2}+a_{33} \epsilon_{3}\right) .
$$

Lema 4.3. Seja $D$ uma função bilinear sobre as matrizes $A=\left(a_{i j}\right)_{2 \times 2}$ tal que $D(A)=0$ sempre que $A$ tem duas linhas iguais, então a função $D$ é alternada.

Demonstração. Para provar que $D$ é alternada, basta provar que dada uma matriz $A^{\prime}$ obtida pela permutação das linhas de $A$, temos $D\left(A^{\prime}\right)=-D(A)$.

Seja $\alpha, \beta$ duas linhas de uma matriz arbitraria $2 \times 2$. Como $D$ é bilinear, temos $D(\alpha+\beta, \alpha+\beta)=D(\alpha, \alpha)+D(\alpha, \beta)+D(\beta, \alpha)+D(\beta, \beta)$. Como por hipótese, $D(A)=0$ sempre que $A$ tem duas linhas iguais, temos então que $D(\alpha+\beta, \alpha+\beta)=D(\alpha, \alpha)=$ $D(\beta, \beta)=0$.

Com isso, reduzimos a equação para $D(\alpha, \beta)+D(\beta, \alpha)=0$, logo concluímos que $D(\alpha, \beta)=-D(\beta, \alpha)$, ou seja $D\left(A^{\prime}\right)=-D(A)$ sempre que $A^{\prime}$ é obtida permutando as linhas de $A$.

Lema 4.4. Seja $D$ uma função $n$-linear sobre as uma matrizes $A=\left(a_{i j}\right)_{n \times n}$. Se $D(A)=0$ sempre que $A$ tem duas linhas consecutivas iguais, então $D$ é uma função alternada.

Demonstração. Para provar que a função $D$ é alternada, precisamos mostrar que:

i) $D\left(A^{\prime}\right)=-D(A)$ sempre que $A^{\prime}$ é obtida permutando duas linhas quaisquer de $A$;

ii) $D(A)=0$ sempre que $A$ tenha duas linhas quaisquer iguais. 
i) Primeiro suponha que $A^{\prime}$ seja obtida pela permutação de duas linhas consecutivas de $A$. Seguindo o mesmo procedimento descrito no lema anterior, como $D$ é $n$-linear, podemos escrever

$$
\begin{aligned}
& D\left(\alpha_{1}, \cdots, \alpha_{i}+\beta_{i}, \alpha_{i}+\beta_{i}, \cdots, \alpha_{n}\right)=D\left(\alpha_{1}, \cdots, \alpha_{i}, \alpha_{i}, \cdots, \alpha_{n}\right)+D\left(\alpha_{1}, \cdots, \alpha_{i}, \beta_{i}, \cdots, \alpha_{n}\right) \\
& +D\left(\alpha_{1}, \cdots, \beta_{i}, \alpha_{i}, \cdots, \alpha_{n}\right)+D\left(\alpha_{1}, \cdots, \beta_{i}, \beta_{i}, \cdots, \alpha_{n}\right) .
\end{aligned}
$$

Por hipótese, $D(A)=0$ quando $A$ tem duas linhas consecutivas iguais, então

$$
D\left(\alpha_{1}, \cdots, \alpha_{i}+\beta_{i}, \alpha_{i}+\beta_{i}, \cdots, \alpha_{n}\right)=0
$$

e

$$
D\left(\alpha_{1}, \cdots, \alpha_{i}, \alpha_{i}, \cdots, \alpha_{n}\right)=D\left(\alpha_{1}, \cdots, \beta_{i}, \beta_{i}, \cdots, \alpha_{n}\right)=0 .
$$

Com isso concluímos que $D\left(\alpha_{1}, \cdots, \alpha_{i}, \beta_{i}, \cdots, \alpha_{n}\right)=-D\left(\alpha_{1}, \cdots, \beta_{i}, \alpha_{i}, \cdots, \alpha_{n}\right)$, ou seja, se $A^{\prime}$ é uma matriz obtida permutando duas linhas consecutivas de $A$, então $D\left(A^{\prime}\right)=-D(A)$.

Agora precisamos mostrar que o resultado vale mesmo que as linhas iguais não sejam consecutivas. Suponha que a matriz $B$ seja obtida permutando-se as linhas $i$ e $j$, não necessariamente consecutivas da matriz $A$, tal que $i<j$. Note que podemos obter a matriz $B$ por uma sucessão de permutações de linhas consecutivas de $A$.

Para visualizarmos melhor, vamos transformar a matriz $A$ na matriz $B$ por meio de uma sucessão de permutações de linhas consecutivas e feito isso vamos contar quantas permutações foram necessárias. Temos

$$
\begin{aligned}
& A=\left(\alpha_{1}, \cdots, \alpha_{i-1}, \alpha_{i}, \alpha_{i+1}, \cdots, \alpha_{j-1}, \alpha_{j}, \alpha_{j+1}, \cdots, \alpha_{n}\right) \\
& \mathrm{e} \\
& B=\left(\alpha_{1}, \cdots, \alpha_{i-1}, \alpha_{j}, \alpha_{i+1}, \cdots, \alpha_{j-1}, \alpha_{i}, \alpha_{j+1}, \cdots, \alpha_{n}\right)
\end{aligned}
$$

Começando com a matriz $A$, primeiramente permutamos a linha $\alpha_{i} \operatorname{com}$ a linha $\alpha_{i+1}$, em seguida permutamos a linha $\alpha_{i}$ com a linha $\alpha_{i+2}$ e repetimos assim o procedimento até que consigamos permutar a linha $\alpha_{i}$ com a linha $\alpha_{j}$. Dessa forma, após $k=j-i$ permutações de linhas consecutivas, obtemos a matriz

$$
\left(\alpha_{1}, \cdots, \alpha_{i-1}, \alpha_{i+1}, \cdots, \alpha_{j-1}, \alpha_{j}, \alpha_{i}, \alpha_{j+1}, \cdots, \alpha_{n}\right)
$$

Agora permutamos a linha $\alpha_{j}$ com a linha $\alpha_{j-1}$, em seguida a linha $\alpha_{j}$ com a linha $\alpha_{j-2}$ e repetimos o procedimento até que consigamos permutar a linha $\alpha_{j}$ com a linha $\alpha_{i+1}$. Dessa forma após $k-1$ permutações de linhas consecutivas obtemos a matriz 


$$
B=\left(\alpha_{1}, \cdots, \alpha_{i-1}, \alpha_{j}, \alpha_{i+1}, \cdots, \alpha_{j-1}, \alpha_{i}, \alpha_{j+1}, \cdots, \alpha_{n}\right)
$$

Contando o número de permutações de linhas consecutivas que foram necessárias para a partir da matriz $A$ obtermos a matriz $B$ chegamos ao número de $2 k-1$ permutações. Como a cada permutação de linhas consecutivas o sinal da função $D$ muda em relação a matriz anterior, podemos afirmar que $D(B)=(-1)^{2 k-1} \cdot D(A)$, e como $2 k-1$ é um número impar, concluímos que $D(B)=-D(A)$, como queríamos.

ii) Suponha que $A=\left(a_{i j}\right)_{n \times n}$ é uma matriz qualquer com duas linhas iguais. Sejam essas linhas $\alpha_{i}$ e $\alpha_{j}$ onde $\alpha_{i}=\alpha_{j}$ tal que $i<j$.

Se $j=i+1$, a matriz $A$ tem duas linhas consecutivas iguais e por hipótese $D(A)=0$. além disso, como vimos em (i) essa relação também implica que $D\left(A^{\prime}\right)=-D(A)$ sempre que $A^{\prime}$ for obtida pela permutação de linhas consecutivas de $A$.

Se $j>i+1$, podemos permutar a linha $\alpha_{j}$ com a linha $\alpha_{i+1}$ obtendo uma matriz $B$ que possui duas linhas consecutivas iguais o que implica que $D(B)=0$. Além disso, vimos que quando fazemos essa permutação encontramos $D(B)=-D(A)$, mas como $D(B)=0$, temos que $D(A)=0$. Dessa forma concluímos que a função $D$ descrita neste lema é alternada.

Definição 4.4. Seja $n>1$ um inteiro e seja $A=\left(a_{i j}\right)_{n \times n}$ uma matriz qualquer de ordem $n$. Se $A^{\prime}$ é a matriz quadrada de ordem $n-1$ obtida pela eliminação da linha $i$ e coluna $j$ de $A$ e se $D$ é uma função $(n-1)$-linear sobre as matrizes de ordem $n-1$, definimos $D_{i j}(A)$ como o menor complementar do elemento $a_{i j}$ e expressamos $D_{i j}(A)=D\left(A^{\prime}\right)$.

Teorema 4.1. Seja $n>1$ um inteiro, seja $A=\left(a_{i j}\right)_{n \times n}$ uma matriz qualquer de ordem $n$ e seja $D$ uma função $(n-1)$-linear alternada sobre as matrizes de ordem $n-1$. Para cada $j$ tal que $j \in\{1,2, \cdots, n\}$, a função $E_{j}$ definida por

$$
E_{j}(A)=\sum_{i=1}^{n}(-1)^{i+j} \cdot a_{i j} \cdot D_{i j}(A)
$$

é uma função $n$-linear alternada sobre as matrizes de ordem $n$. Além disso, se $D$ é uma função determinante então $E_{j}$ também será. 
Demonstração. Como $A=\left(a_{i j}\right)_{n \times n}$ é uma matriz de ordem $n$ e $D_{i j}(A)$ é uma função $(n-1)$-linear aplicada a matriz $A^{\prime}=\left(a_{i j}^{\prime}\right)$ de ordem $(n-1)$, obtida pela eliminação da linha $i$ e da coluna $j$ da matriz $A$, concluímos que $D_{i j}(A)$ independe da $i$-ésima linha de $A$. Como $D$ é uma função $(n-1)$-linear que se aplica a qualquer linha exceto a linha $i$, concluimos que $a_{i j} \cdot D_{i j}(A)$ é uma função $n$-linear de $A$. Como vimos no Lema 4.1, uma combinação linear de funções $n$-lineares é $n$-linear, $\operatorname{logo} E_{j}$ é $n$-linear.

Precisamos agora mostrar que $E_{j}$ é uma função alternada. Para isso, basta mostrarmos que $E_{j}(A)=0$ sempre que $A$ tiver duas linhas consecutivas iguais como vimos no Lema 4.4 .

Vamos supor que a matriz $A=\left(\alpha_{1}, \alpha_{2}, \cdots, \alpha_{n}\right)$ tenha duas linhas consecutivas iguais, digamos $\alpha_{k}=\alpha_{k+1}$. Se $i \neq k$ e $i \neq k+1$, a função $D_{i j}(A)$ será aplicada a matriz $A^{\prime}=\left(a_{i j}^{\prime}\right)_{(n-1) \times(n-1)}$ que foi obtida sem eliminar nem a linha $\alpha_{k}$ e nem a linha $\alpha_{k+1}$, logo a matriz $A^{\prime}$ terá duas linhas consecutivas iguais e como por hipótese a função $D$ é alternada, concluímos que $D_{i j}(A)=0$ sempre que $i \neq k$ e $i \neq k+1$. Com isso, perceba que $E_{j}(A)$ pode ser obtido calculando-se o somatório apenas na linha $\alpha_{k}$ e na linha $\alpha_{k+1}$, desse modo temos

$$
E_{j}(A)=(-1)^{k+j} \cdot a_{k j} \cdot D_{k j}(A)+(-1)^{k+1+j} \cdot a_{(k+1) j} \cdot D_{(k+1) j}(A) .
$$

Como $\alpha_{k}=\alpha_{k+1}$, sabemos que $a_{k j}=a_{(k+1) j}$ e $D_{k j}(A)=D_{(k+1) j}(A)$, logo concluímos que a expressão acima é formada pela soma de duas parcelas iguais em módulo, mas que diferem pelo sinal, ou seja, $E_{j}(A)=0$.

A fim de demonstrar a segunda parte do teorema, vamos supor agora que $D$ seja uma função determinante. Se $I_{n}$ é a matriz identidade de ordem $n$, e se $I_{n}^{\prime}=\left(a_{i j}^{\prime}\right)$ é a matriz de ordem $(n-1)$ obtida pela eliminação da linha $i$ e pela coluna $j$ de $I_{n}$, observamos que $I_{n}^{\prime}$ é também a matriz identidade de ordem $n-1$, ou seja, $I_{n}^{\prime}=I_{n-1}$. Como $D$ por hipótese é uma função determinante, e $I_{n}^{\prime}$ é a matriz identidade de ordem $(n-1)$, temos que $D\left(I_{n}^{\prime}\right)=1$.

Decorre da função $E_{j}$ que

$$
E_{j}\left(I_{n}\right)=\sum_{i=1}^{n}(-1)^{i+j} \cdot a_{i j} \cdot D_{i j}\left(I_{n}^{\prime}\right)
$$

Como $I_{n}$ é a matriz identidade de ordem $n$, temos que o elemento $a_{i j}=1$ se o elemento faz parte da diagonal principal da matriz, ou seja, quando $i=j$ e que $a_{i j}=0$ caso contrário. Sendo assim, para calcular $E_{j}\left(I_{n}\right)$, basta calcular o valor para o único elemento da coluna $j$ que é igual a 1 , quando $i=j$. Dessa forma temos que 
$E_{j}\left(I_{n}\right)=(-1)^{i+i} \cdot 1 \cdot 1$ o que implica que $E_{j}\left(I_{n}\right)=1$ e por conseguinte que a função $E_{j}$ é uma função determinante.

Definição 4.5. Seja $n$ um inteiro positivo, seja $A=\left(a_{i j}\right)_{n \times n}$ uma matriz quadrada qualquer de ordem $n$ e seja $D_{i j}$ a função menor complementar relacionada ao elemento $a_{i j}$. Definimos como cofator do elemento $a_{i j}$ e representamos por $A_{i j}$ a função

$$
A_{i j}=(-1)^{i+j} \cdot D_{i j}
$$

A partir dessa definição, podemos reescrever a função determinante $E_{j}$ como

$$
E_{j}(A)=\sum_{i=1}^{n} a_{i j} \cdot A_{i j}(A)
$$

Corolário 4.1. Seja $n$ um inteiro positivo. Existe pelo menos uma função determinante sobre as matrizes quadradas de ordem $n$.

Demonstração. Ja vimos anteriormente nesta mesma seção que existe uma única função determinante sobre as matrizes quadradas de ordem 2. Vimos no Teorema 4.1 que a função $E_{j}$ pode ser usada para produzir uma função determinante sobre as matrizes de ordem $n$ a partir de uma função determinante sobre as matrizes de ordem $n-1$. Dessa forma, podemos utilizar a função determinante sobre as matrizes de ordem 2 que vimos no Exemplo 4.3 para construir uma função determinante sobre as matrizes de ordem 3. Analogamente podemos usar a função determinante sobre as matrizes de ordem 3 que acaba de ser construída para obter uma função determinante sobre as matrizes de ordem 4 .

Podemos repetir esse procedimento indefinidamente obtendo assim uma função determinante aplicada a matrizes de qualquer ordem $n$.

Exemplo 4.5. Seja a matriz $B=\left(b_{i j}\right)_{2 \times 2}$ e a função determinante $D$ sobre as matrizes de ordem 2 , que já vimos ser única e definida por $D(B)=b_{11} b_{22}-b_{12} b_{21}$. Seja ainda a matriz $A=\left(a_{i j}\right)_{3 \times 3}$. Podemos obter uma função determinante sobre a matriz $A$ de ordem 3 a partir da função determinante $D$ utilizando a equação $E_{j}$ vista no teorema 4.1. Dessa forma temos

$$
\begin{aligned}
& E_{1}(A)=a_{11} D\left(\begin{array}{ll}
a_{22} & a_{23} \\
a_{32} & a_{33}
\end{array}\right)-a_{21} D\left(\begin{array}{cc}
a_{12} & a_{13} \\
a_{32} & a_{33}
\end{array}\right)+a_{31} D\left(\begin{array}{cc}
a_{12} & a_{13} \\
a_{22} & a_{23}
\end{array}\right) \\
& E_{1}(A)=a_{11}\left[a_{22} a_{33}-a_{32} a_{23}\right]-a_{21}\left[a_{12} a_{33}-a_{32} a_{13}\right]+a_{31}\left[a_{12} a_{23}-a_{22} a_{13}\right]
\end{aligned}
$$




$$
\begin{aligned}
& E_{1}(A)=a_{11} a_{22} a_{33}-a_{11} a_{32} a_{23}-a_{21} a_{12} a_{33}+a_{21} a_{32} a_{31}+a_{31} a_{12} a_{23}-a_{31} a_{22} a_{13} \\
& E_{2}(A)=-a_{12} D\left(\begin{array}{cc}
a_{21} & a_{23} \\
a_{31} & a_{33}
\end{array}\right)+a_{22} D\left(\begin{array}{cc}
a_{11} & a_{13} \\
a_{31} & a_{33}
\end{array}\right)-a_{32} D\left(\begin{array}{cc}
a_{11} & a_{13} \\
a_{21} & a_{23}
\end{array}\right) \\
& E_{2}(A)=-a_{12}\left[a_{21} a_{33}-a_{31} a_{23}\right]+a_{22}\left[a_{11} a_{33}-a_{31} a_{13}\right]-a_{32}\left[a_{11} a_{23}-a_{21} a_{13}\right] \\
& E_{2}(A)=-a_{12} a_{21} a_{33}+a_{12} a_{31} a_{23}+a_{22} a_{11} a_{33}-a_{22} a_{31} a_{13}-a_{32} a_{11} a_{23}+a_{32} a_{21} a_{13} \\
& E_{3}(A)=a_{13} D\left(\begin{array}{cc}
a_{21} & a_{22} \\
a_{31} & a_{32}
\end{array}\right)-a_{23} D\left(\begin{array}{cc}
a_{11} & a_{12} \\
a_{31} & a_{32}
\end{array}\right)+a_{33} D\left(\begin{array}{cc}
a_{11} & a_{12} \\
a_{21} & a_{22}
\end{array}\right) \\
& E_{3}(A)=a_{13}\left[a_{21} a_{32}-a_{31} a_{22}\right]-a_{23}\left[a_{11} a_{32}-a_{31} a_{12}\right]+a_{33}\left[a_{11} a_{22}-a_{21} a_{12}\right] \\
& E_{3}(A)=a_{13} a_{21} a_{32}-a_{13} a_{31} a_{22}-a_{23} a_{11} a_{32}+a_{23} a_{31} a_{12}+a_{33} a_{11} a_{22}-a_{33} a_{21} a_{12}
\end{aligned}
$$

Como obtivemos as funções $E_{1}(A), E_{2}(A)$ e $E_{3}(A)$ a partir da função determinante $D$ utilizando a equação $E_{j}$, concluímos que as três são funções determinantes sobre as matrizes de ordem 3 . Note que $E_{1}(A)=E_{2}(A)=E_{3}(A)$ e que as três funções possuem a mesma expressão vista na Seção 2 como "Regra de Sarrus", isso parece ser um indício de que as funções determinantes sobre uma matriz de ordem $n$ são únicas. Na próxima seção, nos dedicaremos a provar essa ideia.

Notação 1. Cabe salientar que a função determinante $D$, aplicada a uma matriz $A$ qualquer de ordem $n$ é comumente representada por meio da notação $|A|$.

Assim dada a matriz $A=\left(\begin{array}{cc}a_{11} & a_{12} \\ a_{21} & a_{22}\end{array}\right)$, podemos representar a função Determinante $D$ aplicada a essa matriz por meio da notação $D(A)=|A|=\left|\begin{array}{cc}a_{11} & a_{12} \\ a_{21} & a_{22}\end{array}\right|$.

\subsection{Unicidade da Função Determinante}

Mostramos a seguir que a função determinante $D$, sobre as matrizes de ordem $n$, é única. Tal demonstração dependerá de uma serie de combinações relacionadas aos elementos da matriz unida às ideias já consolidadas anteriormente na Seção 4.1.

Primeiramente, vamos escrever de forma somatorial uma linha qualquer de uma matriz arbitrária. Isso será de grande utilidade nas demonstrações que faremos nesta 
seção e nos possibilitará analisar melhor as diversas combinações que podem ser feitas entre os elementos da matriz.

Seja $D$ uma função $n$-linear alternada sobre as matrizes de ordem $n$. Seja também $A=\left(a_{i j}\right)_{n \times n}$ uma matriz qualquer de ordem $n$. Seja ainda $\alpha_{1}, \alpha_{2}, \cdots, \alpha_{n}$ as linhas da matriz $A$.

Se indicarmos as linhas da matriz identidade de ordem $n$, denominada $I_{n}$, por $\epsilon_{1}, \epsilon_{2}, \cdots, \epsilon_{n}$, então podemos escrever por exemplo

$$
\begin{aligned}
& \alpha_{1}=a_{11} \epsilon_{1}+a_{12} \epsilon_{2}+\cdots+a_{1 n} \epsilon_{n} \\
& \alpha_{2}=a_{21} \epsilon_{1}+a_{22} \epsilon_{2}+\cdots+a_{2 n} \epsilon_{n}
\end{aligned}
$$

De forma análoga, podemos generalizar uma linha qualquer da matriz $A$ por meio da equação:

$$
\alpha_{i}=\sum_{j=1}^{n} a_{i j} \epsilon_{j} \quad \text { onde, } i \in\{1,2, \cdots, n\}
$$

Já que a função $D$ é $n$-linear, podemos escrever $D(A)$ como uma função das linhas da matriz $A$, logo temos $D(A)=D\left(\alpha_{1}, \alpha_{2}, \cdots, \alpha_{n}\right)$. Substituindo o somatório representativo da linha $\alpha_{1}$ na função $D$, temos

$$
\begin{aligned}
& D(A)=D\left(\sum_{j=1}^{n} a_{1 j} \epsilon_{j}, \alpha_{2}, \cdots, \alpha_{n}\right) \\
& D(A)=\sum_{j=1}^{n} a_{1 j} D\left(\epsilon_{j}, \alpha_{2}, \cdots, \alpha_{n}\right)
\end{aligned}
$$

Escrevendo agora a segunda linha como uma somatório, temos

$$
\sum_{p=1}^{n} a_{2 p} \epsilon_{p}
$$

e substituindo na função $D$, temos

$$
D\left(\epsilon_{j}, \alpha_{2}, \cdots, \alpha_{n}\right)=\sum_{p=1}^{n} a_{2 p} D\left(\epsilon_{j}, \epsilon_{p}, \cdots, \alpha_{n}\right)
$$

Repetindo o procedimento empregado nas duas primeiras linhas para as $n$ linhas da matriz $A$ chegaremos a expressão

$$
D(A)=\sum_{k_{1}, k_{2}, \cdots, k_{n}} a_{1 k_{1}} \cdot a_{2 k_{2}} \cdot a_{3 k_{3}} \cdots a_{n k_{n}} D\left(\epsilon_{k_{1}}, \epsilon_{k_{2}}, \cdots, \epsilon_{k_{n}}\right)
$$


Note que o somatório percorre todas as sequências $\left(k_{1}, k_{2}, \cdots, k_{n}\right)$ em que $k_{i} \in$ $\{1,2, \cdots, n\}$. Note também que a função $D$ é uma soma finita de funções do tipo visto no Exemplo 4.1 e que a Equação (4.9) foi desenvolvida unicamente com a hipótese de que $D$ é uma função $n$-linear. Um caso particular da função $D$ foi visto no Exemplo 4.2 descrito na Equação (4.3).

Como $D$ é uma função alternada, $D\left(\epsilon_{k 1}, \epsilon_{k 2}, \cdots, \epsilon_{k n}\right)=0$, sempre que dois índices $k_{i}$ forem iguais. Caso contrário, ou seja, sempre que a sequencia $k_{1}, k_{2}, \cdots, k_{n}$ de índices não tiver dois $k_{i}$ iguais, $D\left(\epsilon_{k 1}, \epsilon_{k 2}, \cdots, \epsilon_{k n}\right) \neq 0$. A todas as combinações possíveis que não possuem dois índices $k_{i}$ iguais, damos o nome de permutações de grau $n$, em outras palavras, podemos dizer que uma permutação de grau $n$ é uma função bijetiva do conjunto $\{1,2, \cdots, n\}$ nele mesmo. Denominando essa função bijetiva por $\sigma$, podemos dizer que $\sigma$ associa o conjunto $\{1,2, \cdots, n\}$ ao conjunto $\left\{\sigma_{1}, \sigma_{2}, \cdots, \sigma_{n}\right\}$. Note que essa função é apenas uma forma de generalizar todas a permutações de grau $n$ possíveis para os índices das linhas da matriz identidade de ordem $n$. Utilizando a função $\sigma$ para organizar os índices da matriz $\left(\epsilon_{\sigma_{1}}, \epsilon_{\sigma_{2}}, \cdots, \epsilon_{\sigma_{n}}\right)$, podemos reescrever a função $D$ por meio da equação

$$
D(A)=\sum_{\sigma} a_{1 \sigma_{1}} \cdot a_{2 \sigma_{2}} \cdot a_{3 \sigma_{3}} \cdots a_{n \sigma_{n}} D\left(\epsilon_{\sigma_{1}}, \epsilon_{\sigma_{2}}, \cdots, \epsilon_{\sigma_{n}}\right),
$$

onde $\sigma$ percorre todas as permutações de grau $n$.

Mostraremos a seguir que $D\left(\epsilon_{\sigma_{1}}, \epsilon_{\sigma_{2}}, \cdots, \epsilon_{\sigma_{n}}\right)= \pm D\left(\epsilon_{1}, \epsilon_{2}, \cdots, \epsilon_{n}\right)$ e que o sinal depende unicamente da permutação $\sigma$ que foi feita.

Primeiramente, perceba que a sequência $\left(\sigma_{1}, \sigma_{2}, \cdots, \sigma_{n}\right)$ pode ser obtida a partir da sequencia $(1,2, \cdots, n)$ por meio de um número finito $m$ de trocas de pares de elementos. Se por exemplo, $\sigma_{1} \neq 1$, podemos trocar $\sigma_{1}$ e 1 obtendo a sequencia $\left(\sigma_{1}, \cdots, 1, \cdots\right)$. Repetindo esse procedimento quantas vezes forem necessárias, podemos obter a sequencia $\left(\sigma_{1}, \sigma_{2}, \cdots, \sigma_{n}\right)$ após $m$ trocas de pares de índices, onde $m \leq n$.

Como, por hipótese, a função $D$ é alternada, sabemos que a cada vez que trocamos duas linhas quaisquer da matriz, o sinal da função $D$ muda. Dessa forma, se passamos da sequencia $(1,2, \cdots, n)$ para a sequencia de índices $\left(\sigma_{1}, \sigma_{2}, \cdots, \sigma_{n}\right)$ por meio de $m$ trocas, podemos escrever que $D\left(\epsilon_{\sigma_{1}}, \epsilon_{\sigma_{2}}, \cdots, \epsilon_{\sigma_{n}}\right)=(-1)^{m} D\left(\epsilon_{1}, \epsilon_{2}, \cdots, \epsilon_{n}\right)$. Em particular, se $D$ é uma função determinante, temos que $D\left(\epsilon_{1}, \epsilon_{2}, \cdots, \epsilon_{n}\right)=1$, logo podemos escrever que

$$
D\left(\epsilon_{\sigma_{1}}, \epsilon_{\sigma_{2}}, \cdots, \epsilon_{\sigma_{n}}\right)=(-1)^{m}
$$


onde $m$, depende unicamente da permutação $\sigma$. Assim, podemos afirmar que se $D$ é uma função determinante, então $D$ associa sempre o mesmo valor a matriz $\left(\epsilon_{\sigma_{1}}, \epsilon_{\sigma_{2}}, \cdots, \epsilon_{\sigma_{n}}\right)$, a saber, 1 ou -1 .

Como $m$ é o número de trocas de índices necessários, sabemos que $m$ é inteiro e positivo, logo $m$ só pode ser par ou impar. Caso $m$ seja par, dizemos que a permutação $\sigma$ é par, e então $(-1)^{m}=1$ e caso $m$ seja impar, dizemos que a permutação $\sigma$ é impar, e então $(-1)^{m}=-1$. Dessa maneira, podemos definir o sinal da permutação $\sigma$, por meio da relação

$$
\text { sinal } \sigma=\left\{\begin{array}{c}
1, \text { se } \sigma \text { é par } \\
-1, \text { se } \sigma \text { é impar }
\end{array}\right.
$$

Com isso, temos que a função $D$ aplicada a matriz obtida pela permutação de grau $n$ das linhas da matriz identidade $I_{n}$ é dada por

$$
D\left(\epsilon_{\sigma_{1}}, \epsilon_{\sigma_{2}}, \cdots, \epsilon_{\sigma_{n}}\right)=(\operatorname{sinal} \sigma) D\left(\epsilon_{1}, \epsilon_{2}, \cdots, \epsilon_{n}\right)
$$

Substituindo essa relação na equação (4.10), chegamos a uma nova equação da função $D$ representada por

$$
D(A)=\sum_{\sigma}\left[(\operatorname{sinal} \sigma) \cdot a_{1 \sigma_{1}} \cdot a_{2 \sigma_{2}} \cdot a_{3 \sigma_{3}} \cdots a_{n \sigma_{n}}\right] \cdot D\left(I_{n}\right)
$$

Note que essa equação representa uma relação única e fechada para a função $D$, $n$-linear e alternada que só varia em função de $D\left(I_{n}\right)$. Suponha por hipótese que $D$ é uma função determinante, logo temos que $D\left(I_{n}\right)=1$, e com isso, chegamos a uma fórmula única que expressa a função determinante $D$, denominada a partir de agora por det, descrita por

$$
D(A)=\operatorname{det}(A)=\sum_{\sigma}\left[(\operatorname{sinal} \sigma) \cdot a_{1 \sigma_{1}} \cdot a_{2 \sigma_{2}} \cdot a_{3 \sigma_{3}} \cdots a_{n \sigma_{n}}\right] .
$$

Podemos resumir a demonstração desenvolvida até aqui sobre a unicidade da função determinante $D$ aplicada as matrizes de ordem $n$ por meio do teorema abaixo.

Teorema 4.2. Seja $n$ um inteiro positivo. Existe uma única função determinante $D$ sobre as matrizes $A=\left(a_{i j}\right)_{n \times n}$, que a partir de agora denominaremos função det, definida pela equação (4.13). Se $D$ é uma função $n$-linear alternada qualquer aplicada as matrizes $A=\left(a_{i j}\right)$ de ordem $n$, então 


$$
D(A)=\operatorname{det}(A) \cdot D\left(I_{n}\right)
$$

Já que demonstramos a unicidade da função determinante, que era o objetivo dessa seção, iremos, a seguir, nos dedicar a fazer algumas observações finais sobre as permutações de grau $n$.

Primeiramente note que existem exatamente $n !=1 \cdot 2 \cdot 3 \cdots n$ permutações de grau $n$. Tal fato pode ser deduzido do princípio fundamental da contagem, pois se $\sigma$ é uma permutação qualquer de grau $n$, então existem $n$ possibilidades para $\sigma_{1}$, $(n-1)$ possibilidades para $\sigma_{2},(n-2)$ possibilidades para $\sigma_{3}$, e assim por diante até que teremos 2 possibilidades para $\sigma_{n-1}$ e 1 possibilidade para $\sigma_{n}$. Dessa forma, pelo princípio fundamental da contagem, o número de permutações de grau $n$ é dado por

$$
n(n-1)(n-2) \cdots 2 \cdot 1=n !
$$

Perceba na análise da equação (4.13), que $\operatorname{det}(A)$ é uma soma de $n$ ! termos, cada um representado por uma permutação distinta de grau $n$.

Um termo genérico desse somatório, é o produto $a_{1 \sigma_{1}} \cdot a_{2 \sigma_{2}} \cdots a_{n \sigma_{n}}$, que é o produto de $n$ elementos distintos da matriz $A$, sendo um único elemento de cada linha e um de cada coluna envolvidos nesse produto. O sinal desse termo será dado pelo sinal da função $\sigma$, sendo positivo quando $\sigma$ for par e negativo quando $\sigma$ for impar.

Sejam $\sigma$ e $\tau$ duas permutações quaisquer de grau $n$. Podemos definir o produto das permutações $\sigma$ por $\tau$ como uma composição de funções onde

$$
(\sigma \tau)(i)=\sigma(\tau(i))
$$

Digamos que $\epsilon$ seja a permutação idêntica, ou seja, $\epsilon(i)=i$, isso significa que a permutação $\epsilon$ origina a própria matriz identidade de ordem $n$ denominada $I_{n}$. Cada permutação $\sigma$, possui uma permutação inversa $\sigma^{-1}$, onde

$$
\sigma \sigma^{-1}=\sigma^{-1} \sigma=\epsilon
$$

A propriedade fundamental do produto de permutações de grau $n$, nos diz que, sendo $\sigma$ e $\tau$ duas permutações quaisquer de grau $n$, temos

$$
\operatorname{sinal}(\sigma \tau)=(\operatorname{sinal} \sigma) \cdot(\operatorname{sinal} \tau)
$$


Observe que a demonstração da propriedade fundamental do produto de permutações, descrita na Equação (4.14), pode ser realizada por meio de uma propriedade fundamental dos determinantes, como veremos a seguir.

Sejam $\sigma$ e $\tau$ duas permutações quaisquer de grau $n$, sejam $\epsilon_{1}, \epsilon_{2}, \cdots, \epsilon_{n}$ as linhas da matriz identidade de ordem $n$ e sejam $A=\left(\epsilon_{\tau_{1}}, \epsilon_{\tau_{2}}, \cdots, \epsilon_{\tau_{n}}\right)$ e $B=\left(\epsilon_{\sigma_{1}}, \epsilon_{\sigma_{2}}, \cdots, \epsilon_{\sigma_{n}}\right)$, duas matrizes obtidas por permutações das linhas da matriz identidade de ordem $n$. Observe que a $i$-ésima linha da matriz $A$ contém exatamente um elemento não nulo, seu valor é 1 e fica localizado na coluna $\tau_{i}$. Note também que $\epsilon_{\sigma \tau_{i}}$ é a $i$-ésima linha da matriz produto $A B$. Logo, $\operatorname{como} \operatorname{det}(A)=\operatorname{sinal} \tau, \operatorname{det}(B)=\operatorname{sinal} \sigma$, e $\operatorname{det}(A B)=\operatorname{sinal}(\sigma \tau)$, desde que possamos provar o teorema 4.3, concluímos que

$$
\operatorname{sinal}(\sigma \tau)=(\operatorname{sinal} \sigma) \cdot(\operatorname{sinal} \tau) .
$$

O teorema enunciado abaixo é conhecido como Teorema de Binet.

Teorema 4.3. Seja $n$ um inteiro positivo e sejam $A=\left(a_{i j}\right)_{n \times n}$ e $B=\left(b_{i j}\right)_{n \times n}$ duas matrizes de ordem $n$. Então temos

$$
\operatorname{det}(A B)=(\operatorname{det} A)(\operatorname{det} B)
$$

Demonstração. Sejam $A=\left(a_{i j}\right)_{n \times n}$ e $B=\left(b_{i j}\right)_{n \times n}$ duas matrizes de ordem $n$, e considere a função $D$ definida por $D(A)=\operatorname{det}(A B)$.

Se indicarmos as linhas da matriz $A$ por $\alpha_{1}, \alpha_{2}, \cdots, \alpha_{n}$, temos que as linhas da matriz $A B$ são dadas por $\alpha_{1} B, \alpha_{2} B, \cdots, \alpha_{n} B$. Assim podemos reescrever $D$ como

$$
D\left(\alpha_{1}, \alpha_{2}, \cdots, \alpha_{n}\right)=\operatorname{det}\left(\alpha_{1} B, \alpha_{2} B, \cdots, \alpha_{n} B\right)
$$

Perceba que $\alpha_{j} B$ indica o produto da matriz $\alpha_{j}$ de ordem $(1 \times n)$, matriz linha, pela matriz $B$ de ordem $n$. Como $\left(c \alpha_{i}+\alpha_{i}^{\prime}\right) B=c \alpha_{i} B+\alpha_{i}^{\prime} B$ e como det é $n$-linear, concluímos que $D$ é $n$-linear. Se $\alpha_{i}=\alpha_{j}$, então $\alpha_{i} B=\alpha_{j} B$, e como det é alternada, $D\left(\alpha_{1}, \alpha_{2}, \cdots, \alpha_{n}\right)=0$, logo concluímos que $D$ é alternada.

Como $D$ é uma função $n$-linear alternada, pelo Teorema 4.2 podemos escrever que $D(A)=(\operatorname{det} A) D(I)$, mas por hipótese $D(I)=\operatorname{det}(I B)=\operatorname{det} B$, portanto concluímos que

$$
\operatorname{det}(A B)=D(A)=(\operatorname{det} A) \cdot(\operatorname{det} B) .
$$




\subsection{Propriedades dos Determinantes}

Iremos mostrar nesta seção algumas propriedades importantes relacionadas a função determinante sobre as matrizes de ordem $n$. Verificamos no decorrer que essas propriedades podem facilitar o trabalho com os Determinantes.

Propriedade 4.1. Seja $n$ um inteiro tal que $n \geq 2$ e seja $M$ uma matriz quadrada de ordem $n$. Se $M^{-1}$ é a matriz inversa da matriz $M$, então $\operatorname{det}\left(M^{-1}\right)=\frac{1}{\operatorname{det}(M)}$.

Para demostrar essa propriedade, iremos utilizar de forma direta o Teorema de Binet.

Se $M^{-1}$ é a inversa de $M$, então vale a relação $M \cdot M^{-1}=I_{n}$. Com isso temos que $\operatorname{det}\left(M \cdot M^{-1}\right)=\operatorname{det}\left(I_{n}\right)=1$

Utilizando Binet, temos $\operatorname{det}(M) \cdot \operatorname{det}\left(M^{-1}\right)=1$ o que implica que

$$
\operatorname{det}\left(M^{-1}\right)=\frac{1}{\operatorname{det}(M)}
$$

Perceba que essa propriedade denota que $M$ só terá inversa se $\operatorname{det}(M) \neq 0$

Propriedade 4.2. Se $n$ é um inteiro positivo, então uma função determinante sobre as matrizes de ordem $n$ é também $n$-linear em relação as colunas da matriz.

Para mostrar isso, basta provar que sendo $A^{t}$ a matriz transposta de $A$, vale a relação

$$
\operatorname{det}\left(A^{t}\right)=\operatorname{det}(A)
$$

Se $A=\left(a_{i j}\right)$ é uma matriz qualquer de ordem $n, A^{t}$ é a matriz transposta de $A$ e $\sigma$ é uma permutação de grau $n$ das linhas da matriz identidade de ordem $n$, então

$$
a_{i \sigma_{i}}^{t}=a_{\sigma_{i} i}
$$

Utilizando a Equação (4.13), e a relação acima, chegamos a seguinte função:

$$
\operatorname{det}\left(A^{t}\right)=\sum_{\sigma}(\operatorname{sinal} \sigma) a_{\sigma_{1} 1} \cdot a_{\sigma_{2} 2} \cdots a_{\sigma_{n} n}
$$

Se $\sigma_{i}=j$, temos que $\sigma^{-1} j=i$, dessa forma, temos que $a_{\sigma_{i} i}=a_{j \sigma^{-1} j}$, com isso concluímos que

$$
a_{\sigma_{1} 1} \cdot a_{\sigma_{2} 2} \cdots a_{\sigma_{n} n}=a_{1 \sigma^{-1} 1} \cdot a_{2 \sigma^{-1} 2} \cdots a_{n \sigma^{-1} n} .
$$


Como $\sigma \sigma^{-1}$ é a permutação idêntica, sabemos que $(\operatorname{sinal} \sigma) \cdot\left(\operatorname{sinal} \sigma^{-1}\right)=1$, ou seja, $\operatorname{sinal}\left(\sigma^{-1}\right)=\operatorname{sinal}(\sigma)$. Sabemos também que, quando $\sigma$ percorre todas as permutações de grau $n, \sigma^{-1}$ também percorre, logo concluímos que

$$
\operatorname{det}\left(A^{t}\right)=\sum_{\sigma}\left(\operatorname{sinal} \sigma^{-1}\right) \cdot a_{1 \sigma^{-1} 1} \cdot a_{2 \sigma^{-1} 2} \cdots a_{n \sigma^{-1} n}=\operatorname{det}(A) .
$$

Conhecido como Teorema de Laplace, a propriedade enunciada a seguir pode ser utilizada para calcular o determinante para matrizes de qualquer ordem.

Propriedade 4.3. Seja $n$ um inteiro positivo e seja $A=\left(a_{i j}\right)$ uma matriz qualquer de ordem $n$.

$$
\operatorname{det}(A)=\sum_{i=1}^{n} a_{i j} \cdot A_{i j}=\sum_{j=1}^{n} a_{i j} \cdot A_{i j}
$$

onde $A_{i j}=(-1)^{i+j} \cdot D_{i j}$ é chamado de cofator do elemento $a_{i j}$ e $D_{i j}$ é o determinante da matrizes de ordem $n-1$ obtida eliminando-se a linha $i$ e a coluna $j$ da matriz $A$.

Perceba que este teorema, também chamado de Desenvolvimento de Laplace, utiliza uma linha ou uma coluna qualquer da matriz $A$ para calcular o seu determinante, e como a equação consiste em um somatório de produtos de elementos por cofatores, fica claro que quanto mais elementos nulos tiver a fileira escolhida, mais fácil será o cálculo do determinante.

Já sabemos que este teorema é válido para uma coluna qualquer da matriz, pois a equação enunciada representa a mesma vista na função $E_{j}$ na Equação (4.7). Para demonstrar o teorema em sua totalidade, basta provar que também é valido para uma linha qualquer da matriz $A$.

Vamos supor que a matriz $A$ tenha suas linhas representadas por $\alpha_{1}, \alpha_{2}, \cdots, \alpha_{n}$ e suas colunas representadas por $\beta_{1}, \beta_{2}, \cdots, \beta_{n}$. Dessa forma utilizando a função $E_{j}$, podemos calcular o determinante da matriz $A$ utilizando qualquer uma de suas $\beta_{k}$ colunas. Se trocarmos cada uma das linhas $\alpha_{k}$ pela coluna $\beta_{k}$ correspondente com todos os seus elementos, obteremos uma nova matriz $A^{t}$ que é a matriz transposta da matriz $A$. Dessa forma, as linhas $\alpha_{k}$ em $A$ se transformam nas colunas da matriz $A^{t} \mathrm{e}$ utilizando a mesma função $E_{j}$ podemos calcular o determinante da matriz $A^{t}$ utilizando qualquer uma de suas $\alpha_{k}$ colunas. Como vimos na Propriedade $4.2, \operatorname{det}(A)=\operatorname{det}\left(A^{t}\right)$, logo a função $E_{j}$ pode ser utilizada tanto nas colunas $\beta_{k}$ como nas linhas $\alpha_{k}$ da matriz $A$ para calcular o determinante da matriz $A$, o que demonstra exatamente o enunciado no Desenvolvimento de Laplace. 
Conhecido como Teorema de Jacobi, a propriedade enunciada a seguir é uma facilitadora no cálculo de alguns determinantes.

Propriedade 4.4. Se B é uma matriz obtida a partir de A, somando-se um múltiplo de uma linha da matriz A a outra linha, então o determinante não se altera, ou seja, vale a relação:

$$
\operatorname{det}(B)=\operatorname{det}(A)
$$

Já que a função determinante também é $n$-linear em relação as colunas, após demonstrarmos essa propriedade, ela também será valida em relação as colunas.

Sejam $\alpha_{i}$ e $\alpha_{j}$, duas linhas quaisquer da matriz $A$ de ordem $n$. Seja ainda, $B$ uma matriz obtida a partir da matriz $A$, somando-se $c \alpha_{j}$ a $\alpha_{i}$, onde $i<j$. Com isso temos $A=\left(\alpha_{1}, \alpha_{2}, \cdots, \alpha_{i}, \cdots, \alpha_{j}, \cdots, \alpha_{n}\right)$ e $B=\left(\alpha_{1}, \alpha_{2}, \cdots, \alpha_{i}+c \alpha_{j}, \cdots, \alpha_{j}, \cdots, \alpha_{n}\right)$. Já que a função det é $n$-linear como uma função da $i$-ésima linha, temos

$$
\begin{gathered}
\operatorname{det}(B)=\operatorname{det}\left(\alpha_{1}, \alpha_{2}, \cdots, \alpha_{i}+c \alpha_{j}, \cdots, \alpha_{j}, \cdots, \alpha_{n}\right) \\
\operatorname{det}(B)=\operatorname{det}\left(\alpha_{1}, \alpha_{2}, \cdots, \alpha_{i}, \cdots, \alpha_{j}, \cdots, \alpha_{n}\right)+\operatorname{det}\left(\alpha_{1}, \alpha_{2}, \cdots, \alpha_{j}, \cdots, \alpha_{j}, \cdots, \alpha_{n}\right) \\
\operatorname{det}(B)=\operatorname{det}(A)+c \cdot \operatorname{det}\left(\alpha_{1}, \alpha_{2}, \cdots, \alpha_{j}, \cdots, \alpha_{j}, \cdots, \alpha_{n}\right)
\end{gathered}
$$

Como a função det é alternada, temos que $\operatorname{det}\left(\alpha_{1}, \alpha_{2}, \cdots, \alpha_{j}, \cdots, \alpha_{j}, \cdots, \alpha_{n}\right)=0$, logo concluímos que

$$
\operatorname{det}(B)=\operatorname{det}(A)
$$

Propriedade 4.5. Seja $M$ uma matriz de ordem $n=r+s$ do tipo 


$$
M=\left(\begin{array}{cccc|cccc}
a_{11} & a_{12} & \cdots & a_{1 r} & b_{1, r+1} & b_{1, r+2} & \cdots & b_{1, r+s} \\
a_{21} & a_{22} & \cdots & a_{2 r} & b_{2, r+1} & b_{2, r+2} & \cdots & b_{2, r+s} \\
\vdots & \vdots & \vdots & \vdots & \vdots & \vdots & \vdots & \vdots \\
a_{r 1} & a_{r 2} & \cdots & a_{r r} & b_{r, r+1} & b_{r, r+2} & \cdots & b_{r, r+s} \\
a_{r+1,1} & a_{r+1,2} & \cdots & a_{r+1, r} & c_{r+1, r+1} & c_{r+1, r+2} & \cdots & c_{r+1, r+s} \\
\vdots & \vdots & \vdots & \vdots & \vdots & \vdots & \vdots & \vdots \\
a_{r+s, 1} & a_{r+s, 2} & \cdots & a_{r+s, r} & c_{r+s, r+1} & c_{r+s, r+2} & \cdots & c_{r+s, r+s}
\end{array}\right)
$$

Dizemos que uma tal matriz $M$ é uma matriz formada por blocos de matrizes da seguinte forma:

$$
M=\left(\begin{array}{ll}
A & B \\
0 & C
\end{array}\right)
$$

onde $A=\left(a_{i j}\right)_{r \times r}$ é uma matriz qualquer de ordem $r$, onde $B=\left(b_{i j}\right)_{r \times s}$ é uma matriz qualquer de ordem $r \times s$, onde $C=\left(c_{i j}\right)_{s \times s}$ é uma matriz qualquer de ordem $s \times s$ e 0 é a matriz nula de ordem $s \times r$.

Dessa forma, para qualquer que seja a matriz $M$ formada em blocos como vimos acima, podemos afirmar que

$$
\operatorname{det}(M)=\operatorname{det}(A) \cdot \operatorname{det}(C)
$$

Para demonstrar essa propriedade, primeiramente, vamos definir uma função $D$, tal que

$$
D(A, B, C)=\operatorname{det}\left(\begin{array}{cc}
A & B \\
0 & C
\end{array}\right) .
$$

Fixando $A$ e $B$, temos que a função $D$ é alternada e $s$-linear como uma função das linhas de $C$. Se utilizarmos o Teorema 4.2, sabendo que 0 é a matriz nula de ordem $s \times r$ e que $I_{s}$ é a matriz identidade de ordem $s$, podemos afirmar que

$$
D(A, B, C)=\operatorname{det}(C) \cdot D\left(A, B, I_{s}\right)
$$


Subtraindo das linhas de $B$, múltiplos convenientes da matriz $I_{s}$ e sabendo, como afirma o Teorema 4.2, que o determinante permanece o mesmo quando realizamos esse procedimento, podemos afirmar que

$$
D\left(A, B, I_{s}\right)=D\left(A, 0, I_{s}\right)
$$

Analogamente, se fixarmos a matriz $I_{s}$, podemos afirmar que $D$ é uma função alternada e $r$-linear como uma função das linhas de $A$. Conhecendo o Teorema $4.2 \mathrm{e}$ sendo $I_{r}$ a matriz identidade de ordem $r$, podemos então afirmar que

$$
D\left(A, 0, I_{s}\right)=\operatorname{det}(A) \cdot D\left(I_{r}, 0, I_{s}\right)
$$

Como no início definimos

$$
D(A, B, C)=\operatorname{det}\left(\begin{array}{ll}
A & B \\
0 & C
\end{array}\right)
$$

podemos então afirmar que

$$
D\left(I_{r}, 0, I_{s}\right)=\operatorname{det}\left(\begin{array}{cc}
I_{r} & 0 \\
0 & I_{s}
\end{array}\right) .
$$

Note que a matriz $\left(\begin{array}{cc}I_{r} & 0 \\ 0 & I_{s}\end{array}\right)$ é a matriz identidade de ordem $n=r+s$, logo concluímos que $D\left(I_{r}, 0, I_{s}\right)=1$.

A partir dessas relações podemos afirmar o seguinte:

$$
D(A, B, C)=\operatorname{det}(C) \cdot D\left(A, B, I_{s}\right)=\operatorname{det}(C) \cdot D\left(A, 0, I_{s}\right)=\operatorname{det}(C) \cdot \operatorname{det}(A)
$$

Seguindo um raciocínio análogo e dada a matriz $M$ formada em blocos pelas matrizes $A, B, C$ e pela matriz nula, podemos demonstrar também que

$$
\operatorname{det}\left(\begin{array}{cc}
A & 0 \\
B & C
\end{array}\right)=\operatorname{det}(A) \cdot \operatorname{det}(C)
$$


Exemplo 4.6. Dada a matriz $A$ definida abaixo, vamos utilizar a Propriedade 4.4 para calcular seu determinante.

$$
A=\left(\begin{array}{cccc}
1 & 2 & -1 & 3 \\
-1 & 4 & 1 & 6 \\
2 & -3 & 1 & 1 \\
3 & -2 & 2 & 5
\end{array}\right)
$$

Primeiro vamos pegar a primeira coluna e somar a terceira coluna, obtendo assim a matriz $B$ que pelo Teorema 4.2, terá o mesmo determinante da matriz $A$.

$$
B=\left(\begin{array}{cccc}
1 & 2 & 0 & 3 \\
-1 & 4 & 0 & 6 \\
2 & -3 & 3 & 1 \\
3 & -2 & 5 & 5
\end{array}\right)
$$

Agora vamos multiplicar a segunda coluna por $\frac{-3}{2}$ e somar com a quarta coluna, obtendo a matriz $C$ que pelo teorema 4.2, terá o mesmo determinante da matriz $B$.

$$
C=\left(\begin{array}{cccc}
1 & 2 & 0 & 0 \\
-1 & 4 & 0 & 0 \\
2 & -3 & 3 & \frac{11}{2} \\
3 & -2 & 5 & 8
\end{array}\right)
$$

Note que a matriz $C$ pode ser descrita em blocos como vimos na propriedade 4.4, logo podemos afirmar que

$$
\operatorname{det}(C)=\operatorname{det}\left(\begin{array}{cc}
1 & 2 \\
-1 & 4
\end{array}\right) \cdot \operatorname{det}\left(\begin{array}{cc}
3 & \frac{11}{2} \\
5 & 8
\end{array}\right)=(4+2)\left(24-\frac{55}{2}\right)=-21
$$

Como vimos que $\operatorname{det}(A)=\operatorname{det}(B)=\operatorname{det}(C)$, temos que

$$
\operatorname{det}(A)=-21 .
$$


Propriedade 4.6. Se uma matriz $A$ qualquer de ordem $n$ tiver uma fileira inteira nula, seja uma linha ou uma coluna, então $\operatorname{det}(A)=0$.

Calculando $\operatorname{det}(A)$ por meio da fileira nula através do Desenvolvimento de Laplace, como todos os elementos envolvidos na função somatória são nulos, chegamos a con$\operatorname{clusão~que~} \operatorname{det}(A)=0$.

Propriedade 4.7. Se multiplicarmos uma fileira qualquer de uma matriz $M$ de ordem $n$ por um número $k$, encontraremos uma nova matriz $M^{\prime}$ tal que $\operatorname{det}\left(M^{\prime}\right)=k \cdot \operatorname{det}(M)$.

Como a função det é $n$-linear em relação as linhas e as colunas da matriz $M$, podemos afirmar que sendo $\alpha_{i}$ uma linha ou uma coluna qualquer da matriz $M$, $\operatorname{det}(M)=\operatorname{det}\left(\alpha_{i}\right)$ mantendo as outras fileiras fixas.

Dessa forma temos $\operatorname{det}\left(k \cdot \alpha_{i}+\alpha_{i}^{\prime}\right)=k \cdot \operatorname{det}\left(\alpha_{i}\right)+\operatorname{det}\left(\alpha_{i}^{\prime}\right)$ e fazendo $\alpha_{i}^{\prime}=0$, concluímos que $\operatorname{det}\left(k \cdot \alpha_{i}\right)=k \cdot \operatorname{det}\left(\alpha_{i}\right)=k \cdot \operatorname{det}(M)$.

Propriedade 4.8. Seja $M$ uma matriz de ordem $n \geq 2$ e seja $M^{\prime}$ a matriz obtida pela troca de duas fileiras quaisquer da matriz $M$, então $\operatorname{det}\left(M^{\prime}\right)=-\operatorname{det}(M)$.

Como já vimos anteriormente essa é uma propriedade de toda função alternada, logo já está demonstrada.

Propriedade 4.9. Seja $M$ uma matriz de ordem $n \geq 2$. Se $M$ tem duas fileiras iguais, então $\operatorname{det}(M)=0$.

Como já vimos anteriormente essa é uma propriedade de toda função alternada, logo já está demonstrada.

Propriedade 4.10. Seja $A$ uma matriz qualquer de ordem $n$. A soma dos elementos de uma fileira qualquer de $A$ pelos cofatores dos elementos respectivos em uma fileira paralela é igual a zero. Essa propriedade é conhecida como Teorema de Cauchy.

Para demonstrarmos essa propriedade, basta mostrar que sendo $C_{i j}$ o cofator do elemento $a_{i j}$, sempre que $j \neq k$, temos

$$
\sum_{i=1}^{n} a_{i k} \cdot C_{i j}=0 .
$$

Para isso, vamos supor que a matriz $B$ foi obtida substituindo-se a $j$-ésima coluna da matriz $A$ por sua $k$-ésima coluna. 
Note que a matriz $B$ obtida tem duas colunas iguais o que faz com que $\operatorname{det}(B)=0$. Seja $D_{i j}$ o cofator do elemento $b_{i j}$ da matriz $B$ e seja ainda $C_{i j}$ o cofator do elemento $a_{i j}$ da matriz $A$. Como $D_{i j}$ e $C_{i j}$ são obtidos excluindo a coluna $j$ das matrizes $B$ e $A$ respectivamente, e como a única diferença entre as matrizes $A$ e $B$ é a coluna $j$, temos que as matrizes resultantes após essa exclusão são iguais, logo concluímos que $D_{i j}=C_{i j}$. Alem disso, como a matriz $B$ foi obtida da matriz $A$ substituindo a coluna $j$ pela coluna $k$, concluímos que $b_{i j}=a_{i k}$

Utilizando a função $E_{j}$ do Teorema 4.1 e sabendo que $\operatorname{det}(B)=0$, temos que

$$
\sum_{i=1}^{n} b_{i j} \cdot D_{i j}=0
$$

e assim

$$
\sum_{i=1}^{n} a_{i k} \cdot C_{i j}=0
$$

Propriedade 4.11. Seja $n$ um inteiro tal que $n \geq 2$ e seja $M$ uma matriz quadrada qualquer de ordem $n$. Se $M$ tem duas fileiras paralelas formadas por elemento respectivamente proporcionais, então $\operatorname{det}(M)=0$.

Para demonstrar essa propriedade, vamos supor que a matriz $M$ possui duas linhas proporcionais $\alpha_{i}$ e $\alpha_{j}$ tais que $\alpha_{i}=k \cdot \alpha_{j}$. Dessa forma temos

$$
M=\left(\alpha_{1}, \alpha_{2}, \cdots, \alpha_{i}, \cdots, \alpha_{j}, \cdots, \alpha_{n}\right)=\left(\alpha_{1}, \alpha_{2}, \cdots, k \cdot \alpha_{j}, \cdots, \alpha_{j}, \cdots, \alpha_{n}\right) .
$$

Como a função det é $n$-linear, podemos escrever det como

$$
\operatorname{det}(M)=k \cdot \operatorname{det}\left(\alpha_{1}, \alpha_{2}, \cdots, \cdot \alpha_{j}, \cdots, \alpha_{j}, \cdots, \alpha_{n}\right)
$$

Sabemos que a função det é alternada, $\operatorname{logo} \operatorname{det}(M)=k \cdot 0=0$.

Utilizando um raciocínio análogo, podemos mostrar que a propriedade também vale para as colunas da matriz $M$.

Propriedade 4.12. Conhecida como propriedade da adição de determinantes, enunciamos esta propriedade da seguinte forma. Seja $n$ um inteiro positivo qualquer e seja $M$ uma matriz quadrada de ordem $n$. Se $\left(\alpha_{1}, \alpha_{2}, \cdots, \alpha_{i}, \cdots, \alpha_{n}\right)$ são as linhas da matriz $M$ e se $\alpha_{i}=\beta_{i}+\lambda_{i}$, então $\operatorname{det}(M)=\operatorname{det}(A)+\operatorname{det}(B)$ onde $A$ e $B$ são matrizes quadradas de ordem $n$ tais que $A=\left(\alpha_{1}, \alpha_{2}, \cdots, \beta_{i}, \cdots, \alpha_{n}\right)$ e $B=\left(\alpha_{1}, \alpha_{2}, \cdots, \lambda_{i}, \cdots, \alpha_{n}\right)$.

A demonstração desta propriedade deriva diretamente do fato da função det ser $n$-linear. desta forma temos 


$$
\begin{gathered}
\operatorname{det}(M)=\operatorname{det}\left(\alpha_{1}, \alpha_{2}, \cdots, \beta_{i}+\lambda_{i}, \cdots, \alpha_{n}\right) \\
\operatorname{det}(M)=\operatorname{det}\left(\alpha_{1}, \alpha_{2}, \cdots, \beta_{i}, \cdots, \alpha_{n}\right)+\operatorname{det}\left(\alpha_{1}, \alpha_{2}, \cdots, \lambda_{i}, \cdots, \alpha_{n}\right) .
\end{gathered}
$$

Propriedade 4.13. Seja $n$ um inteiro tal que $n \geq 2$ e seja $M$ uma matriz qualquer de ordem $n$. Se uma fileira qualquer da matriz $M$ é uma combinação linear de outras fileiras paralelas, então $\operatorname{det}(M)=0$.

Para demonstrar essa propriedade, vamos supor primeiramente que a matriz $M$ é tal que $M=\left(\alpha_{1}, \cdots, \alpha_{i}, \cdots, \alpha_{p}, \cdots, \alpha_{j}, \cdots, \alpha_{n}\right)$. Sendo $\alpha_{j}=k \cdot \alpha_{i}+q \cdot \alpha_{p}$, dizemos que $\alpha_{j}$ é uma combinação linear de $\alpha_{i}$ e $\alpha_{p}$.

Como det é uma função $n$-linear das linhas de $M$, temos que

$$
\begin{gathered}
\operatorname{det}(M)=\operatorname{det}\left(\alpha_{1}, \cdots, \alpha_{i}, \cdots, \alpha_{p}, \cdots, \alpha_{j}, \cdots, \alpha_{n}\right) \\
\operatorname{det}(M)=\operatorname{det}\left(\alpha_{1}, \cdots, \alpha_{i}, \cdots, \alpha_{p}, \cdots, k \cdot \alpha_{i}+q \cdot \alpha_{p}, \cdots, \alpha_{n}\right) \\
\operatorname{det}(M)=\operatorname{det}\left(\alpha_{1}, \cdots, \alpha_{i}, \cdots, \alpha_{p}, \cdots, k \cdot \alpha_{i}, \cdots, \alpha_{n}\right)+\operatorname{det}\left(\alpha_{1}, \cdots, \alpha_{i}, \cdots, \alpha_{p}, \cdots, q \cdot\right. \\
\left.\alpha_{p}, \cdots, \alpha_{n}\right) \\
\operatorname{det}(M)=k \cdot \operatorname{det}\left(\alpha_{1}, \cdots, \alpha_{i}, \cdots, \alpha_{p}, \cdots, \alpha_{i}, \cdots, \alpha_{n}\right)+q \cdot \operatorname{det}\left(\alpha_{1}, \cdots, \alpha_{i}, \cdots, \alpha_{p}, \cdots,\right. \\
\left.\alpha_{p}, \cdots, \alpha_{n}\right)
\end{gathered}
$$

Como det é uma função alternada, $\operatorname{det}(A)=0$ sempre que a matriz $A$ tiver duas fileiras iguais, $\log$ temos que $\operatorname{det}(M)=k \cdot 0+q \cdot 0$. Com isso concluímos que

$$
\operatorname{det}(M)=0
$$

Propriedade 4.14. Seja $n$ um inteiro tal que $n \geq 2$ e seja $M$ uma matriz qualquer de ordem $n$. Se $M$ é uma matriz triangular, então $\operatorname{det}(M)$ é o produto dos elementos da diagonal principal da matriz $M$.

Para demonstrar essa propriedade, iremos inicialmente supor que $M$ é uma matriz triangular tal que $a_{i j}=0$ sempre que $i<j$, ou seja 


$$
M=\left(\begin{array}{ccccc}
a_{11} & 0 & 0 & \cdots & 0 \\
a_{21} & a_{22} & 0 & \cdots & 0 \\
a_{31} & a_{32} & a_{33} & \cdots & 0 \\
\vdots & \vdots & \vdots & \cdots & \vdots \\
a_{n 1} & a_{n 2} & a_{n 3} & \cdots & a_{n n}
\end{array}\right)
$$

Utilizando a Regra de Laplace na primeira linha, podemos calcular o determinante da matriz $M$ onde

$$
\operatorname{det}(M)=a_{11} \cdot \operatorname{det}\left(\begin{array}{cccc}
a_{22} & 0 & \cdots & 0 \\
a_{32} & a_{33} & \cdots & 0 \\
\vdots & \vdots & \cdots & \vdots \\
a_{n 2} & a_{n 3} & \cdots & a_{n n}
\end{array}\right)
$$

Aplicando novamente a Regra de Laplace na primeira linha da nova matriz encontrada temos

$$
\operatorname{det}(M)=a_{11} \cdot a_{22} \cdot \operatorname{det}\left(\begin{array}{ccc}
a_{33} & \cdots & 0 \\
\vdots & \cdots & \vdots \\
a_{n 3} & \cdots & a_{n n}
\end{array}\right)
$$

Repetindo o processo empregado nas $n-1$ matrizes encontradas a partir da primeira chegaremos a conclusão de que

$$
\operatorname{det}(M)=a_{11} \cdot a_{22} \cdot a_{33} \cdots a_{n n} .
$$

Cabe salientar que se $M$ é uma matriz triangular tal que $a_{i j}=0$ sempre que $i>j$, a demonstração decorre de forma análoga, porém escolhendo a última linha da matriz para aplicar a Regra de Laplace. 


\subsection{Abaixamento de ordem de um determinante (Re- gra de Chió)}

Veremos agora um processo baseado no Teorema de Jacobi que reduz a ordem de uma matriz da ordem $n$ para a ordem $n-1$ sem alterar o determinante dessa matriz.

Seja $n$ um inteiro tal que $n \geq 2$ e seja $A=\left(a_{i j}\right)$ uma matriz de ordem $n$ tal que $a_{11}=1$. Sendo $A$ representada abaixo, utilizaremos o seguinte processo:

$$
A=\left(\begin{array}{ccccc}
1 & a_{12} & a_{13} & \cdots & a_{1 n} \\
a_{21} & a_{22} & a_{23} & \cdots & a_{2 n} \\
a_{31} & a_{32} & a_{33} & \cdots & a_{3 n} \\
\vdots & \vdots & \vdots & \cdots & \vdots \\
a_{n 1} & a_{n 2} & a_{n 3} & \cdots & a_{n n}
\end{array}\right)
$$

1. Multiplicamos a primeira coluna por $-a_{12}$ e adicionamos o resultado à segunda coluna.

2. Multiplicamos a primeira coluna por $-a_{13}$ e adicionamos o resultado à terceira coluna.

3. Repetimos esse processo sempre multiplicando a primeira coluna por $-a_{1 j}$ e adicionamos o resultado a $j$-ésima coluna até que todas as $(n-1)$ colunas da matriz $A$ tenham seu primeiro elemento igual a zero.

Dessa forma, a matriz $A$ será transformada na matriz $B$ tal que

$$
B=\left(\begin{array}{ccccc}
1 & 0 & 0 & \cdots & 0 \\
a_{21} & a_{22}-a_{21} a_{12} & a_{23}-a_{21} a_{13} & \cdots & a_{2 n}-a_{21} a_{1 n} \\
a_{31} & a_{32}-a_{31} a_{12} & a_{33}-a_{31} a_{13} & \cdots & a_{3 n}-a_{31} a_{1 n} \\
\vdots & \vdots & \vdots & \cdots & \vdots \\
a_{n 1} & a_{n 2}-a_{n 1} a_{12} & a_{n 3}-a_{n 1} a_{13} & \cdots & a_{n n}-a_{n 1} a_{1 n}
\end{array}\right)
$$


Como todas as operações realizadas nas colunas da matriz $A$ seguiram o Teorema de Jacobi, concluímos que o determinante da matriz não foi alterado, logo $\operatorname{det}(A)=$ $\operatorname{det}(B)$.

Utilizando a Regra de Laplace na primeira linha da matriz $B$ a fim de calcular seu determinante, teremos

$$
\operatorname{det}(A)=\operatorname{det}(B)=\operatorname{det}\left(\begin{array}{cccc}
a_{22}-a_{21} a_{12} & a_{23}-a_{21} a_{13} & \cdots & a_{2 n}-a_{21} a_{1 n} \\
a_{32}-a_{31} a_{12} & a_{33}-a_{31} a_{13} & \cdots & a_{3 n}-a_{31} a_{1 n} \\
\vdots & \vdots & \cdots & \vdots \\
a_{n 2}-a_{n 1} a_{12} & a_{n 3}-a_{n 1} a_{13} & \cdots & a_{n n}-a_{n 1} a_{1 n}
\end{array}\right)
$$

Note que ao final desse processo, encontramos uma matriz de ordem $(n-1)$ que possui o mesmo determinante da matriz $A$, ou seja, abaixamos em uma unidade a ordem da matriz $A$ sem alterar o seu determinante.

Exemplo 4.7. Dada a matriz $A$ representada abaixo, vamos utilizar a Regra de Chió para rebaixar a ordem da matriz sem alterar seu determinante.

$$
A=\left(\begin{array}{cccc}
1 & 3 & 2 & -4 \\
2 & 7 & 1 & 6 \\
-4 & 5 & 3 & 4 \\
3 & 2 & 4 & -1
\end{array}\right)
$$

Utilizando a Regra de Chió, temos

$$
\left|\begin{array}{cccc}
1 & 3 & 2 & -4 \\
2 & 7 & 1 & 6 \\
-4 & 5 & 3 & 4 \\
3 & 2 & 4 & -1
\end{array}\right|=\left|\begin{array}{ccc}
7-6 & 1-4 & 6+8 \\
5+12 & 3+8 & 4-16 \\
2-9 & 4-6 & -1+12
\end{array}\right|=\left|\begin{array}{ccc}
1 & -3 & 14 \\
17 & 11 & -12 \\
-7 & -2 & 11
\end{array}\right|
$$

Como o elemento $b_{11}$ da nova matriz encontrada ainda é igual a 1 , podemos repetir o processo e reduzir mais uma unidade na ordem da matriz sem alterar seu determinante, logo teremos 


$$
\left|\begin{array}{ccc}
1 & -3 & 14 \\
17 & 11 & -12 \\
-7 & -2 & 11
\end{array}\right|=\left|\begin{array}{cc}
11+51 & -12-238 \\
-2-21 & 11+98
\end{array}\right|=\left|\begin{array}{cc}
62 & -250 \\
-23 & 109
\end{array}\right|
$$

Portanto, $\operatorname{det}(A)=62 \cdot 109-23 \cdot 250=1008$

\subsection{Regra de Crammer}

A seguir veremos uma generalização da resolução de sistemas lineares de $n$ equações e $n$ incógnitas utilizando o valor determinante.

Teorema 4.5.1. Seja $n$ um inteiro positivo. Seja um sistema linear de $n$ equações e $n$ incógnitas. Seja $x_{n}$ uma incógnita qualquer do sistema. Para todo $n$, podemos afirmar que $x_{n}($ det $)=\operatorname{det}_{x_{n}}$, onde det é o determinante da matriz formada por todos os coeficientes das incógnitas do sistema e onde $\operatorname{det}_{x_{n}}$ é o determinante dessa mesma matriz, trocando apenas a coluna dos coeficientes da incógnita $x_{n}$ pela coluna dos termos independentes.

Demonstração. Considere o sistema linear $A X=B$, onde

$$
A=\left(a_{i j}\right)_{n \times n}, X=\left(\begin{array}{c}
x_{1} \\
x_{2} \\
\vdots \\
x_{n}
\end{array}\right) \text { e } B=\left(\begin{array}{c}
b_{1} \\
b_{2} \\
\vdots \\
b_{n}
\end{array}\right)
$$

representam respectivamente as incógnitas e os termos independentes do sistema. Podemos representar esse sistema como

$$
S:\left\{\begin{array}{ccccccccccccc}
a_{11} x_{1} & + & a_{12} x_{2} & + & \cdots & + & a_{1 n-1} x_{n-1} & + & a_{1 n} x_{n} & = & b_{1} \\
a_{21} x_{1} & + & a_{22} x_{2} & + & \cdots & + & a_{2 n-1} x_{n-1} & + & a_{2 n} x_{n} & = & b_{2} \\
\vdots & + & \vdots & + & \cdots & + & \vdots & + & \vdots & = & \vdots \\
a_{n-11} x_{1} & + & a_{n-12} x_{2} & + & \cdots & + & a_{n-1 n-1} x_{n-1} & + & a_{n-1 n} x_{n} & = & b_{n-1} \\
a_{n 1} x_{1} & + & a_{n 2} x_{2} & + & \cdots & + & a_{n n-1} x_{n-1} & + & a_{n n} x_{n} & = & b_{n}
\end{array}\right.
$$


Considere a matriz obtida de $A$ ao substituirmos a primeira coluna de $A$ por $B$, ou seja a matriz

$$
\left(\begin{array}{cccc}
b_{1} & a_{12} & \cdots & a_{1 n} \\
b_{2} & a_{22} & \cdots & a_{2 n} \\
\vdots & \vdots & \cdots & \vdots \\
b_{n} & a_{n 2} & \cdots & a_{n n}
\end{array}\right)
$$

Substituindo cada $b_{i}$ por suas expressões dadas em $S$ e calculando o determinante, obtemos

$$
\left|\begin{array}{cccc}
a_{11} x_{1}+a_{12} x_{2}+\cdots+a_{1 n} x_{n} & a_{12} & \cdots & a_{1 n} \\
a_{21} x_{1}+a_{22} x_{2}+\cdots+a_{2 n} x_{n} & a_{22} & \cdots & a_{2 n} \\
\vdots & \vdots & \cdots & \vdots \\
a_{n-11} x_{1}+a_{n-12} x_{2}+\cdots+a_{n-1 n} x_{n} & a_{n-12} & \cdots & a_{n-1 n} \\
a_{n 1} x_{1}+a_{n 2} x_{2}+\cdots+a_{n n} x_{n} & a_{n 2} & \cdots & a_{n n}
\end{array}\right|
$$

Aplicando a propriedade da $n$-linearidade da função determinante podemos reescrever esse determinante como

$$
\left|\begin{array}{cccc}
a_{11} x_{1} & a_{12} & \cdots & a_{1 n} \\
a_{21} x_{1} & a_{22} & \cdots & a_{2 n} \\
\vdots & \vdots & \cdots & \vdots \\
a_{n 1} x_{1} & a_{n 2} & \cdots & a_{n n}
\end{array}\right|+\left|\begin{array}{cccc}
a_{12} x_{2} & a_{12} & \cdots & a_{1 n} \\
a_{22} x_{2} & a_{22} & \cdots & a_{2 n} \\
\vdots & \vdots & \cdots & \vdots \\
a_{n 2} x_{2} & a_{n 2} & \cdots & a_{n n}
\end{array}\right|+\cdots+\left|\begin{array}{cccc}
a_{1 n} x_{n} & a_{12} & \cdots & a_{1 n} \\
a_{2 n} x_{n} & a_{22} & \cdots & a_{2 n} \\
\vdots & \vdots & \cdots & \vdots \\
a_{n n} x_{n} & a_{n 2} & \cdots & a_{n n}
\end{array}\right|
$$

ou seja,

$$
x_{1}\left|\begin{array}{cccc}
a_{11} & a_{12} & \cdots & a_{1 n} \\
a_{21} & a_{22} & \cdots & a_{2 n} \\
\vdots & \vdots & \cdots & \vdots \\
a_{n 1} & a_{n 2} & \cdots & a_{n n}
\end{array}\right|+x_{2}\left|\begin{array}{cccc}
a_{12} & a_{12} & \cdots & a_{1 n} \\
a_{22} & a_{22} & \cdots & a_{2 n} \\
\vdots & \vdots & \cdots & \vdots \\
a_{n 2} & a_{n 2} & \cdots & a_{n n}
\end{array}\right|+\cdots+x_{n}\left|\begin{array}{cccc}
a_{1 n} & a_{12} & \cdots & a_{1 n} \\
a_{2 n} & a_{22} & \cdots & a_{2 n} \\
\vdots & \vdots & \cdots & \vdots \\
a_{n n} & a_{n 2} & \cdots & a_{n n}
\end{array}\right|
$$


Utilizando a propriedade que diz que uma função determinante é nula quando possui duas fileiras paralelas iguais temos que

$$
x_{1}\left|\begin{array}{cccc}
a_{11} & a_{12} & \cdots & a_{1 n} \\
a_{21} & a_{22} & \cdots & a_{2 n} \\
\vdots & \vdots & \cdots & \vdots \\
a_{n 1} & a_{n 2} & \cdots & a_{n n}
\end{array}\right|+x_{2} \cdot 0+x_{3} \cdot 0+\cdots+x_{n} \cdot 0
$$

A partir desse ponto podemos resumir o resultado encontrado da seguinte forma

$$
\left|\begin{array}{cccc}
b_{1} & a_{12} & \cdots & a_{1 n} \\
b_{2} & a_{22} & \cdots & a_{2 n} \\
\vdots & \vdots & \cdots & \vdots \\
b_{n} & a_{n 2} & \cdots & a_{n n}
\end{array}\right|=x_{1}\left|\begin{array}{cccc}
a_{11} & a_{12} & \cdots & a_{1 n} \\
a_{21} & a_{22} & \cdots & a_{2 n} \\
\vdots & \vdots & \cdots & \vdots \\
a_{n 1} & a_{n 2} & \cdots & a_{n n}
\end{array}\right|
$$

Denominando a matriz obtida trocando os coeficientes de $x_{1}$ pelos termos independentes como $\operatorname{det}_{x_{1}}$ temos $\operatorname{det}_{x_{1}}=x_{1}(\operatorname{det} A)$. Caso $\operatorname{det}(A) \neq 0$, concluímos que

$$
x_{1}=\frac{\operatorname{det}_{x_{1}}}{\operatorname{det}(A)}
$$

De forma análoga, se substituirmos na matriz $A$ os coeficientes da incógnita $x_{n}$ pelos termos independentes e depois de verificarmos que $\operatorname{det}(A) \neq 0$, concluiremos que

$$
x_{n}=\frac{\operatorname{det}_{x_{n}}}{\operatorname{det}(A)}
$$




\section{Capítulo 5}

\section{Atividades Propostas}

Neste capítulo propomos diversas atividades a cerca de todo o conteúdo abordado com o intuito de estabelecer uma linha de trabalho a ser aplicada em sala, com a qual o conteúdo poderá ser solidificado através da resolução dos exercícios propostos.

\subsection{Atividades sobre matrizes}

1. Represente explicitamente a matriz $A=\left(a_{i j}\right)$, com $1 \leq i \leq 2$ e $1 \leq j \leq 3$, tal que $a_{i j}=2 i+3 j-1$.

2. Seja $X=\left(x_{i j}\right)$ uma matriz quadrada de ordem 2 , onde

$$
\left\{\begin{array}{c}
i+j, \text { se } i=j \\
1-j, \text { se } i>j \\
1, \text { se } 1<j
\end{array}\right.
$$

Determine o valor da soma de seus elementos.

3. Determine os valores de $x$ e $y$ de modo que a equação matricial abaixo seja verdadeira.

$$
\left(\begin{array}{cc}
2 x & 3 y \\
3 & 4
\end{array}\right)=\left(\begin{array}{cc}
x+1 & 2 y \\
3 & y+4
\end{array}\right)
$$


4. Dadas as matrizes $A=\left(\begin{array}{ll}2 & 1 \\ 3 & 0\end{array}\right)$ e $B=\left(\begin{array}{cc}-1 & -3 \\ 4 & -2\end{array}\right)$ determine as matrizes $X$ e $Y$ tais que $\left\{\begin{array}{c}3 X+2 Y=5 A+B \\ X+3 Y=4 A-2 B\end{array}\right.$.

5. Obtenha os produtos $A B$ e $B A$ caso existam, sendo

$$
A=\left(\begin{array}{cc}
7 & -1 \\
4 & 3
\end{array}\right) \text { e } B=\left(\begin{array}{ccc}
-5 & 1 & -1 \\
2 & 3 & -2
\end{array}\right)
$$

6. Seja $A$ uma matriz de ordem 2 tal que $A=\left(\begin{array}{ll}1 & 1 \\ 0 & 1\end{array}\right)$.

a) Determine $A^{3}=A \cdot A \cdot A$

b) Se $A^{n}$ denota o produto de $A$ por $A, n$ vezes, determine o valor do número natural $k$ tal que $A^{k^{2}}-A^{5 k}+A^{6}=I$ onde $I$ é a matriz identidade.

7. Considere as matrizes $A$ e $B$ onde $B=\left(b_{i j}\right)$ é uma matriz de ordem $n \times p$ em que $b_{i j}=j^{i}$ e $A=\left(a_{i j}\right)$ é uma matriz quadrada de ordem $n$ tal que

$$
a_{i j}=\left\{\begin{array}{r}
1, \text { se } i \text { é par } \\
-1, \text { se } i \text { é impar }
\end{array}\right.
$$

a) Calcule a soma dos elementos da diagonal principal da matriz $A$

b) $\mathrm{O}$ elemento da quarta linha e da segunda coluna da matriz produto $A B$ é 4094. Calcule o número de linhas da matriz B.

8. Considere as matrizes $A=\left(\begin{array}{ccc}1 & 0 & -1 \\ 0 & -1 & 2\end{array}\right), I=\left(\begin{array}{ll}1 & 0 \\ 0 & 1\end{array}\right), X=\left(\begin{array}{l}x \\ y\end{array}\right) \mathrm{e}$ $B=\left(\begin{array}{l}1 \\ 2\end{array}\right)$. Se $x$ e $y$ são soluções do sistema $\left(A \cdot A^{t}-3 I\right) \cdot X=B$, determine o valor de $x+y$. 
9. Sendo $A$ a matriz representada abaixo, determine os valores de $x, y$ e $z$ para que $A$ seja uma matriz simétrica.

$$
A=\left(\begin{array}{ccc}
1 & x & 5 \\
2 & 7 & -4 \\
y & z & -3
\end{array}\right)
$$

10. Considere as matrizes

$A=\left(a_{i j}\right)_{4 \times 7}$, definida por $a_{i j}=i-j$

$B=\left(b_{i j}\right)_{7 \times 9}$, definida por $b_{i j}=i$

$C=c_{i j}$, tal que $C=A \cdot B$

Determine, caso exista, o elemento $c_{63}$.

11. Se $A=\left(\begin{array}{cc}x & y \\ 1 & 0\end{array}\right), B=\left(\begin{array}{ll}1 & z \\ 0 & z\end{array}\right)$ e $A B=B^{t}$ então determine $x+y+z$.

12. Se $A=\left(\begin{array}{ll}1 & 2 \\ 3 & 4\end{array}\right)$ e $B=\left(\begin{array}{ll}1 & 0 \\ 2 & 1\end{array}\right)$ então determine $M=(A+B)^{t}$

13. Se $A=\left(a_{i j}\right)$ é uma matriz quadrada de ordem 2 com $a_{i j}=2^{i}$ sempre que $i=j$ e $a_{i j}=0$ sempre que $i \neq j$, então determine $A^{-} 1$.

14. Sejam as matrizes $A=\left(a_{i j}\right)_{2 \times 2}$ em que $a_{i j}=2 i-3 j$ e $B=\left(b_{i j}\right)_{2 \times 2}$ em que $b_{i j}=\left\{\begin{array}{c}i-j, \text { se } i \leq j \\ i+j, \text { se } i>j\end{array}\right.$

Se $B^{t}$ é a matriz transposta de $B$, então determine $A-B^{t}$.

15. Determine a matriz $X$ de ordem 2 tal que $2 A+X=B$ sendo $A=\left(\begin{array}{cc}3 & 9 \\ 4 & -2\end{array}\right)$ e $B=\left(\begin{array}{cc}7 & 18 \\ 1 & -3\end{array}\right)$ 

$\begin{aligned} \text { 16. Se } A & =\left(\begin{array}{cc}\cos a & \sin a \\ -\sin a & \cos a\end{array}\right) \text { e } B=\left(\begin{array}{c}\cos 2 a \\ \sin 2 a\end{array}\right) \text {, determine a matriz } M \text { tal que } \\ A \cdot M & =B\end{aligned}$

17. Determine o número de matrizes $A=\left(a_{i j}\right)_{2 \times 2}$ onde $a_{i j}=\left\{\begin{array}{c}x, \text { se } i=j \\ y, \text { se } i \neq j\end{array}\right.$ tais que $A=A^{-1}$

18. Considere a matriz $A=\left(\begin{array}{ccc}2 & 5 & 1 \\ -1 & 4 & -3 \\ 3 & 0 & 2\end{array}\right)$. Efetuando-se $A^{t}-\frac{1}{3} A$ obtemos a $\operatorname{matriz}\left(\begin{array}{ccc}2 x+y & 5 x+y & \frac{8}{3} \\ \frac{16}{3} & \frac{8}{3} & 1 \\ 0 & -3 & \frac{4}{3}\end{array}\right)$. Calcule o valor absoluto de $\frac{y}{x}$

19. Sabendo-se que a matriz $\left(\begin{array}{ccc}2 & 1 & -1 \\ x^{2} & 0 & 1-y \\ x & y-3 & 1\end{array}\right)$ é simétrica, determine $x$ e $y$.

20. Dadas as matrizes $A=\left(\begin{array}{ll}2 & 1 \\ 1 & 1\end{array}\right)$ e $M=\left(\begin{array}{ll}1 & 0 \\ 2 & 1\end{array}\right)$

a) Determine $M^{-1}$

b) Determine a soma dos elementos da diagonal principal da matriz produto $M^{-1} \cdot A \cdot M$

21. Seja $A=\left(a_{i j}\right)_{2 \times 2}$ uma matriz onde $a_{i j}=\left\{\begin{array}{l}\cos j \pi, \text { se } i=j \\ \sin \frac{i \pi}{2}, \text { se } i \neq j\end{array}\right.$. Determine a matriz $A^{2}=A \cdot A$

22. Sejam as matrizes $A=\left(\begin{array}{ll}1 & 2 \\ 3 & 1\end{array}\right)$ e $M=\left(\begin{array}{ll}1 & 3 \\ 3 & 1\end{array}\right)$. Determine a matriz $X$ tal que $X=A^{-1} \cdot B$, onde $A^{-1}$ é a inversa da matriz $A$. 
23. Dada a equação matricial $\left(\begin{array}{l}9 \\ 3\end{array}\right)=\left(\begin{array}{cc}-2 & 1 \\ 1 & -2\end{array}\right) \cdot\left(\begin{array}{l}x \\ y\end{array}\right)$, Determine uma relação entre $x$ e $y$.

24. Sendo as matrizes $A=\left(\begin{array}{ccc}3 & 0 & 0 \\ 0 & -2 & 0 \\ 0 & 0 & 7\end{array}\right)$ e $B=\left(\begin{array}{ccc}5 & 0 & 0 \\ 0 & 2 & 0 \\ x & 0 & 7\end{array}\right)$, determine o valor de $x$ tal que $A B=B A$

25. Determine o valor de $x$ para que $\left(\begin{array}{c}2 n-1 \\ n-1\end{array}\right)=x \cdot\left(\begin{array}{c}2 n \\ n\end{array}\right)$

\section{$5.2 \quad$ Atividades sobre Determinantes}

1. Mostre que para qualquer matriz $A$ de ordem 2 ou de ordem $3, \operatorname{det}(A)=0$ sempre que $A$ tiver duas linhas ou duas colunas iguais.

Sugestão: Represente uma matriz $A$ qualquer por letras com duas fileiras iguais e calcule o determinante.

2. Mostre que para uma matriz $A$ qualquer de ordem 2 ou de ordem 3 , temos $\operatorname{det}(A)=-\operatorname{det}(B)$ sempre que $B$ for a matriz obtida permutando duas fileiras paralelas de $A$.

Sugestão: Represente uma matriz $A$ qualquer por letras, em seguida construa a matriz $B$ e calcule seus respectivos determinantes.

3. Mostre que para uma matriz $A$ qualquer de ordem 2 ou de ordem 3 , temos $\operatorname{det}(A)=\operatorname{det}\left(A^{t}\right)$ sempre que $A^{t}$ for a matriz transposta de $A$.

Sugestão: Represente uma matriz $A$ qualquer por letras, em seguida construa a matriz $A^{t}$ e calcule seus respectivos determinantes.

4. Sendo as matrizes $A=\left(a_{i j}\right)_{3 \times 2}$ onde $a_{i j}=2 j-i$ e também $B=\left(b_{i j}\right)_{2 \times 3}$ onde $b_{i j}=\left\{\begin{array}{c}0, \text { se } i=j \\ 2 i-j^{2}, \text { se } i \neq j\end{array}\right.$. Determine $\operatorname{det}(A B)$ 
5. Determine a solução da equação $\left|\begin{array}{ccc}2 & 1 & 3 \\ 4 & -1 & n-1 \\ n & 0 & n\end{array}\right|=12$

6. Determine uma solução geral para a equação $\left(\begin{array}{cc}\sin x & \cos x \\ \tan x & \cot x\end{array}\right)=0$

7. Se $a_{1}, a_{2}, \cdots, a_{9}$ formam nesta ordem uma progressão geométrica de razão $q$, então calcule o determinante da matriz $\left(\begin{array}{ccc}a_{1} & a_{2} & a_{3} \\ a_{4} & a_{5} & a_{6} \\ a_{7} & a_{8} & a_{9}\end{array}\right)$.

8. Calcule os determinantes das matrizes abaixo.
a) $\left|\begin{array}{ccccc}-1 & 0 & 0 & 0 & 0 \\ 2 & 4 & 0 & 0 & 0 \\ -1 & -2 & 1 & 0 & 0 \\ 2 & 4 & 3 & -2 & 0 \\ 1 & 0 & -1 & 3 & 2\end{array}\right|$
b) $\left|\begin{array}{cccc}1 & 4 & 3 & 1 \\ -1 & 2 & 3 & 1 \\ 0 & 1 & -1 & 2 \\ 0 & 0 & 2 & 3\end{array}\right|$
c) $\left|\begin{array}{cccc}1 & 1 & 1 & 1 \\ 2 & -3 & 5 & 4 \\ 4 & 9 & 25 & 16 \\ 8 & -27 & 125 & 64\end{array}\right|$ 
9. Seja $x$ a raiz da equação $\left|\begin{array}{cccc}x & 0 & 0 & 0 \\ 1 & x & 1 & 2 \\ 2 & 0 & x & 3 \\ 0 & 0 & 0 & 2\end{array}\right|=16$, calcule o valor de $x^{2}$.

10. Dada a matriz $A=\left(\begin{array}{ccccc}x & 1 & 0 & 0 & 0 \\ 0 & x & 1 & 0 & 0 \\ 0 & 0 & x & 1 & 0 \\ 0 & 0 & 0 & x & 8 \\ 0 & 0 & 1 & 0 & x\end{array}\right)$. Seja $f: R \rightarrow R$ uma função definida por $f(x)=\operatorname{det}(A)$. Calcule o valor de $\operatorname{det}(-1)$.

11. Calcule o valor de $\left|\begin{array}{ccccc}1 & 0 & 0 & 0 & 0 \\ 0 & 2 & 0 & 0 & 0 \\ 0 & 0 & 3 & 0 & 0 \\ 0 & 0 & 0 & 4 & 0 \\ 0 & 0 & 0 & 0 & 5\end{array}\right|+\left|\begin{array}{llllll}0 & 0 & 0 & 0 & 0 & 1 \\ 0 & 0 & 0 & 0 & 2 & 0 \\ 0 & 0 & 0 & 3 & 0 & 0 \\ 0 & 0 & 4 & 0 & 0 & 0 \\ 0 & 5 & 0 & 0 & 0 & 0 \\ 6 & 0 & 0 & 0 & 0 & 0\end{array}\right|$

12. Sabe-se que $M$ é uma matriz quadrada de ordem 3 e que $\operatorname{det}(M)=2$, então calcule $\operatorname{det}(3 M)$.

13. Resolva a equação $\left|\begin{array}{cccc}1 & 1 & 1 & 1 \\ x & -2 & 5 & -4 \\ x^{2} & 4 & 25 & 16 \\ x^{3} & -8 & 125 & -64\end{array}\right|=0$

14. Se $A=\left(\begin{array}{cc}\sin x & \cos x \\ -\cos x & \sin x\end{array}\right)$, calcule a matriz inversa de $A$. 
15. Calcule os valores de que satisfazem a equação $\left|\begin{array}{cccc}0 & 2 & 1 & 0 \\ 0 & 1 & 3 & k \\ 1 & 0 & k & 2 \\ 2 & 1 & 0 & 3\end{array}\right|=0$

16. Calcule o valor de $\left|\begin{array}{ccc}1 & 1 & 1 \\ \log 7 & \log 70 & \log 700 \\ (\log 7)^{2} & (\log 70)^{2} & (\log 700)^{2}\end{array}\right|$.

17. Calcule o valor de $\left|\begin{array}{cccc}1 & 1 & 1 & 1 \\ \log 8 & \log 80 & \log 800 & \log 8000 \\ (\log 8)^{2} & (\log 80)^{2} & (\log 800)^{2} & (\log 8000)^{2} \\ (\log 8)^{3} & (\log 80)^{3} & (\log 800)^{3} & (\log 8000)^{3}\end{array}\right|$

18. Se $X=\left|\begin{array}{ccc}8 & 7 & 4 \\ 10 & 1 & 5 \\ 0 & 20 & 1\end{array}\right|$ e $Y=\left|\begin{array}{ccc}8 & 7 & 4 \\ 0 & 20 & 1 \\ 10 & 1 & 5\end{array}\right|$, então estabeleça uma relação entre $X$ e $Y$.

19. Calcule o valor de $x$ na equação $\left|\begin{array}{ccc}3 & y & -1 \\ 2 & 1 & 4 \\ 1 & 0 & -2\end{array}\right|=\left|\begin{array}{cc}3 & x \\ -2 & -1\end{array}\right|$

20. Se $\left|\begin{array}{ccc}1 & 0 & 1 \\ 2 & 4 & 3 \\ x & y & 5\end{array}\right|=6$ e $\left|\begin{array}{ccc}3 & 1 & x \\ 2 & y & -1 \\ 0 & 3 & 5\end{array}\right|=47$, calcule o valor de $x+y$.

21. Seja $A=\left(a_{i j}\right)$ uma matriz quadrada de ordem 4. Calcule o valor $\operatorname{de} \operatorname{det}(A)$ 
sabendo que $a_{i j}=\left\{\begin{array}{c}0, \text { se } i<j \\ i+j, \text { se } i=j \\ i-j, \text { se } i>j\end{array}\right.$

22. Seja $A=\left(a_{i j}\right)$ uma matriz quadrada de ordem 2. Calcule o valor $\operatorname{de} \operatorname{det}(A)$ sabendo que $a_{i j}=2 i^{2}+j$

23. Seja $A=\left(a_{i j}\right)$ uma matriz quadrada de ordem 2. Calcule o valor $\operatorname{de} \operatorname{det}(A)$ sabendo que $a_{i j}=j-i^{2}$

24. Utilizando a Regra de Sarrus vista anteriormente, calcule os determinantes das matrizes $\left|\begin{array}{ccc}9 & 7 & 11 \\ -2 & 1 & 13 \\ 5 & 3 & 6\end{array}\right|,\left|\begin{array}{ccc}2 & -1 & 0 \\ m & n & 12 \\ 3 & 5 & 4\end{array}\right| \mathrm{e}\left|\begin{array}{ccc}0 & a & c \\ -c & 0 & b \\ a & b & 0\end{array}\right|$

25. Calcule o valor de $D=\left|\begin{array}{ccc}2 & \log _{5} 5 & \log _{5} 5 \\ 5 & \log _{5} 125 & \log _{5} 25 \\ 8 & \log _{3} 27 & \log _{3} 243\end{array}\right|$

26. Se somarmos 4 a todos os elementos da matriz $A$ cujo determinante é $D$ obteremos a matriz $B$. Calcule o determinante da matriz $B$ sendo $A=\left|\begin{array}{ccc}1 & 2 & 3 \\ 1 & 1 & m \\ 1 & 1 & 1\end{array}\right|$

27. Calcule o determinante da matriz $A=\left(\begin{array}{ccc}\sin ^{2} x & \sin ^{2} x & 0 \\ \cos ^{2} x & \cos ^{2} y & \sin ^{2} y \\ r^{2} & 0 & r^{2}\end{array}\right)$

28. Chama-se traço de uma matriz quadrada ao valor da soma dos elementos da diagonal principal dessa matriz. Sabendo que o traço da matriz abaixo é igual a 9 e seu determinante igual a 15 , calcule os elementos $x$ e $y$ dessa matriz. 


$$
\left(\begin{array}{lll}
1 & 2 & 3 \\
0 & x & z \\
0 & 0 & y
\end{array}\right)
$$

29. Calcule o cofator do elemento 3 na matriz $M=\left(\begin{array}{cccc}2 & 4 & 1 & 0 \\ 6 & -2 & 5 & 7 \\ -1 & 7 & 2 & 4 \\ 0 & 3 & -1 & -10\end{array}\right)$

30. Sendo $D_{i j}$ e $A_{i j}$ respectivamente o menor complementar e o cofator da matriz

$$
A=\left(a_{i j}\right) \text { de ordem } n \text {, calcule } D_{13} \text { e } A_{24} \text { da matriz } M=\left(\begin{array}{cccc}
1 & -1 & 0 & 0 \\
0 & 2 & -2 & 1 \\
3 & 3 & 4 & 1 \\
4 & 5 & 7 & 6
\end{array}\right)
$$

31. Calcule os determinantes das matrizes abaixo.
a) $M=\left(\begin{array}{cccc}1 & 0 & -1 & 3 \\ 2 & 3 & 4 & 2 \\ 0 & 2 & 5 & 1 \\ 4 & 1 & 0 & 0\end{array}\right)$
b) $N=\left(\begin{array}{llll}2 & 4 & 2 & 4 \\ 0 & 1 & 1 & 0 \\ 1 & 0 & 2 & 3 \\ 3 & 0 & 1 & 0\end{array}\right)$ 
c) $P=\left(\begin{array}{ccccc}1 & 2 & 3 & -4 & 2 \\ 0 & 1 & 0 & 0 & 0 \\ 0 & 4 & 0 & 2 & 1 \\ 0 & -5 & 5 & 1 & 4 \\ 0 & 1 & 0 & -1 & 2\end{array}\right)$

32. Calcule os determinantes abaixo usando propriedades.
a) $\left(\begin{array}{ccc}x^{2} & x y^{2} & x \\ x y & y^{3} & y \\ x^{2} & y^{2} & x\end{array}\right)$
b) $\left(\begin{array}{ccccc}3 & 5 & 0 & 4 & 7 \\ 2 & 13 & 0 & 19 & 17 \\ 9 & 27 & 0 & 25 & 35 \\ 16 & 51 & 0 & 42 & 47 \\ 21 & 73 & 0 & 54 & 49\end{array}\right)$

33. Sabendo que $A$ é uma matriz quadrada de ordem 4 e que $\operatorname{det}(A)=-6$, calcule o valor de $x$ tal que $\operatorname{det}(2 A)=x-97$.

34. Sem desenvolver os determinantes, prove que $D^{\prime}=8 D$ sendo

$$
D=\left|\begin{array}{cccc}
x & x^{2} & x^{3} & x^{4} \\
y & y^{2} & y^{3} & y^{4} \\
z & z^{2} & z^{3} & z^{4} \\
t & t^{2} & t^{3} & t^{4}
\end{array}\right| \text { e } D^{\prime}=\left|\begin{array}{cccc}
8 x & -2 x^{2} & 2 x^{3} & -2 x^{4} \\
4 y & -y^{2} & y^{3} & -y^{4} \\
4 z & -z^{2} & z^{3} & -z^{4} \\
4 t & -t^{2} & t^{3} & -t^{4}
\end{array}\right|
$$

35. Verifique a identidade seguinte aplicando as propriedades dos determinantes. 


$$
\left|\begin{array}{ccc}
\cos 2 a & \cos ^{2} a & \sin ^{2} a \\
\cos 2 b & \cos ^{2} b & \sin ^{2} b \\
\cos 2 c & \cos ^{2} c & \sin ^{2} c
\end{array}\right|=0
$$

36. Demonstre sem desenvolver o determinante que

$$
\left|\begin{array}{ccc}
a-b & m-n & x-y \\
b-c & n-p & y-z \\
c-a & p-m & z-x
\end{array}\right|=0
$$

37. Mostre que $(a+b+c)$ é um fator de $\left|\begin{array}{ccc}(b+c)^{2} & b^{2} & c^{2} \\ a^{2} & (a+c)^{2} & c^{2} \\ a^{2} & b^{2} & (a+b)^{2}\end{array}\right|$

38. Demonstre sem desenvolver o determinante que

$$
\left|\begin{array}{ccc}
\cos 0 & \cos a & \cos 2 a \\
\cos a & \cos 2 a & \cos 3 a \\
\cos 2 a & \cos 3 a & \cos 4 a
\end{array}\right|=0
$$

39. Prove que $\left|\begin{array}{ccc}a^{2} & (a+2)^{2} & (a+4)^{2} \\ (a+2)^{2} & (a+4)^{2} & (a+6)^{2} \\ (a+4)^{2} & (a+6)^{2} & (a+8)^{2}\end{array}\right|=-2^{9}$ 


\section{Considerações Finais}

De forma ampla, a matemática em sua grande parte, se dedica a responder perguntas que na maioria das vezes são expressas por equações. Dentre as várias formas de resolver essas equações, uma foi estudada e algoritmizada e um dos passos desse procedimento se destacou como sendo o estudo dos determinantes.

Com o passar do tempo, o determinante foi tomando uma importância cada vez maior dentro do estudo matemático até que começou a ser visto não só como uma ferramenta para solucionar equações, mas como um tópico dedicado de estudo que se ramifica em várias partes e com enumeras aplicações. Atualmente o determinante é estudado inicialmente no segundo ano do Ensino Médio e tanto seu significado quanto sua aplicação não são levados em conta no processo de Ensino e Aprendizagem. Vimos em uma abordagem básica, a origem do determinante que na opinião da maioria dos historiadores teve sua mais remota apreciação na china antiga, datando provavelmente de 300 anos antes de Cristo. Mostramos a existência de uma função determinante e após algumas conceituações e demonstração, chegamos a conclusão de que além de existir, essa função determinante é única. Conceituamos e demonstramos a veracidade de diversas propriedades aplicadas ao determinante e como essas propriedades são úteis para o cálculo do determinante em diversos casos. Deixamos ainda uma série de atividades propostas para aplicação no Ensino Médio que percorrem todos os tópicos abordados neste trabalho.

Na parte denominada Reconstrução do Determinante, existe uma proposta clara de trabalho a ser aplicada no Ensino Médio a fim de tirar o conceito abstrato e mecanizado do determinante que em geral é passado nos livros didáticos e trazê-lo a uma luz mais concreta, mostrando ao aluno o que é esse determinante de forma prática na resolução de sistemas lineares. Com a aplicação da proposta, espera-se que além de praticar o método de resolução de sistemas de $2^{\mathrm{a}}$ e de $3^{\mathrm{a}}$ ordem, o aluno possa gradualmente 
entender a ideia fundamental do estudo proposto e mesmo que venha a algoritmizar o processo a posterior, que esse aluno saiba de onde o processo se originou. Aborda-se ainda na proposta uma interpretação geométrica dos sistemas de equações de segunda e terceira ordem em cada caso a partir dos valores encontrados para a função determinante o que acreditamos vir a concretizar mais ainda o significado do determinante e sua aplicação na resolução desses sistemas específicos. 


\section{Referências Bibliográficas}

[1] Iezzi, G e Hazzan, S, Fundamentos de Matemática Elementar, Atual, vol. 4 (1993).

[2] Hoffman, K e Kunze, R, Álgebra Linear, Poligono, (1971).

[3] BOyer, C.B., História da Matemática, Edgard Blucher, (1996).

[4] Paiva, M., Matemática, Moderna, Vol. 2 (2013).

[5] Dante, L.R., Matemática, Contextos e Aplicações, Ática, Vol. 2 (2012).

[6] Iezzi, G; Dolce, O; Degenszajn, D e PéRigo, R, Matemática, Atual, vol. Único (2013).

[7] Youssef, A.n; Soares, E; e Fernandes, V.P., Matemática, Scipione, vol. Único (2008).

[8] Silva, C.X. E Filho, B.B., Matemática, FTD, vol. 2 (2005). 